Distribution Category: Atomic, Molecular, and Chemical Physics

(UC-411)

ANL- $-92 / 20$

ANL-92/20

DE93 007435

ARGONNE NATIONAL LABORATORY

9700 South Cass Avenue

Argonne, nlinois 60439

\title{
7-GeV Advanced Photon Source Instrumentation Initiative. Conceptual Design Report
}

December 1992

work sponsored by U.S. DEPARTMENT OF ENERGY

Office of Energy Research 


\section{TABLE OF CONTENTS}

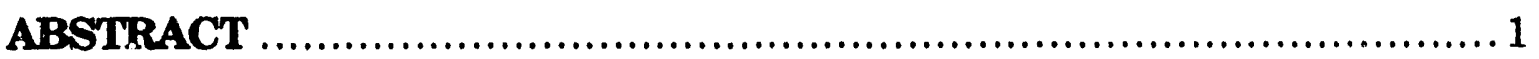

\section{CHAPTER I BACKGROUND AND OVERVIEW}

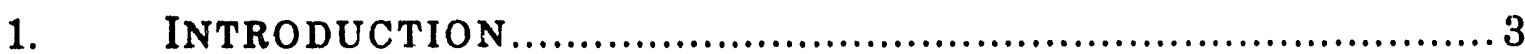

$1.1 \quad$ Background........................................................... 3

1.2 Scope of the APS Instrumentation Initiative......................... 4

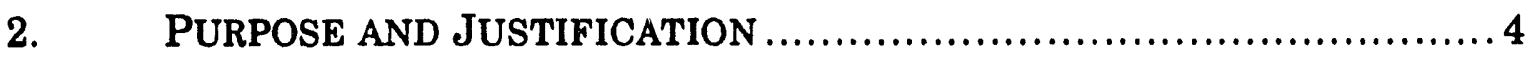

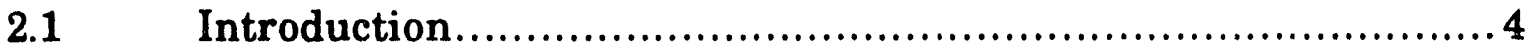

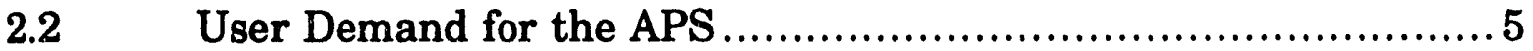

2.3 Beamline Plan in the Instrumentation Initiative .................5

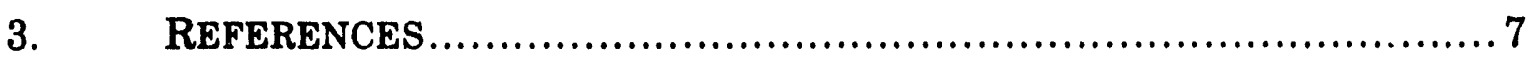

\section{CHAPTER II EXPERIMENTAL FACILITIES}

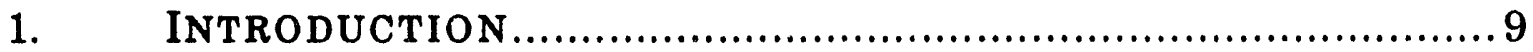

2. GENERAL LAYOUT OF THE EXPERIMENTAL FACILITIES ..............10

$2.1 \quad$ Introduction......................................................... 10

2.2 Disposition of Instrumentation Initiative Beamlines................10

3. INSERTION DEVICES.......................................................12

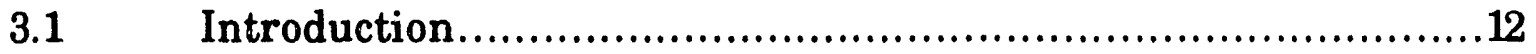

3.2 Insertion Devices in Type A Sectors..................................14

3.2.1 Undulators ...................................................... 14

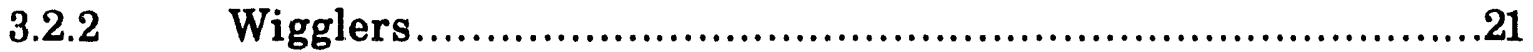

3.2.3 Insertion Device Vacuum Chambers for Type A Sectors..........21

3.3 Insertion Devices in Type B Sectors ...............................24

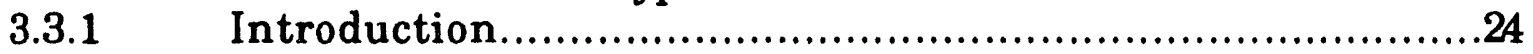

3.3.2 In-Vacuum Undulators ......................................24

3.3.3 Circular Polarization Sources ....................................26

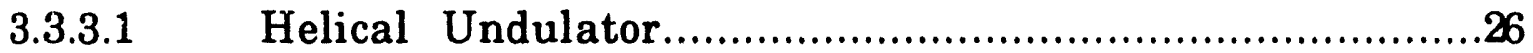

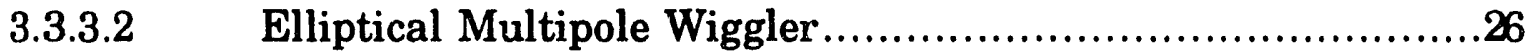

3.3.4 Wigglers with Large Critical Energies ...........................30

3.3.5 Double Undulator Sources on a Single Straight Section ..........36

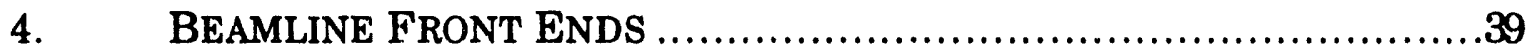

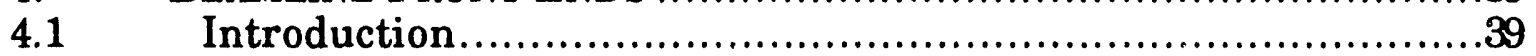

4.2 Front Ends in Type A and Type B Sectors ..........................41 
5. BEAMLINES IN THE APS INSTRUMENTATION INITIATIVE ...........44

$5.1 \quad$ Introduction....................................................44

5.2 Description of Typical Special Purpose Beamlines...................44

5.2.1 Three-Dimensional Holographic Imaging Facility ..............44

5.2.2 Very High Energy X-ray Scattering Facility ....................46

5.2.3 Complete Polarization Facility..........................................50

5.2.4 Sub-Nanosecond Temporal Resolution Beamline Facility ........53

5.2.5 Facility for Coherence and Interference Techniques .............56

5.2.6 Static and Dynamic Small Angle X-ray Scattering Facility ......60

5.2.7 Ultra-High-Pressure and Ultra-High-Temperature Facility ...63

5.2.8 Kilometer Beamline for X-ray Imaging ...........................64

5.2.9 Chemical and Structural Kinetics Facility ...........................67

5.2.10 Biotechnology Development Facility ...................................68

5.2.11 Development of X-ray Fabrication Process ..........................70

5.2.12 X-ray Manufacturing and Engineering Research Facility .......71

6. DATA HANDLING AND COMMUNICATIONS.................................73

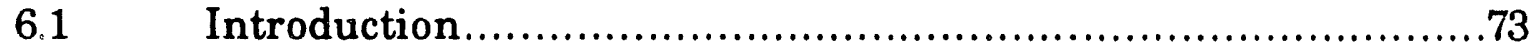

$6.2 \quad$ Handling High Data Rates .............................................73

6.3 APS Data Handling Infrastructure Improvements................75

$6.4 \quad$ Visualization Tools .....................................................75

6.5 Control and Monitoring ...........................................

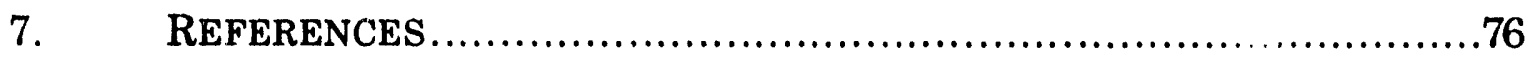

\section{CHAPTER III USER FACIITY BUILDINGS}

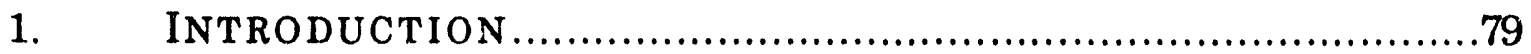

2. LABORATORY-OFFICE MODUlES........................................79

$2.1 \quad$ Architecture............................................................8 80

2.2 Operational and Functional Needs.........................................80

$2.3 \quad$ Structural Systems..............................................................8 80

2.4 Heating, Ventilating, and Air Conditioning .........................82

2.5 Plumbing, Process Piping, and Fire Protection......................82

$2.6 \quad$ Electrical Power...........................................................82

3. X-RAY IMAGING LABORATORY ........................................8 83

3.1 Introduction ............................................................ 83

3.2 Architecture................................................................83

$3.3 \quad$ Operational and Functional Needs........................................86

3.4t Structural Systems.................................................. 86

3.5 Heating, Ventilating, and Air Conditioning .....................87

3.6 Plumbing, Process Piping, and Fire Protection........................87

Electrical Power...........................................................87 


\section{CHAPTER IV COST AND SCHEDULE}

1. INTRODUCTION TO COST AND SCHEDULE..................................89

2. COST ESTIMATES..........................................................

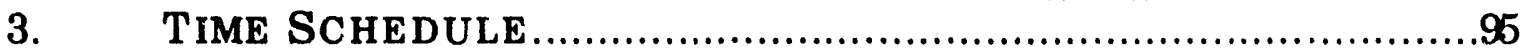

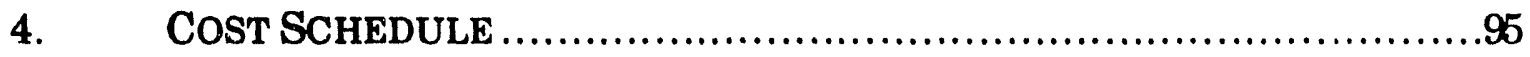

APPENDIX A PRECONSTRUCTION R\&D PROGRAM .................. 105

APPENDIX B COPY OF SCHEDULE 44 ..................................... 109 


\section{LIST OF FIGURES}

2.2.1 Disposition of sectors at the APS. In the new initiative, Sectors 9 through 12 will be Type A Sectors. The Type B Sectors will be Sector 21 through Sectors 26

2.3.1 A comparison of the on-axis brilliance for the APS bending magnet source, Wiggler $\mathrm{A}$, and Undulator $\mathrm{A}$ 16

2.3.2- Energy spectra of radiation from Undulator A for three values

2.3.4 of $\mathrm{K}$. The positron beam parameters in these calculations are: horizontal beam width $\sigma_{\mathbf{x}}^{\prime}: 308 \mu \mathrm{m}$, vertical beam height $\sigma_{y}^{\prime}: 85 \mu \mathrm{m}$, horizontal beam divergence $\sigma_{x}^{\prime}: 24 \mu \mathrm{rad}$, vertical beam divergence $\sigma^{\prime} \mathrm{y}: 9 \mu \mathrm{rad}$ 18

2.3.5 Optimized 2D magnetic flux profile of a quarter period of Undulator $A$

2.3.6 Cross section of the ID vacuum chamber

2.3.7 A possible magnet design for a helical undulator with a planar geometry and made of rectangular pieces of permanent magnet. The arrow shown corresponds to the orientation of magnetization (Ref. 5)

2.3.8 Placement of magnetic field components to generate circularly polarized radiation from the elliptical motion of positrons in a wiggler. The $\mathrm{B}_{\mathrm{X}}$ and $\mathrm{B}_{\mathrm{y}}$ components have a special phase difference of $\pi / 2$ along the $\mathrm{z}$ axis. In this case, the on-axis radiation is right circularly polarized (Ref. 7)

2.3.9 Conceptual design for the elliptical multipole wiggler

2.3.10 Variation of $\mathrm{P}_{\mathrm{c}}$ as a function of photon energy for the elliptical multipole wiggler with a period of $15 \mathrm{~cm}$ operated at $7 \mathrm{GeV}$ for $K_{y}=15$ and various values of $K_{x}$

2.3.11 On-axis relative intensity of the circularly polarized beam as a function of photon energy for various values of $K_{\mathbf{X}}$

2.3.12 A conceptual layout of a superconducting wiggler providing a "single wiggle" of $7.7 \mathrm{~mm}$ radius by a $7-\mathrm{cm}$-long central coil with a field of $3 \mathrm{~T}$. The superconducting coils at either end are roughly $14 \mathrm{~cm}$ long and provide a field of $0.75 \mathrm{~T}$ 


\section{LIST OF FIGURES continued}

2.3.13 Brilliance as a function of photon energy for the permanent magnet wiggler $\left(E_{c}=58\right)$ and the superconducting wiggler $\left(E_{c}=98\right) \ldots .35$

2.3.14 On-axis brilliance from a 20-cm-period, 5-m-long, Nd-Fe-B hybrid device at two values of $\mathrm{K}$ proposed for the APS

2.3.15 Coherent flux from a 5-m-long undulator on the APS with a typical brilliance of $10^{18}$ photons $/ \mathrm{sec} / 0.1 \% \mathrm{BW} / \mathrm{mrad}^{2} / \mathrm{mm}^{2}$ over the energy range

2.4.1 Layout of the components on an insertion device beamline

front end

2.5.1 Principle of $\mathrm{x}$-ray holographic imaging at the APS 46

2.5.2 Conceptual design for Fresnel-type holography using two undulator sources

2.5.3 Total photon cross section $\sigma_{\text {tot }}$ in carbon, as a function of energy, showing the contributions of different processes: $\tau$ : atomic photoeffect (electron ejection, photon absorption); $\sigma_{c .0 h}$ : coherent scattering (Rayleigh scattering atom neither ionized nor excited); $\sigma_{\text {incoh}}$ incoherent scattering (Compton scattering off an electron); $\kappa_{\mathrm{n}}$ : pair production, nuclear field; $\kappa_{\mathrm{e}}$ : pair production, electron field; $\sigma_{\text {ph }}$ : photonuclear absorption (nuclear absorption, usually followed by emission of a neutron or other particle)

2.5.4 Conceptual layout of a beamline to perform elastic and inelastic scattering using a high-critical-energy wiggler source 51

2.5.5 Flux from a 7.5 Tesla superconducting wiggler on the APS as a function of energy. Comparison is made with a 1.8 Tesla permanent magnet wiggler and a 4 Tesla superconducting wiggler planned for ESRF

2.5.6 Schematic layout of a beamline for polarization studies using an elliptical multipole wiggler source on the APS 54

2.5.7 Schematic layout of beamline requirements for a high temporal resolution pump-probe beamline for the APS 


\section{LIST OF FIGURES continued}

2.5.8 Conceptual layout of an APS Instrumentation Initiative beamline for performing temporal studies using two undulators as radiation sources ...................................................... 58

2.5.9 Layout of a beamline for performing coherence and interference studies in the APS Instrumentation Initiative

2.5.10 Schematic layout showing the requirements of a beamline for the APS Instrumentation Initiative to perform small angle $x$-ray scattering studies

2.5.11 Schematic layout of an ultra-high-pressure and ultra-hightemperature beamline

2.5.12 Requirements of a kilometer-long beamline for $x$-ray imaging.........67

2.5.13 Beamline requirements to investigate chemical and structural kinetics

2.5.14 Principle of beamline for x-ray microfabrication ..................... 71

2.5.15 Layout of the beamline for x-ray manufacturing and engineering research

3.2.1 Layout of the APS facilities showing the location of the Laboratory-Office Modules in the current construction phase and the Instrumentation Initiative.

3.3.1 Location of the X-ray Imaging Laboratory on an ID source. The beamline extends from the perimeter of the Experiment Hall in a north-westerly direction

3.3.2 Details of the optics building and the detector building associated with the X-ray Imaging Laboratory 85

4.3.1 APS Instrumentation Initiative Construction Time Schedule...........96

4.4.1 APS Instrumentation Initiative Cost Schedule (FY 92 M\$) ............98

4.4.2 APS Instrumentation Initiative Cost Schedule .........................101

4.4.3 APS Instrumentation Initiative Budget Profile ........................103 


\section{LIST OF TABLES}

1.2.1 Status of Collaborative Access Teams (CATs) in the Current Phase (December 1992) ..........................................................6

2.3.1 Summary of Insertion Devices for Type A Sectors ..................... 17

2.3.2 Optimized Design Parameters of Nd-Fe-B Hybrid

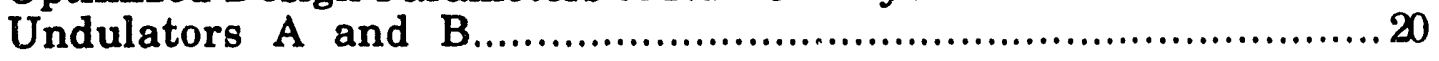

2.3.3 Partial List of Specifications for Undulators A and B .................. 20

2.3.4 Design Parameters for Wigglers A and B............................23

2.3.5 Parameters for Wigglers Designed for the APS Instrumentation Initiative .............................................. 33

2.4.1 Power and Power Densities Delivered by Various Insertions Devices in Type A Sectors

2.4.2 Power and Power Densities Delivered by Various

Insertion Devices in Type B Sectors ....................................... 43

2.5.1 Temporal Properties of Synchrotron Sources .......................... 55

4.1.1 APS Instrumentation Initiative Work Breakdown Structure ...........90

4.2.1 Cost Account Planning - Direct Cost Estimates......................... 92

4.2.2 Craft Codes and Hourly Rates (FY 92\$) .............................. 93

4.2.3 APS Instrumentation Initiative Cost Breakdown by WBS Code........99

4.4.1 APS Instrumentation Initiative Cost Schedule (FY $92 \mathrm{M} \$$ ) ............ 97

4.4.2 Escalated Rates.......................................................99

4.4.3 APS Instrumentation Initiative Cost Schedule (Escalated M\$)........100

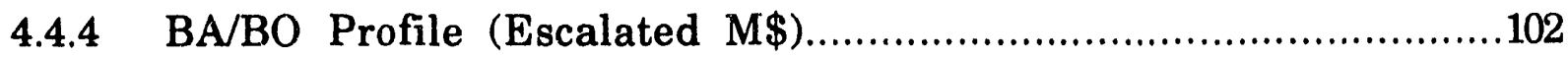




\title{
7-GeV AdVANCED PHOTON SOURCE APS INSTRUMENTATION INITIATIVE CONCEPTUAL DESIGN REPORT
}

\begin{abstract}
The DOE is building a new generation 6-7 GeV Synchrotron Radiation Source known as the Advanced Photon Source (APS) at Argonne National Laboratory (Project No. 89-R-402). This facility, to be completed in FY 1996, will provide 70 $\mathrm{x}$-ray sources of unprecedented brightness to meet the research needs of virtually all scientific disciplines and numerous technologies. The technological research capability of the APS in the areas of energy, communications, and health will enable a new partnership between the DOE and U.S. industry. Current funding for the APS will complete the current phase of construction so that scientists can begin their applications in FY 1996. It is now appropriate to plan to construct a set of specialized beamlines whose design will advance the state of the art in x-ray science. Comprehensive utilization of the unique properties of APS beams will enable cutting-edge research not currently possible. These new beamline facilities will be targeted for the most important science/technology goals of the U.S. x-ray community and will be available on a peer-review or proprietary basis to all researchers from universities, federal laboratories, and industry. Furthermore, a number of additional beamlines will be made available for development by Collaborative Access Teams.

In this APS Instrumentation Initiative, 2.5-m-long and 5-m-long insertion-device $x$-ray sources will be built on 9 straight sections of the APS storage ring, and an additional 9 bending-magnet sources will also be put in use. The front ends for these $18 x$-ray sources will be built to contain and safeguard access to these bright $x$-ray beams. In addition, funds will be provided to build state-of-the-art insertion-device beamlines to meet scientific and technological research demands well into the next century. This new initiative will also include four user laboratory modules and a special laboratory designed to meet the x-ray imaging research needs of the users.
\end{abstract}

The Conceptual Design Report (CDR) for the APS Instrumentation Intiative describes the scope of all the above technical and conventional construction and provides a detailed cost and schedule for these activities. According to these plans, this new initiative begins in FY 1994 and ends in FY 1998.

The document also describes the preconstruction R\&D plans for the Instrumentation Intiative activities and provides the cost estimates for the required $R \& D$. 


\section{CHAPTER I BACKGROUND AND OVERVIEW}

\section{INTRODUCTION}

\subsection{Background}

The 6-7 GeV Synchrotron Radiation Source for the Advanced Photon Source (APS) is now under construction at Argonne National Laboratory (Project No. 89$\mathrm{R}-402)$. This is a synchrotron radiation facility with the unique capability to produce highly brilliant x-ray beams. 1 User groups are now forming to make use of the x-rays from the APS in a variety of research endeavors, from basic science to industrial technology. These groups cover a very wide range of disciplines from condensed matter physics and materials sciences to chemistry and biology, from energy research to pharmaceutical research, from medical research to health sciences research, and from earth sciences to environmental sciences.

The APS facility was planned based on the recommendations by the Eisenberger-Knotek Committee, 2 which were later studied and endorsed by the Major Materials Facilities Committee of the National Academy of Sciences, chaired by Fredric Seitz and Dean Eastman. 3 The highest priority need for this facility was strongly endorsed by the National Research Council (Brinkman) Committee ${ }^{4}$ and by two subcommittees of the DOE Energy Research Advisory Board . 5,6

Recently, the SEAB Task Force on Energy Research 7 appointed by the Energy Secretary reiterated the above through the following statement: "The Task Force supports the continuation, on the current baseline of cost and schedule, of the Advanced Photon Source (APS) [current phase]. The APS was the highest priority of the 1984 National Academy of Sciences study on major materials facilities, and the Task Force believes that the construction of this thirdgeneration synchrotron facility remains a very high scientific priority for the Department." The DOE's commitment to the construction of the APS is very evident from the funding of its construction, which began in FY 1989.

The current construction phase of the APS will be completed in FY 1996 when the user research programs will begin. However in the current phase of this project, only 32 of the 69 possible $x$-ray sources will be ready for use. Within the scope of current phase construction, insertion devices and beamline front ends will be built and installed by the APS so that the users can build 32 beamlines on the experiment floor to perform research. The $32 \mathrm{x}$-ray sources to be completed in the current phase have already been committed to their full use through this century by the scientific and technological users. There are many more users, who could not participate in the current phase, already committing to use the $x$ ray sources proposed in this Instrumentation Initiative when they become available. Hence, it is necessary to plan this new initiative to construct the necessary equipment to make the additional $x$-ray sources functional for user 
programs. The effective use of the APS will be optimal only when the new construction proposed in this Conceptual Design Report is completed.

In this regard, it is useful to recall the guidance 8 from the Energy Secretary for planning of 1994 budget: "...High priority must be given to maintaining, staffing, and effectively using these [SSC and APS] and existing facilities before considering additional projects..."

In light of the above facts, it is timely to present the APS Instrumentation Initiative, which proposes the construction to begin in FY 1994 and be completed in FY 1998. The 7-GeV Advanced Photon Source Instrumentation Initiative Conceptual Design Report (CDR) presents various elements of this construction project, including the costs and schedules.

\subsection{Scope of the APS Instrumentation Initiative}

In the APS Instrumentation Initiative, 2.5-m-long insertion device $x$-ray sources and specialized insertion cevire $x$-ray sources with unique capabilities will be built on an additional 9 st $\mathrm{s}^{\text {hh }}$ t sections of the APS storage ring. Also, an additional 9 bending magnet sources will be put to use. The front ends for these 18 $x$-ray sources will be built to contain and safeguard access to these bright x-ray beams. In addition, partial funds will be provided to build state-of-the-art insertion device beamlines on the experiment floor to meet the scientific and technological research demands of the next century.

The APS Instrumentation Initiative also proposes to build four laboratoryoffice modules for the users. These modules are similar to the four included in the current construction project. They contain the first-floor area composed of office space, chemistry labs, electronics labs, toilet facilities, truck-dock air lock, compressed-gas storage, reception area, and work-break area. In addition, a specialized laboratory will be added to meet specific scientific goals of the users performing research on $x$-ray imaging.

\section{Purpose and Justification}

\subsection{Introduction}

\section{Purpose, Justification of Need for, and Scope of Project}

The Advanced Photon Source is in great demand by scientific and technological researchers from the fields of physics, materials, chemistry, biology, energy, pharmaceuticals, and medicine. The new Instrumentation Initiative has two main goals: first, to provide new opportunities to the technological and academic users in areas not possible with current instrumentation technology; second, to support the unexpected demand for bright $x$-ray beams by the user communitythat could not be met in the funded phase of the project. 
The APS Instrumentation Initiative reflects the need for the ultra-bright $x$-ray beams by industrial and academic users. Applications continue to increase, and they span a wide variety of scientifir disciplines, for example, from studies of biological cells to structure of drugs iv non-evasive diagnostics of the human heart, from physical sciences to environmental sciences. In order to effectively exploit this national scientific resource, the complement of instrumentation and laboratory space proposed here to support users must be provided.

The importance of this facility for industrial competitiveness of the U.S. is clear. The European countries are jointly building a facility in Grenoble, France, which is similar to the 6-7 GeV Synchrotron Radiation Source being built at Argonne. The Japanese are building a facility in Nishi-Harima that is larger than the APS Project even after the new Instrumentation Initiative is completed.

The demand for the APS Instrumentation Initiative reflects unusual research needs for the ultra-bright $x$-ray beams by industrial and academic users. The applications continue to increase in number with the opening of many unanticipated directions in research. Thus, for example, the capability to obtain three-dimensional holograms of a molecular structure, which has been considered very difficult if not impossible, will be a reality with the techniques to be 'eveloped in the new initiative.

\subsection{User Demand for the APS}

In Table 1.2.1, the status (as of December 1992) of the Collaborative Access Teams (CATs) formed by the APS users is given. The number of CATs approved by the APS Proposal Evaluation Bn $r$ rd (PEB) is 15. These 15 CATs are in need of 19 APS sectors to build 38 beamlinus. On the other hand, the APS will develop only 16 sectors where users group can build 32 beamlines. At this time, the additional requests for 6 beamlines by the APS user groups can, hence, only be accommodated in the new initiative project. Experience indicates that this demand will only grow during the soming years.

\subsection{Beamline Plan in the Instrumentation Initiative}

In the APS Instrumentation Initiative, the plan is to prepare an additional 18 beamlines occupying nine sectors of the APS. There will be nine straight sections of the APS storage ring that can accommodate insertion devices. It is proposed that the insertion devices in four of these straight sections will be similar to those built in the current phase. Such sectors are referred to as Type A Sectors. On the additional five straight sections of the storage ring, novel insertion devices capable of providing x-rays with unique characteristics will be built to perform special purpose scientific and technological research. These sectors are referred to as Type B Sectors in this document. 
Table 1.2.1

Status of Collaborative Access Teams (CATs) in the Current Phase (December 1992)

Letters of Intent Reviewed/Approved

$35 / 22$

CAT Proposals Reviewed by the PEB

CAT Proposals Approved

New Proposals under Preparation

APS Sectors Requested

Sectors Approved

Sectors Requested by the New Proposals

Sectors To Be Instrumented behind Shield

Wall in Current Construction

Total Principal Investigators

Number of Universities

Number of Industries

Number of Government Labs, etc.

Number of Medical Schools

Approximate Cost of Construction of Proposed Beamlines

Funds Committed by Industries

Funds Assured by DOE/Foundations/ States 
In this new initiative, the Type A Sectors will be completed first to meet the excess demand of the CATs approved during current phase. Once this demand is met, attention will be given to the special purpose beamlines that will occupy the five Type B Sectors. The technical subjects for these beamlines will not be decided at this time. However, this document discusses 12 possible subjects for such beamlines. As the new initiative matures, a decision will be made on the special purpose beamlines using the best expertise from the scientific and technological user comm nity of the APS.

\section{RE: REN CES}

1 G. K. Shenoy, P. J. Viccaro, and D. M. Mills, Characteristics of the $7 \mathrm{GeV}$ Advanced Photon Source: A Guide for Users, Argonne National Laboratory Report, ANL-88-9 (1.988).

2 Planning Study for Advanced National Synchrotron-Radiation Facilities, P. Eisenberger and M. L. Knotek, Chairmen, Sandia Laboratory Report (March 1984).

3 Major Facilities for Material Research and Related Disciplines, F. Seitz and D. Eastman, Chairmen, National Academy of Sciences, Washington, DC (1984).

4 Physics Throughout the 1990's: An Overview, W. Brinkman, Chairman, NRC Report, National Academy of Sciences, Washington, DC (1986).

5 Review of the National Research Council Report on Major Facilities for Materials Research, F. Stehli, Chairman, Energy Research Advisory Board Subcommittee, DOE (1987).

6 Review of the National Research Council Report on Physics Through the 1990 's, B. Ancker-Johnson, Chairperson, Energy Research Advisory Subcommittee, DOE (1987).

7 Report from the Secretary of Energy Advisory Board (SEAB) Task Force on ER Priorities, Charles Towns, Chairman, Washington, DC (1991).

8 Memorandum Dated November 27, 1991, from the Energy Secretary: FY 1994 Secretarial Planning Guidance,Washington, DC (1991), p.7-8. 


\section{CHAPTER II EXPERIMENTAL FACILITIES}

\section{INTRODUCTION}

In this section, a brief description of the experimental facilities is provided.

There are 40 straight sections on the Advanced Photon Source storage ring, of which 34 will be available for the use of insertion devices. The remaining six sections are reserved for the storage-ring hardware and diagnostics. The storage ring incorporates 80 bending magnets; however, only 35 will be available for extracíing radiation beams. One set of a bending magnet radiation port and an insertion device (ID) radiation port constitutes a sector. ${ }^{1}$

Generally, the ID photon beamline consists of four functional sections. The first section is the ID (or IDs) on a single straight section that provides the radiation source(s). In the case of a bending magnet beamline, the radiation is produced by the bending magnet.

The second section, immediately outside the storage ring but still inside the concrete shielding tunr.e!, is the front-end section of the beamline. This section contains the masks, apertures, safety shutters, photon beam position monitors, filters, window, etc., which confine, define, and control the photon beam.

The third and the fourth of the beamline are usually built by the user groups to their requirements but are suitably designed to meet all the APS safety and design requirements. In the third section, just outside the concrete shield wall and on the experiment floor, is the first optics enclosure (FOE). In a majority of experiments, the FOE contains optics for delivering filtered or monochromatic radiation. The crystal and/or mirror and/or multilayer optics contained in the FOE are designed to handle the expected radiation power loads from either the IDs or the bending magnet source. In a few cases, the FOE may be used to carry out white radiation experiments.

The fourth section of the beamline will vary widely depending on the nature of the investigations. Generally, it consists of beam transport, additional optics, apertures, filters, and, finally, the experimental station. This station contains the sample under investigation, radiation analyzer optics, detectors, etc., to characterize the scattering, imaging, or absorption processes. The experimental station will also contain various instruments to control the environment of the sample under study.

Typically, the third and the fourth sections of the beamline will occupy the experiment floor, which allows for a maximum of about a $70 \mathrm{~m}$ long beamline measured from the center of the ID straight section. Some applications will require much longer beamlines, extending out of the Experiment Hall. Such beamlines will require additional buildings located outside the Experiment Hall to support the instrumentation and data collection. There are at least two locations to place long 
beamlines ( $200 \mathrm{~m}$ to $1000 \mathrm{~m}$ ) that could extend beyond the Experiment Hall without any building obstructions.

In all, there can be at least $34 \mathrm{ID}$ beamlines and 35 bending magnet beamlines on this facility, assuming that only one section 3 and 4 is built on each of the radiation sources.

In the current construction phase of the APS project, funds are provided to develcp sections 1 (insertion device) and 2 (front end) of the beamlines in 16 sectors. These sectors, equipped with IDs and front ends, are referred to as Type A Sectors. At the present time, the number of user proposals for Type A Sectors exceeds 16 (16 ID and 16 bending magnet). Hence, additional Type A Sectors will be equipped behind the shield wall in the new initiative. The user groups are raising funds to build sections 3 and 4 for all the proposed sectors.

The intent of the APS Instrumentation Initiative is to enhance the experimental facility on the APS by installing an additional 9 ID beamlines and 9 bending magnet beamlines. In this chapter (Chapter II), the scope and conceptual design for the experimental facilities of the new initiative will be provided.

\section{GenERal Layout OF THE EXPERIMENTAL FACILITIES}

\subsection{Introduction}

The APS Instrumentation Initiative intends to extend the experimental facility on the APS by installing equipment behind the shield wall (IDs and front ends) in nine additional sectors. The new initiative will comprise four Type A Sectors and five Type B Sectors, the later containing more sophisticated insertion devices and front ends. A complement of 12 possible beamlines that may be built in Type B Sectors is described in this report. It should be understood that the exact nature of these beamlines to be placed on the experiment floor of the APS will be reevaluated at a later date to address the user community requirements prior to their construction.

\subsection{Disposition of Instrumentation Initiative Beamlines}

The beamlines in the current construction project occupy the following 16 sectors: 1 through 8 and 13 through 20 (see Fig. 2.2.1). The set of insertion devices that will support these beamlines will include planar undulator and wigglers. These devices are typically $2.5 \mathrm{~m}$ long, and the currently designed front ends are capable of handling the power and power density from such devices.

In the new initiative, there are many users who will build their beamlines based on the above set of insertion devices and supporting front ends. Thus, such sectors, referred to as Type A Sectors, will also be built in the APS Instrumentation Initiative. The number of Type A Sectors in the new initiative will be limited to four, bringing the total number of Type A Sectors to 20. The placement of the new 


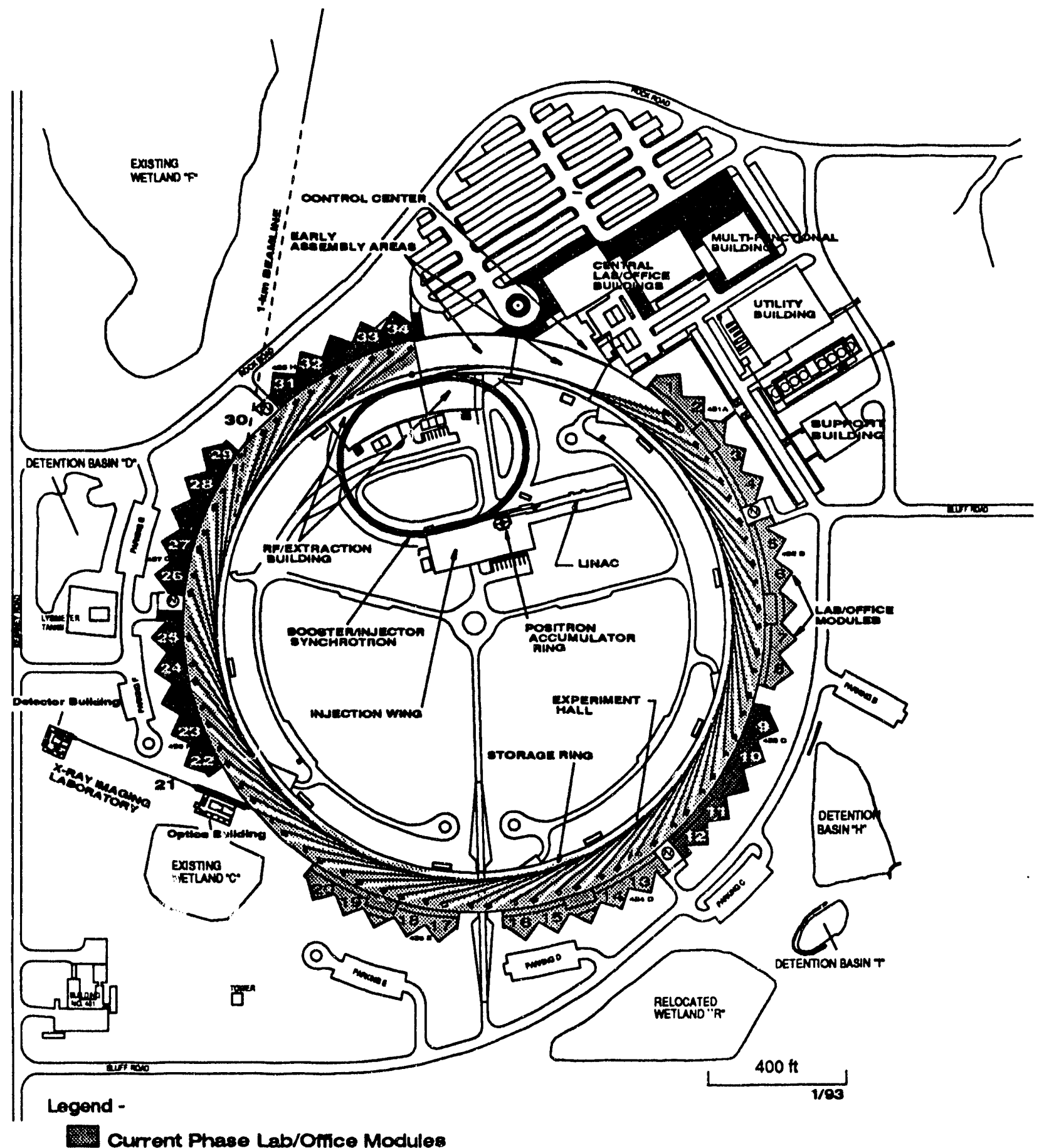

Instrumentation Initiative Lab/Ofice Modules

Fig. 2.2.1 Disposition of sectors at the APS. In the new initiative, Sectors 9 through 12 will be Type A Sectors. The Type B Sectors will be Sectors 21 through 26. 
Type A Sectors on the experiment floor is likely to occupy Sectors 9 through 12 (see Fig. 2.2.1).

The nature of the remaining beamlines that will be built as part of the Instrumentation Initiative will be distinct from those occupying Type A Sectors. The sectors that are occupied by these beamlines will be referred to as Type B Sectors and will contain a different class of insertion devices. Some of these could be 5-m-long undulators or super-conducting wigglers; others could be IDs producing $\mathrm{x}$-rays with distinct types of polarization or multiple IDs on a single straight section to deliver two $x$-ray beams with differing characteristics, etc. Corresponding beamline front ends will be capable of handling the power from these unique devices and will satisfy new functions required to perform specific research programs on these beamlines. Tentatively, it is assumed that these five Type B Sectors will start at Sector number 21 and extend through 25 to complete the facility.

To support the users operating their beamlines on a total of 9 (4 Type $A$ and 5 Type B) sectors to be built in the new initiative, it is necessary to provide Laboratory/Office Modules (LOMs). In the APS Instrumentation Initiative, provision has been made to build four LOMs to support the users from 16 sectors. These are similar to those provided in the on-going construction phase. One additional $\mathrm{x}$-ray imaging laboratory is based on a Long Beamline extending outside the Experiment Hall. This is discussed in Chapter III.

\section{INSERTION DEVICES}

\subsection{Introduction}

The Advanced Photon Source will be a powerful source of high-brilliance hard $\mathrm{x}$ rays with energies above $1.0 \mathrm{keV}$. In addition to the availability of bending magnet (BM) radiation, which has a critical energy of $19.5 \mathrm{keV}$, undulator and wiggler insertion device (ID) sources can be introduced on 34 straight sections on the storage ring. The unique spectral properties and expected flexibility in the ID operation will support the APS users in the current construction phase. Proposed new techniques utilizing the full potential of ID sources will be the focal point of research for many users of the Instrumentation Initiative beamlines. These will include operation of IDs in the storage-ring vacuum, production of radiation with enhanced coherence and unique polarization properties, IDs designed to generate very high energy $\mathrm{x}$-rays $(>50 \mathrm{keV}$ ), and storage-ring timing synchronization to perform temporal studies.

In Type A Sectors, the IDs are composed of two sets of magnet arrays in a planar geometry that produce a spatially oscillating magnetic field along the length of the device. In Type B Sectors, more complex IDs are planned as a part of the complement of IDs to be built in the APS Instrumentation Initiative. In these Type $\mathrm{B}$ IDs, there will be unique placements of magnet arrays to produce elliptical or helical magnetic fields. The arrays are typically made of permanent magnets with high permeability magnetic poles. In special situations, either electromagnets or superconducting magnets will be used. 
The spectral properties of the devices are related to the peak magnetic field, $B_{0}$, in the mid-plane of the two arrays in a planar ID (to be provided in a Type A Sector). The resulting amplitude of oscillating motion of the particle beam and the maximum slope angle of the trajectory depend linearly on both $B_{0}$ and the period of the device, $\lambda_{0}$, through the deflection parameter, $K$, defined by

$$
\mathrm{K}=0.933 \lambda_{0}(\mathrm{~cm}) \mathrm{B}_{0}(\text { Tesla)} \text {. }
$$

For $\mathrm{K}$ less than approximately ten, the maximum slope angle is $\theta=\mathrm{K} / \gamma$, where $\gamma$ $=13699$, the relativistic mass enharcement for the $7-\mathrm{GeV}$ positron. This is to be compared with the natural opening angle of synchrotron radiation, $\psi \approx 1 / \gamma$, which is approximately $73 \mu \mathrm{rad}$ for the $7-\mathrm{GeV}$ APS storage ring.

The spectral properties of a planar device will depend on the relative values $\theta$ and $\psi$. In the undulator regime, where $K \approx 1$, the radiation from each part of the trajectory is within the radiation opening angle $\psi$. This results in spatial and frequency bunching that gives rise to a typical undulator spectrum consisting of narrow energy bands of radiation called harmonics. The energy of the harmonics is dependent on the energy of the positron and the magnetic field experienced by the positron. Typically, the radiative divergence at the harmonic energy is a fraction of the natural opening angle, $\psi$, and the photon density at this energy is enhanced.

In a planar wiggler, where $K \geq 5$, the output from the device is the sum of intensities from each magnetic pole, and the spectral output is similar to that from an equivalent $B M$ but is contained within a horizontal angular range of $\pm K / \gamma$. The spectral output on axis is approximately $\mathrm{N}$ times the output from an equivalent $\mathrm{BM}$ source, where $\mathrm{N}$ is number of magnetic poles in the wiggler.

The spatial and angular distribution of the $7-\mathrm{GeV}$ positron beam will affect the undulator spectrum most severely. Because the positrons in the beam are independent, the effective source size and angular distribution are a convolution of the radiative and particle beam distribution parameters.

The capability to design IDs that can provide x-rays with different polarization properties is as yet a new area, which will be exploited in the APS Instrumentation Initiative. Typically, the polarization properties are derived and varied by appropriate summation of phases of two linearly polarized $\mathrm{x}$-ray beams derived from two planar undulators placed orthogonally relative to each other (referred to as crossed undulators). The polarization properties are also obtained by controlling the trajectory of the positron through the complex IDs using different magnet configurations.

In Type B Sectors, complex insertion devices involving four magnet arrays providing the oscillatory field in the $\mathrm{x}$ and $\mathrm{y}$ directions will be built. In these IDs, 
the magnetic field vector experienced by the positron moving in the $\mathrm{z}$ direction is given by

$$
B=e_{x} B_{x_{0}} \cos \left(2 \pi z / \lambda_{0}\right) \pm e_{y} B_{y_{0}} \sin \left(2 \pi z / \lambda_{0}\right),
$$

and the deflection parameter is given by

$$
\mathrm{K}_{\mathrm{X}, \mathrm{y}}=0.933 \mathrm{~B}_{\mathrm{Xo}, \mathrm{yo}} \lambda_{\mathrm{O}} \text {. }
$$

Based on the values of $K_{x}$ and $K_{y}$, the positron will move with relativistic velocities either in a helical or an elliptical trajectory. Hence, depending on the relative values of $\mathrm{K}_{\mathbf{x}}$ and $\mathrm{K}_{\mathrm{y}}$, one can generate undulator or wiggler radiation with different polarization contents.

Additional IDs with different capabilities have also been planned for Type B Sectors and will be presented below.

\subsection{Insertion Devices in Type A Sectors}

Several IDs have been identified as standard x-ray sources for Type A Sectors. These include two planar undulators and two planar wigglers. 2 Undulator A, which has Nd-Fe-B permanent magnets and vanadium permendur poles to form a hybrid geometry, is capable of spanning the photon energy interval from 4.7 to about 40 $\mathrm{keV}$ using the first- and third-harmonic radiation. Undulator $\mathrm{B}$, which also has the hybrid structure, is tunable from approximately 13 to $20 \mathrm{keV}$ using its firstharmonic radiation. Wiggler $\mathrm{A}$, with the hybrid geometry, and Wiggler $\mathrm{B}$, with a magnetic structure based on electromagnets, have critical energies $\left(\mathrm{E}_{\mathrm{c}}\right)$ of 32.6 and $9.8 \mathrm{keV}$, respectively. These critical energies are above and below that for the bending magnet radiation of $19.5 \mathrm{keV}$. Figure 2.3.1 shows a comparison of the onaxis brilliance of the BM source to that of Wiggler A and Undulator A sources.

\subsubsection{Undulators}

The design parameters for Undulator A have been well developed. As a part of the study, Monte-Carlo simulations of the radiation properties of these IDs have

been performed. ${ }^{3}$ A summary of the properties of these IDs is given in Table 2.3.1. Figures 2.3.2-4 show the energy spectra of radiation from Undulator A for three values of $K$.

The planar geometry of this undulator has a hybrid configuration in which the magnetic field strength and distribution depend on the geometry of the pole tips. The field quality is also determined by the magnetic and geometric quality of the magnetic material. In arriving at the magnetic structure of this device, various geometric parameters of the structure are iteratively optimized through a twodimensional field computation (yz plane). The resulting parameters are presented in Table 2.3.2. Three-dimensional (3D) effects are estimated in order to obtain a pole width and a magnet width that provide the required field homogeneity. 
Figure 2.3.5 shows the optimized two-dimensional magnetic flux lines of a quarter period of the 3.3-cm-period undulator at gaps of 1.15 and $3.00 \mathrm{~cm}$. The pole piece has been chamfered at $45^{\circ}$ to avoid both flux saturation at the corners of the pole tip and the demagnetization of the permanent magnet material.

Both Undulators A and B will support the special needs of the user community, namely, the capability to generate "broad-band" undulator radiation. This is accomplished by tapering the two magnetic arrays of a planar undulator along the $z$ axis. This concept has already been tested by the APS Experimental Facilities staff and will be incorporated in all the devices constructed for Type A Sectors.

The undulator assemblies have magnetic field end-correctors. A detailed analysis of the geometry of the end-correctors and the 3D effects that determine the pole widths is being experimentally evaluated as a part of the R\&D program. Also, the actual measurements on a 5-cm-period undulator built for the Advanced Light Source (ALS) will provide a solid basis for improving the end-corrector design and the pole width specification.

In Table 2.3.3, partial specifications for the periodic magnetic structure of Undulator $A$ and $B$ are presented. These specifications assure sufficient dynamic aperture for the storage-ring lattice to provide its optimal performance.

It should be pointed out that the APS Experimental Facilities staff has collaborated with a Cornell group to design, specify, commission, and test a 3.3-cmperiod undulator prototype. Also, a second prototype with a 7.5-cm period has been designed, specified, and tested by the APS Experimental Facilities staff at the National Synchrotron Light Source. Through these successful activities on prototype undulators, it has been assured that there are no technical difficulties in constructing such devices to required specifications.

As a part of the design activity in the current phase, Undulators A and B are now being fully specified with regard to their design tolerance requirements. A number of Undulators A and B will be procured during FY 1993-94 to complete the current phase construction and will be commissioned and operated during FY 199596. This experience should greatly enhance our capability to build the undulators in the APS Instrumentation Initiative. 


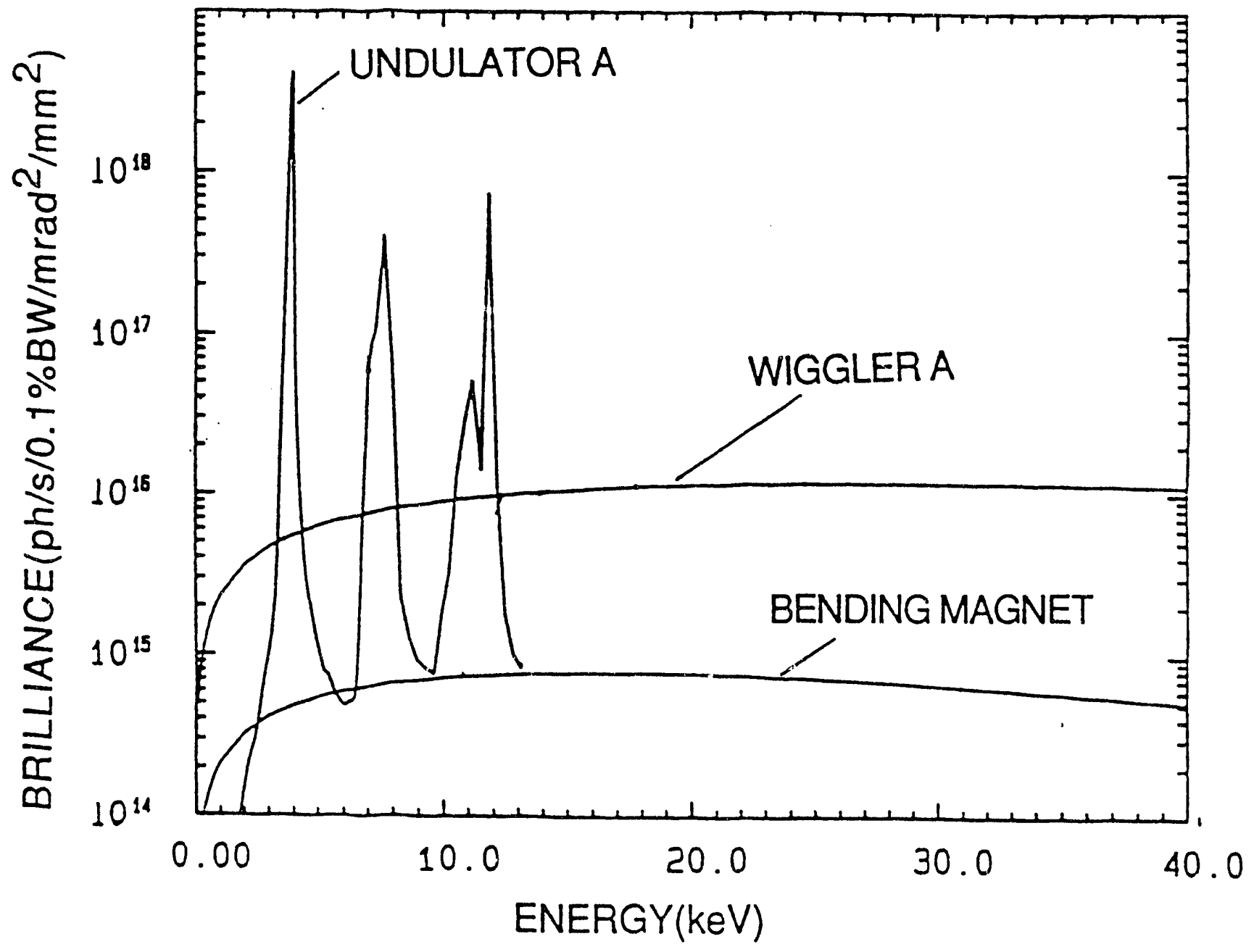

Figure 2.3.1 A comparison of the on-axis brilliance for the APS bending magnet source, Wiggler A, and Undulator A 
Table 2.3.1

Summary of Insertion Devices for Type A Sectors

\begin{tabular}{|c|c|c|c|c|}
\hline & \multicolumn{2}{|c|}{ Undulators } & \multicolumn{2}{|c|}{ Wigglers } \\
\hline & A & B & A & B \\
\hline Period (cm) & 3.3 & 2.1 & 15 & 25 \\
\hline Number of Periods & 72 & 114 & 10 & 10 \\
\hline Length (m) & 2.5 & 2.5 & 1.5 & 2.5 \\
\hline $\begin{array}{c}\text { Undulator 1st-Harmonic } \\
\text { Minimum (keV) } \\
\text { Maximum (keV) }\end{array}$ & $\begin{array}{l}4.7 \\
14\end{array}$ & $\begin{array}{l}13 \\
20\end{array}$ & & \\
\hline $\begin{array}{l}\text { Wiggler Critical Energy } \\
\mathrm{E}_{\mathbf{c}}(\mathrm{keV})\end{array}$ & & & 32.5 & 9.8 \\
\hline $\mathrm{K}_{\max }$ & 2.5 & 1.1 & 14 & 7 \\
\hline
\end{tabular}



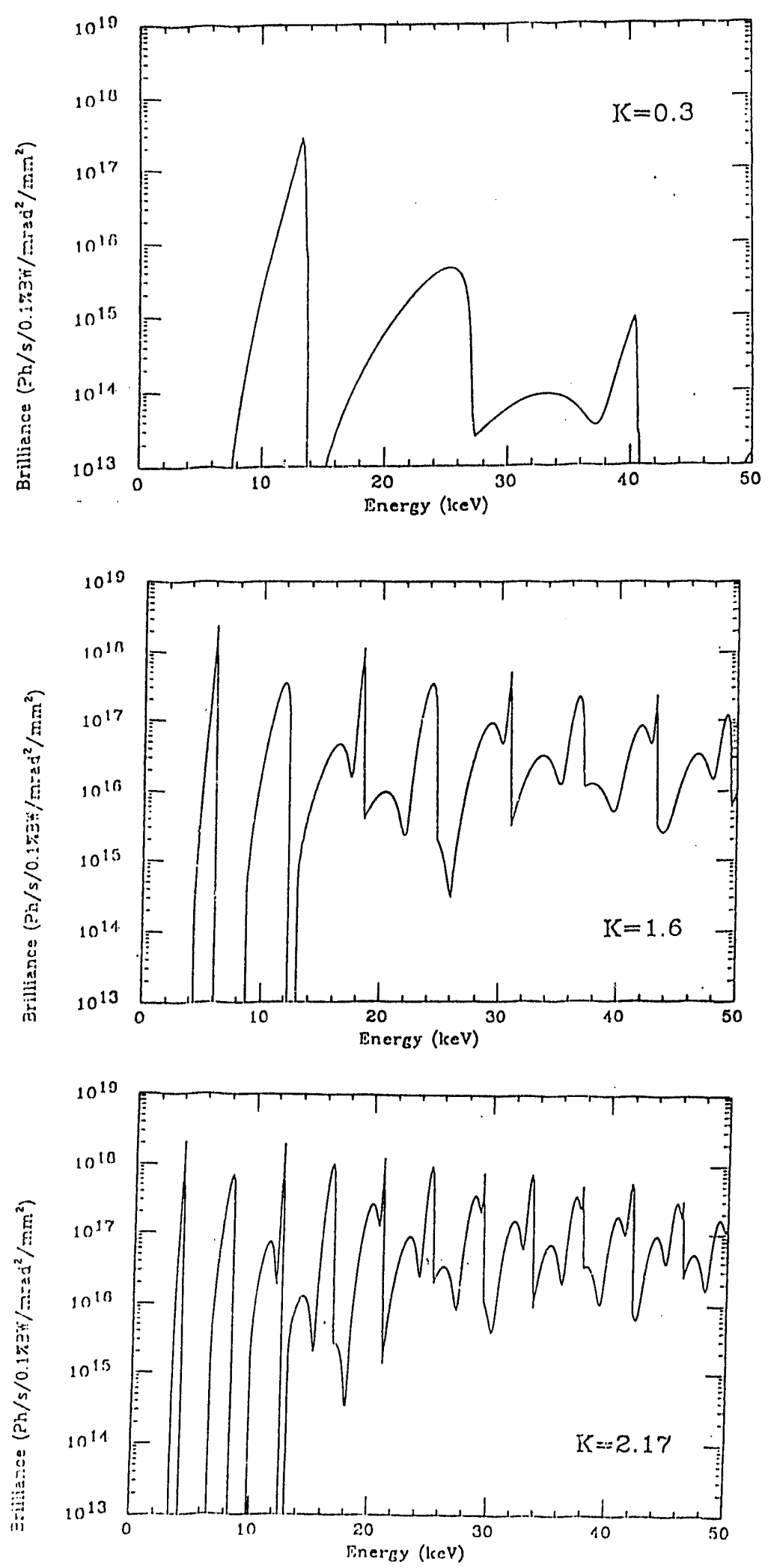

Figures 2.3.2 - 2.3.4 Energy spectra of radiation from Undulator A for three values of $K$. The positron beam parameters in these calculations are: horizontal beam width $\sigma_{x}^{\prime}: 308 \mu \mathrm{m}$, vertical beam height $\sigma_{y}^{\prime}: 85 \mu \mathrm{m}$, horizontal beam divergence $\sigma_{x}^{\prime}: 24 \mu \mathrm{rad}$, vertical beam divergence $\sigma_{\mathrm{y}}: 9 \mu \mathrm{rad}$ 


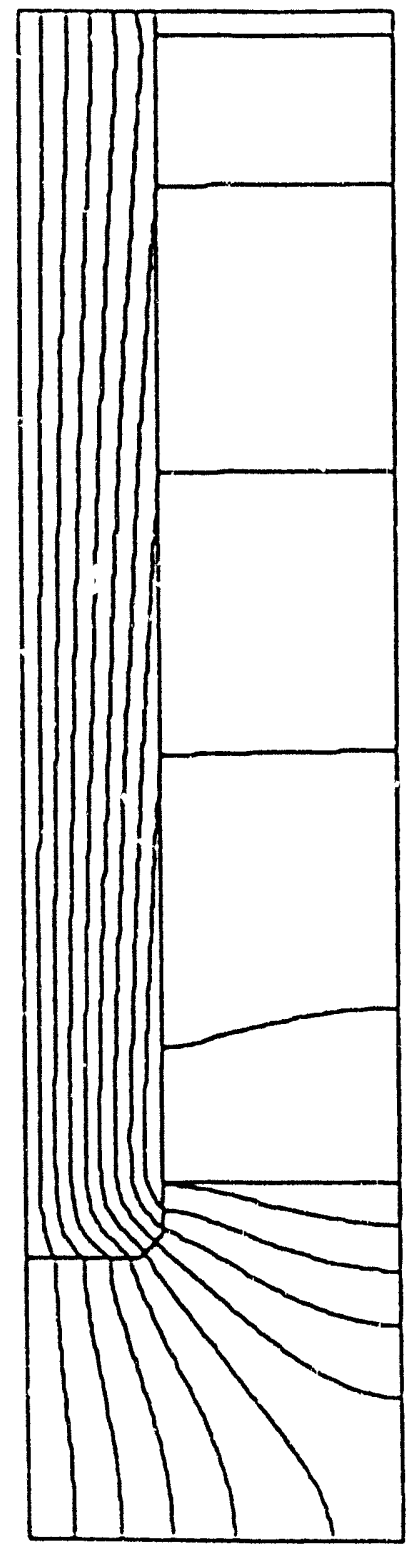

cap: $11.50 \mathrm{~mm}$

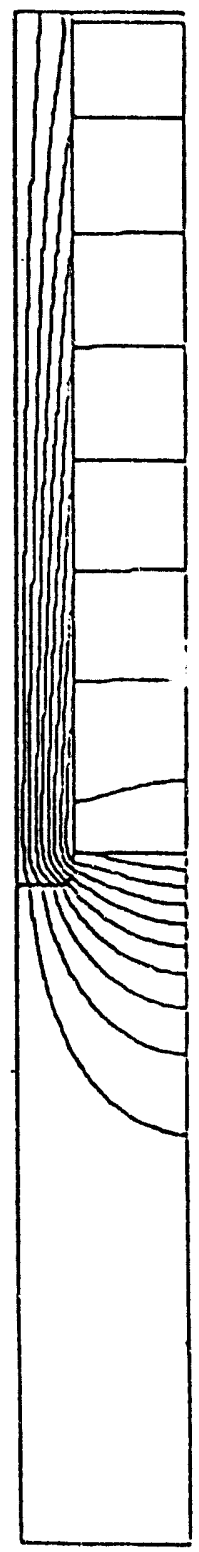

Gap: $30.00=$

Figure 2.3.5 Optimized 2D magnetic flux profile of a quarter period of Undulator A 
Table 2.3.2

Optimized Design Parameters of Nd-Fe-B Hybrid Undulators A and B

\begin{tabular}{|c|c|c|}
\hline & Undulator $\mathrm{A}$ & Undulator B \\
\hline Undulator Period (cm) & 3.3 & 2.1 \\
\hline $\begin{array}{l}\text { Minimum }(\mathrm{cm}) \\
\text { Maximum }(\mathrm{cm})\end{array}$ & $\begin{array}{l}1.15 \\
3.0\end{array}$ & $\begin{array}{l}1.15 \\
3.0\end{array}$ \\
\hline Pole Width- x Direction (cm) & 6.00 & 4.0 \\
\hline Pole Height - y Direction (cm) & 6.00 & 2.4 \\
\hline Pole Thickness - z Direction (cm) & 0.56 & 0.42 \\
\hline Magnet Width - x Direction (cm) & 7.80 & 5.2 \\
\hline Magnet Height - y Direction (cm) & 6.85 & 3.0 \\
\hline Magnet Thickness - $\mathrm{z}$ Direction (cm) & 1.10 & 0.73 \\
\hline $\begin{array}{r}\text { Pole Tip Overhang - y Direction (cm) } \\
\text { - x Direction (cm) }\end{array}$ & $\begin{array}{l}1.00 \\
0.90\end{array}$ & $\begin{array}{l}0.7 \\
0.7\end{array}$ \\
\hline Peak Field on Axis - Maximum (G) & 6917 & 3700 \\
\hline
\end{tabular}

Table 2.3.3

Partial List of Specifications for Undulators A and B

\begin{tabular}{lr}
\hline Parameter & Specification \\
\hline Maximum Random Field Error (\%) & 0.48 \\
Gap Resolution $(\mu \mathrm{m})$ & 2.5 \\
Transverse Roll-Off at $\pm 1 \mathrm{~cm}(\%)$ & 0.5 \\
Total Steering Errors $(\mathrm{G}-\mathrm{cm})$ & $<100$ \\
Integrated Quadrupole $(\mathrm{G})$ & $<10$ \\
Integrated Sextupole $(\mathrm{G} / \mathrm{cm})$ & $<100$ \\
\hline
\end{tabular}




\subsubsection{Wigglers}

The prerequisite for wigglers is a large $\mathrm{K}$-value, which will result in a continuous and wide energy spectrum. This is achieved by having either large $B_{0}$ and/or large $\lambda_{0}$. The critical energy of the spectrum can be made either large or small by proper choice of peak magnetic field. In Table 2.3.4, design parameters for two APS wigglers planned for Type A Sectors are given. Wiggler A has a high critical energy, while Wiggler $B$ is typical of low-critical-energy devices. In various scientific proposals from approved user groups, both these wigglers have been requested. Wiggler A can be constructed with the use of permanent magnets in a hybrid configuration, while the construction costs for Wiggler $B$ would be lower if electromagnetic technology is employed.

\subsubsection{Insertion Device Vacuum Chambers for Type A Sectors}

The ID vacuum chamber provides a straight section continuum of the storage ring enabling the positron beam to pass through the mid plane of the ID. The trajectory of the beam through the ID results in an intense $x$-ray beam. The straight section ID vacuum chamber requires a vacuum level of 10-10 Torr, similar to that of the storage ring. Poorer vacuum can result in unacceptably high production of bremsstrahlung radiation from the scattering of the positron beam from gas molecules.

The design of the vacuum chambers for the Type A Sectors has received considerable attention during the current phase design studies. The tolerance specifications are a very critical element of this design study. Prototype chambers have been built and tested as part of the current phase R\&D studies, and the information obtained will provide the necessary input for the Instrumentation Initiative construction activities.

The overall length available in each sector of the storage ring for the ID vacuum chamber is $5.2 \mathrm{~m}$. This length excludes the transition section between the storage ring vacuum chamber and the ID chamber. Because the devices on Type A Sectors will generally be $2.5 \mathrm{~m}$ long, in the final design the length of ID vacuum chamber may be limited to this length with the remaining part of the straight section being occupied by the standard storage ring vacuum chamber.

The ID vacuum chamber has a cross section similar to that of the main storage ring chamber but with smaller overall dimensions. A cross-sectional view of such a chamber is shown in Fig. 2.3.6. The ID chamber consists of an elliptical positron beam chamber connected to a pumping antechamber (containing NEG strips) through a narrow channel. The material of choice for the chamber is a 6063-T5 aluminum alloy, and the geometry is achieved through an extrusion process.

The positron beam chamber is situated in the mid-plane region of the upper and lower magnet arrays of the ID. The maximum magnetic field developed by the ID magnets depends on the minimum gap. This gap over the length of the ID and its dimensional tolerance determine the maximum magnetic field achievable for an ID. For reasons of energy tunability, it is desirable to reach the largest field 


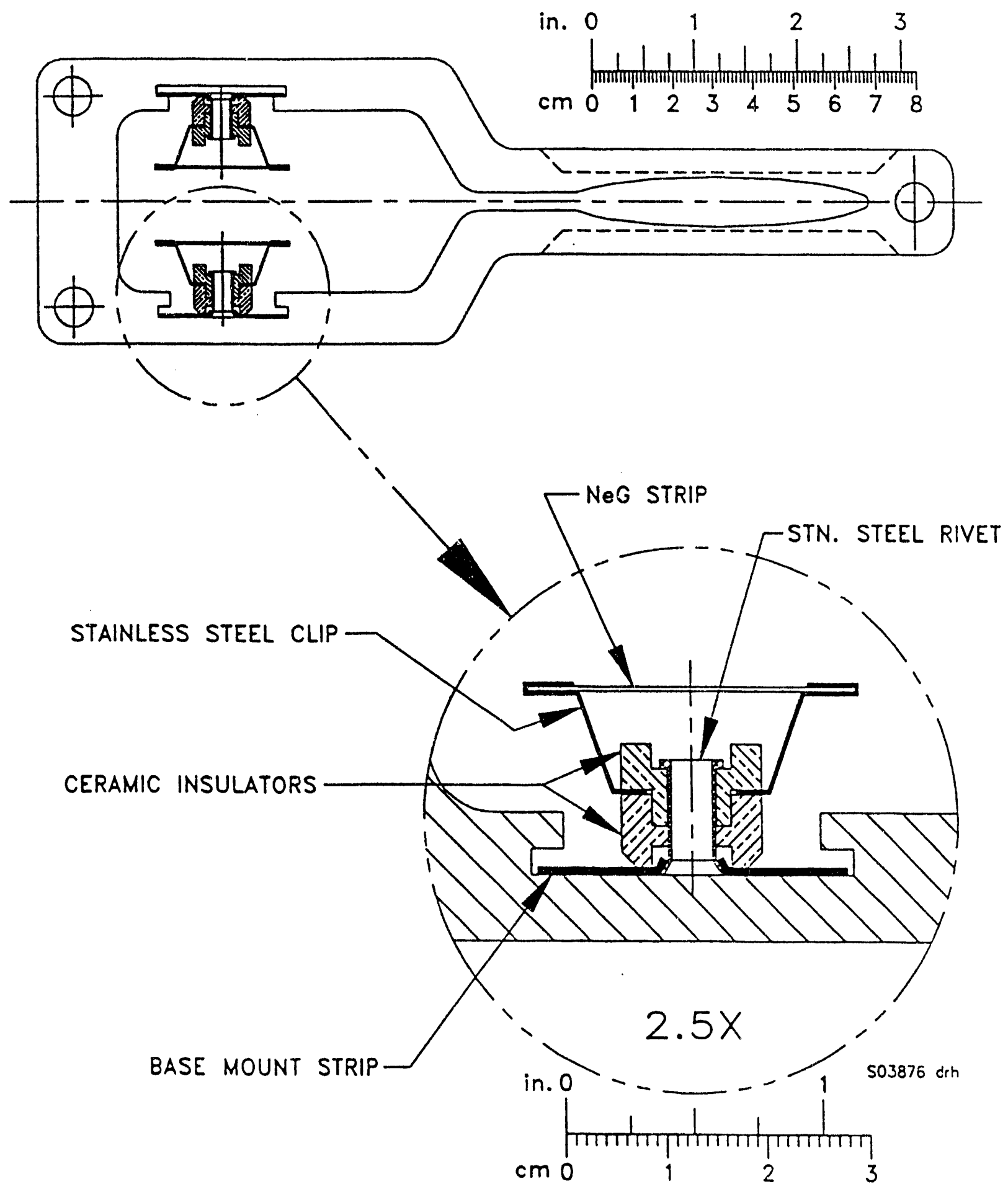

Figure 2.3.6 Cross section of the ID vacuum chamber 
possible at the minimum gap. For the ID chambers to be installed in the APS Instrumentation Initiative, the nominal minimum dimension of the positron beam chamber is $10 \mathrm{~mm}$. A detailed analysis of various mechanical tolerances leads to a total tolerance budget of $1.5 \mathrm{~mm}$ for the vacuum chamber and its supporting structure. With the manufacturing technologies available, this tolerance should be easily achievable. This ID vacuum chamber will lead to a minimum magnetic gap of $1.15 \mathrm{~cm}$ for the ID, which fully supports the spectral requirements from all the insertion devices.

Table 2.3.4

Design Parameters for Wigglers A and B

\begin{tabular}{lll}
\hline Parameters & Wiggler A & Wiggler B
\end{tabular}

Peak Field on Axis (G) 1000

Gap (cm)

1.55

1.55

Period (cm)

15.0

25.0

Bending Radius (m)

23.3

78.0

K

Number of Periods

10

10

Length (m)

1.5

2.5

Critical Energy (keV)

32.6

9.8

Structure

Nd-Fe-B

Electromagnet

Hybrid

Flux at Critical Energy

$10^{14}(\mathrm{ph} / \mathrm{s} / 0.1 \% \mathrm{BW} / \mathrm{mrad})$

2.2

2.3 


\subsection{Insertion Devices in Type B Sectors}

\subsubsection{Introduction}

The trend in the development of insertion devices is to produce radiation with characteristics suitable for the next generation of scientific research programs. The very nature of the APS with the capability to introduce newer types of devices on the straight sections permits the flexibility to grow with newer ideas and requirements of the user community. Some of the ideas for the APS Instrumentation Initiative are now being discussed in the community. A short list of radiation properties that may be included in the Instrumentation Initiative beamlines focuses on

- the capability to realize $20-50 \mathrm{keV}$ first-harmonic radiation from undulators,

- the need to obtain polarized radiation from $0.5 \mathrm{keV}$ to $100 \mathrm{keV}$ with variable ellipticity and helicity,

- very high photon energy $(\approx 50-200 \mathrm{kE}$ ) raciiaiiùin,

- two undulator sources for a single investigation,

- longer undulators $(5 \mathrm{~m})$ to produce higher brightness sources.

Clearly, this list will grow and change with increasing user focus on the APS Instrumentation Initiative in the coming years. In the following sections, the current thinking in the development of insertion devices for the Type B Sectors to be instrumented as a part of the Instrumentation Initiative construction is described.

\subsubsection{In-Vacuum Undulators}

The desire for in-vacuum undulators is dictated by a variety of needs. A primary one is to realize high energy first-harmonic radiation. For a $7-\mathrm{GeV}$ particle, the first-harmonic radiation is given by

$$
\mathrm{E}(\mathrm{keV})=46.55 /\left\{\lambda_{0}(\mathrm{~cm}) \times\left[1+\mathrm{K}^{2} / 2\right]\right\} .
$$

To produce first harmonic energy of $50 \mathrm{keV}$, the undulator period should be smaller than $1 \mathrm{~cm}$. However, such short undulator periods require a narrow gap between the magnet arrays of the undulator, typically $0.5 \mathrm{~cm}$, in order to generate an adequate magnetic field.

In the undulators planned for Type A Sectors, the minimum gap is fixed at 1.15 $\mathrm{cm}$ by the vacuum chamber in the straight section, and the magnet arrays of the undulator are outside the vacuum chamber. The stored positron beam experiences the undulator magnetic field through the wall of the vacuum chamber. Thus, a limit is set on the minimum gap by the geometry of the vacuum chamber. 
Consequently, the minimum and the maximum first-harmonic energies are limited to about 15 and $22 \mathrm{keV}$, respectively.

If the magnet arrays of the undulator can be brought into the storage-ring vacuum, the capability of operating small gap undulators is enhanced. An additional bonus would be that the minimum operational aperture for the storage ring can be defined by the type of operation (e.g., commissioning, injection, top-off mode, etc.). Undulators that will operate in the storage-ring vacuum will be referred to as in-vacuum undulators.

In-vacuum devices need special consideration with respect to material selection and device construction. The proposed design will consist of a vacuum chamber roughly $50-80 \mathrm{~cm}$ in diameter in which the mechanical assembly with the magnet arrays is arranged so that the gap can be varied precisely. The motion to the magnet arrays is provided from the outside of the vacuum chamber using linear motion feed-throughs and bellows. The undulator will be supported by a support stand located outside the vacuum chamber. The vacuum inside this large chamber, typically $3 \mathrm{~m}$ long for a 2.5 -m-long undulator, is achieved using a conventional pumping system made of NEG strips and ion pumps.

The following special considerations will be given to the design and development of such devices.

- The magnet blocks will contain no epoxy in their forming.

- The magnet blocks will be plated with $\mathrm{Ni}$ to avoid degassing.

- Magnet arrays will be mounted on $\mathrm{Al}$ strong-backs using the clamping techniques developed for the APS prototypes.

- UHV requirements inside the vacuum chamber must be evaluated along with the production of bremsstrahlung radiation from the positron beam interaction with the residual molecules.

- For UHV operation of the device, the baking procedure should be carefully developed with the capability to magnetize the magnet blocks in vacuum after the baking process.

- Special attention will be paid to avoid discontinuities in the rf coupling between the stored beam and the wall current paths. Such discontinuities can lead to heating of the magnets and the vacuum chamber, resulting in the disruption of operation. The design will minimize parasitic mode losses in the vacuum chamber containing the insertion device.

- Techniques of performing the magnetic measurements on a device in vacuum need to be developed.

The first 4-cm-period in-vacuum undulator has recently been built and operated in the TRISTAN Accumulator Ring at KEK at $6.5 \mathrm{GeV} .4$ 


\subsubsection{Circular Polarization Sources}

\subsubsection{Helical Undulator}

Many insertion device sources have been proposed in the literature for the production of circularly polarized radiation. A helical undulator is perhaps a classic example in which there are two sets of magnet arrays producing orthogonal fields, $B_{x}$ and $B_{y}$, such that they are $\pi / 2$ out of phase. When $K_{x}=K_{y}$, one has perfect helical motion of the positron, which results in the circularly polarized radiation on axis.

The difficulty with such devices is with regard to the minimum gap achievable both in the vertical and the horizontal directions. The APS storage-ring apertures have been designed to permit a narrow vertical gap allowing one to use short period IDs. On the other hand, the horizontal aperture for the APS storage ring is large, which makes it difficult to achieve small ID gap in the horizontal direction and, hence, small ID periods. As a result, the only possibility of using helical undulators is with long-period configurations that can only produce low energy radiation. It should be noted that such helical undulators with two sets of magnet arrays in the $x$ and y direction can be used to produce circularly polarized hard x-rays if the entire device is built in vacuum as discussed for a planar device in Section 3.1.

A helical undulator can, however, be built with a planar geometry as shown in Fig. 2.3.7.5 In this geometry, periods as small as a few centimeters can be achieved, which will produce circularly polarized radiation in the $5-15 \mathrm{keV}$ range at the APS. An undulator with this design can be easily built and will be included in the Instrumentation Initiative plans.

\subsubsection{Elliptical Multipole Wiggler}

Another device of considerable interest is the elliptical multipole wiggler. The principle of such a device is illustrated in Fig. 2.3.8, and a conceptual configuration is shown in Fig. 2.3.9. This wiggler consists of one set of magnet arrays that produces a large vertical magnetic field $\left(B_{y}\right)$ and a second set of magnet arrays that produces a small horizontal field $\left(B_{X}\right)$. The two fields $B_{X}$ and $B_{y}$ are $\pi / 2$ out of phase. The resulting field is helical with different radii in the horizontal and vertical planes. When $\mathrm{K}_{\mathrm{y}}>>1$ and $\mathrm{K}_{\mathrm{x}} \approx 1$, the on-axis radiation is circularly polarized. The radiation is effectively a superposition of the off-plane radiation from a bending magnet source, because half the period of the positron orbit can be regarded as the orbit through the bending magnet. The intensity of the on-axis radiation from such a wiggler is $2 \mathrm{~N}$ times higher than that from a bending magnet, $\mathrm{N}$ being the periods in the wiggler.

The choice of $\mathrm{K}_{\mathrm{X}}$ is crucial for generating both a high degree of polarization $\mathrm{P}_{c}$ and high brightness. Large values of $\mathrm{K}_{\mathrm{x}}$ will lead to a nearly unit value for $\mathrm{P}_{\mathrm{c}}$; however, in that case, the on-axis brightness will be small. The elliptical multipole wiggler to be built in the APS Instrumentation Initiative will have $\mathrm{K}_{\mathrm{y}} \approx 15$ and $\mathrm{K}_{\mathrm{X}} \approx 1$. Such a device with $\lambda=15 \mathrm{~cm}$ will have a critical energy of about $32 \mathrm{keV}$ 


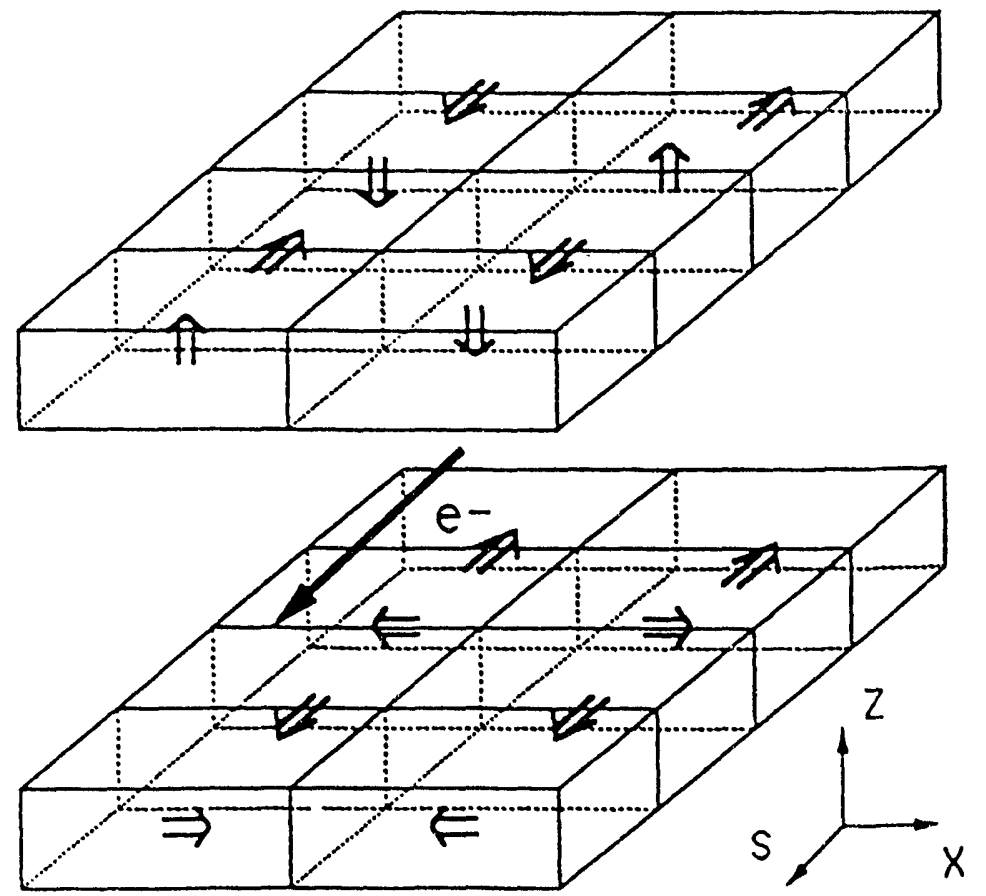

Figure 2.3.7 A possible magnet design for a helical undulator with a planar geometry and made of rectangular pieces of permanent magnet. The arrow shown corresponds to the orientation of the magnetization (Ref. 5) 


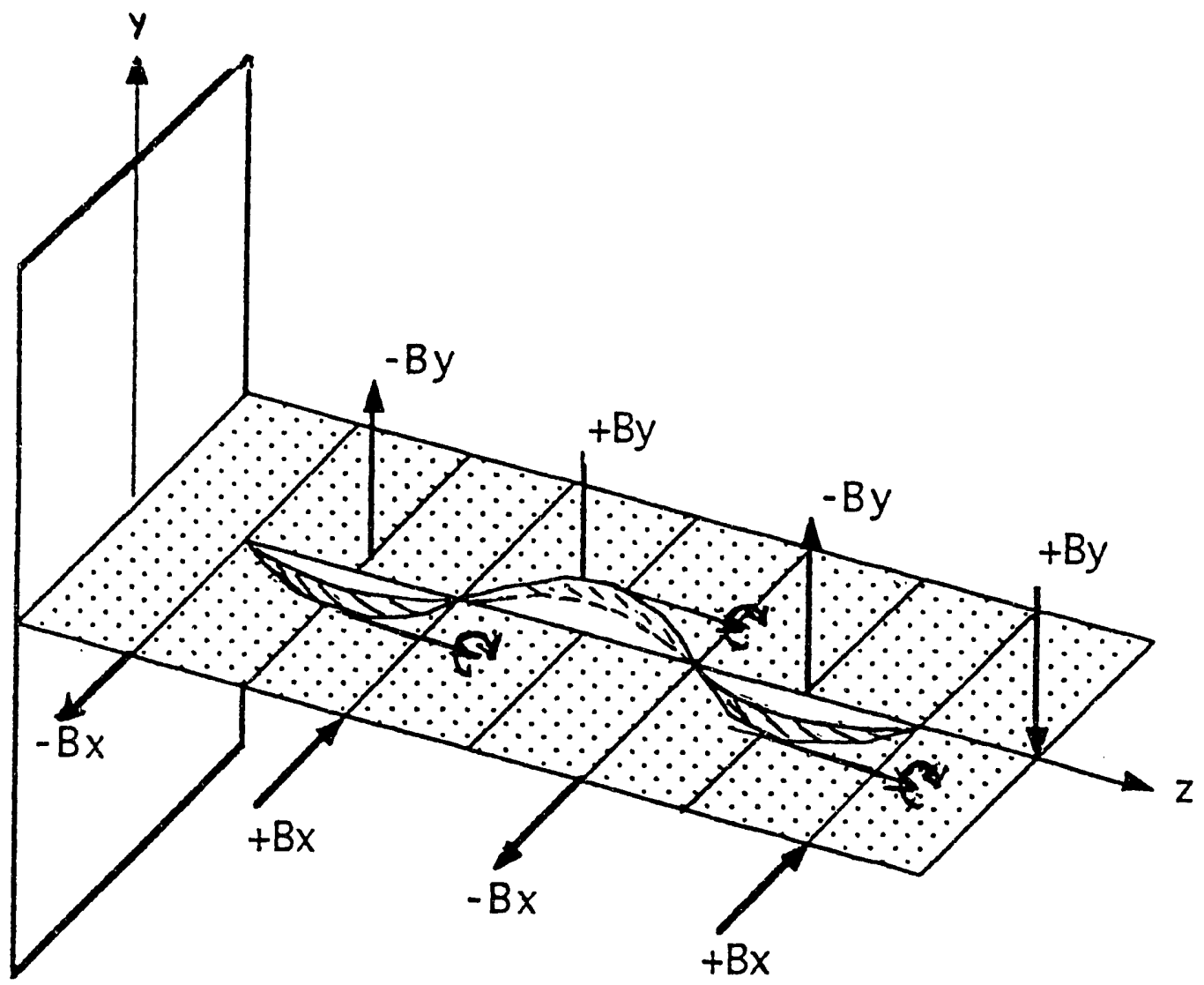

Figure 2.3.8 Placement of magnetic field components to generate circularly polarized radiation from the elliptical motion of positrons in a wiggler. The $B_{X}$ and $B_{y}$ components have a special phase difference of $\pi / 2$ along the $z$ axis. In this case, the on-axis radiation is right circularly polarized (Ref. 7) 


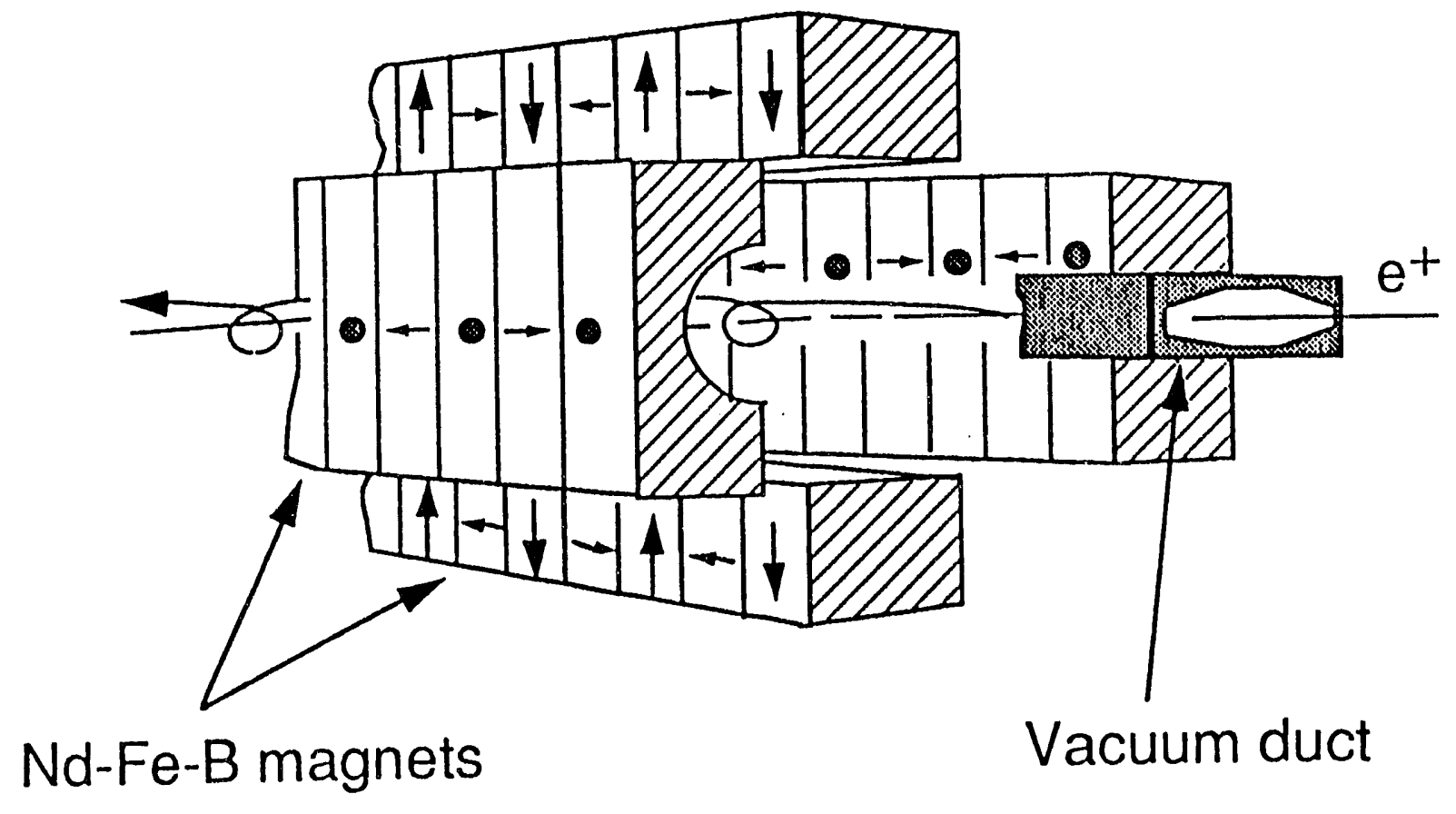

Figure 2.3.9 Conceptual design for the elliptical multipole wiggler 
and a $P_{c}$ value of about 0.9 over a wide energy range. In Fig. 2.3.10, the variation of $P_{c}$ as a function of photon energy for this device is shown for various values of $K_{\mathbf{x}}$. The on-axis relative intensity of the circularly polarized beam as a function of photon energy for various values of $\mathrm{K}_{\mathrm{X}}$ is shown in Fig. 2.3.11.6 $\mathrm{K}_{\mathrm{X}}$ will be chosen depending on the nature of the experiment. A device of this type has been tested on the Accumulator Ring at TRISTAN.7

It is equally important to note that the value of $P_{c}$ can be degraded by the vertical beam divergence $\sigma_{y}{ }^{\prime}$, or by large experimental angular acceptance, $\theta$. To minimize such degradations, the following equation should be satisfied:

$$
\mathrm{K}_{\mathrm{x}}>\gamma\left[\sigma_{\mathrm{y}}{ }^{2}+(\theta / 2)^{2}\right]^{1 / 2} .
$$

Use of pinholes placed along the beamline can achieve $\theta \ll \sigma_{y}{ }^{\prime}$. For the APS, $\gamma \sigma_{y}{ }^{\prime}$ is 0.12 , and, hence, the above condition is easily satisfied for all values of $K_{X}=0.2$.

The translation of the set of $x$-magnet arrays relative to $y$-magnet arrays in this device will produce different states of polarization. For example, if the two sets of magnet arrays are in phase, linearly polarized radiation will be produced. On the other hand, if the phase angle between the two sets of magnet arrays is either $+\pi / 2$ or $-\pi / 2$, the on-axis radiation will be either right or left circularly polarized. The phase change can be accomplished by translating the horizontal magnet array from $-\lambda / 4$ to $+\lambda / 4$.

The ability to switch between various polarization states in a single investigation will be of considerable importance. This switching would demand the translation of one set of magnet arrays relative to the other set along the $z$ direction. However, it is difficult to accomplish such mechanical movements on a short time scale because of strong magnetic interactions between the two sets of magnet arrays. One way of switching the state of polarization is by employing electromagnets in the horizontal magnet arrays. Such a device will be capable of switching the circular polarization state between right- and left-handedness within milliseconds. The design of the horizontal electromagnetic arrays will need detailed considerations on reducing eddy currents.

\subsubsection{Wigglers with Large Critical Energies}

The need for very high energy photons $(50-200 \mathrm{keV})$ is anticipated in many new scientific programs involving both elastic and inelastic $\mathrm{x}$-ray scattering. The $7-\mathrm{GeV}$ positrons provide a unique capability to meet this demand by choosing either a permanent magnet wiggler or a superconducting magnet wiggler. The parameters for such devices are discussed below. 


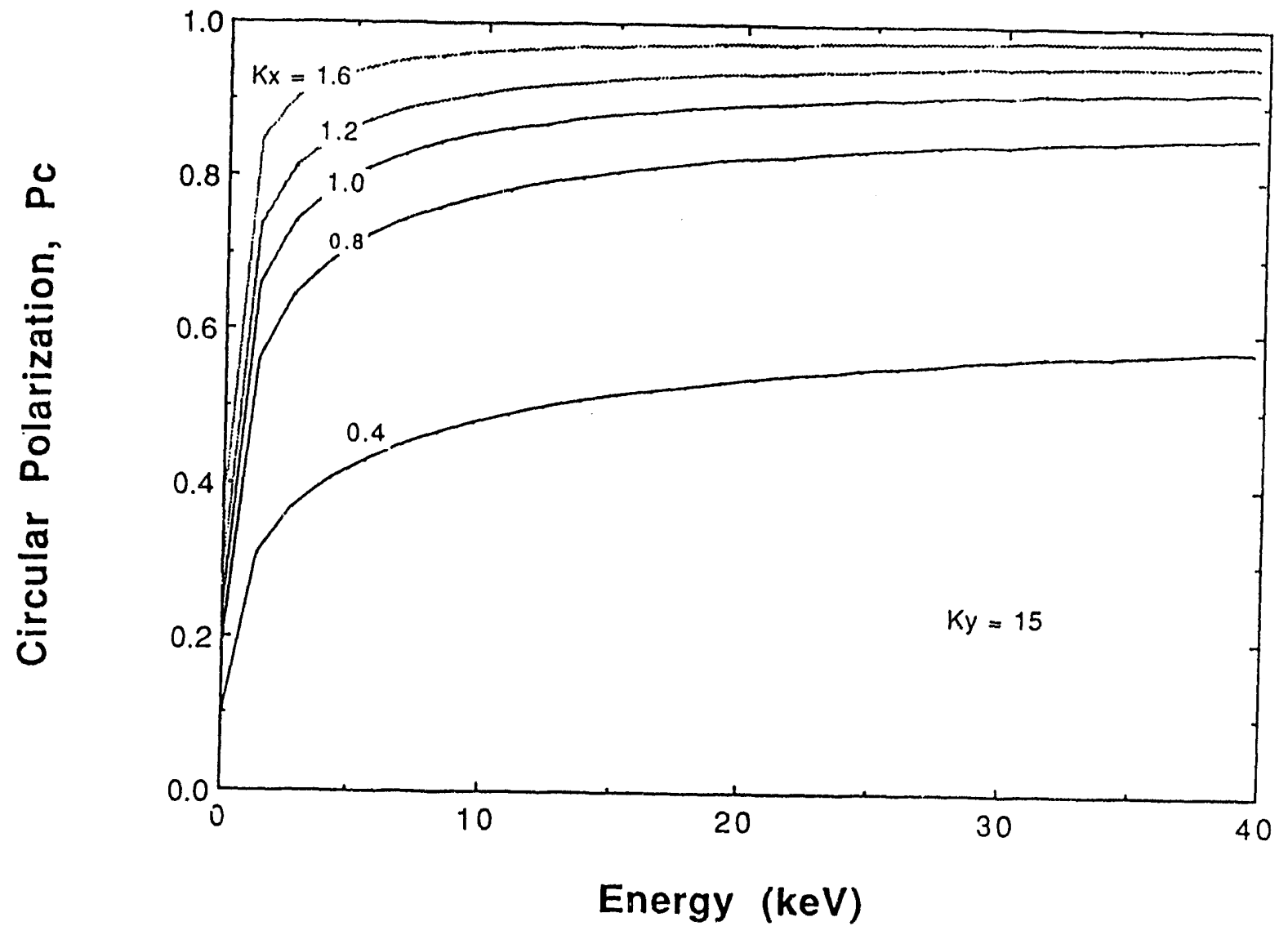

Figure 2.3.10 Variation of $P_{c}$ as a function of photon energy for the elliptical multipole wiggler with a period of $15 \mathrm{~cm}$ operated at $7 \mathrm{GeV}$ for $\mathrm{K}_{\mathrm{y}}=15$ and various
values of $\mathrm{K}_{\mathrm{X}}$ 


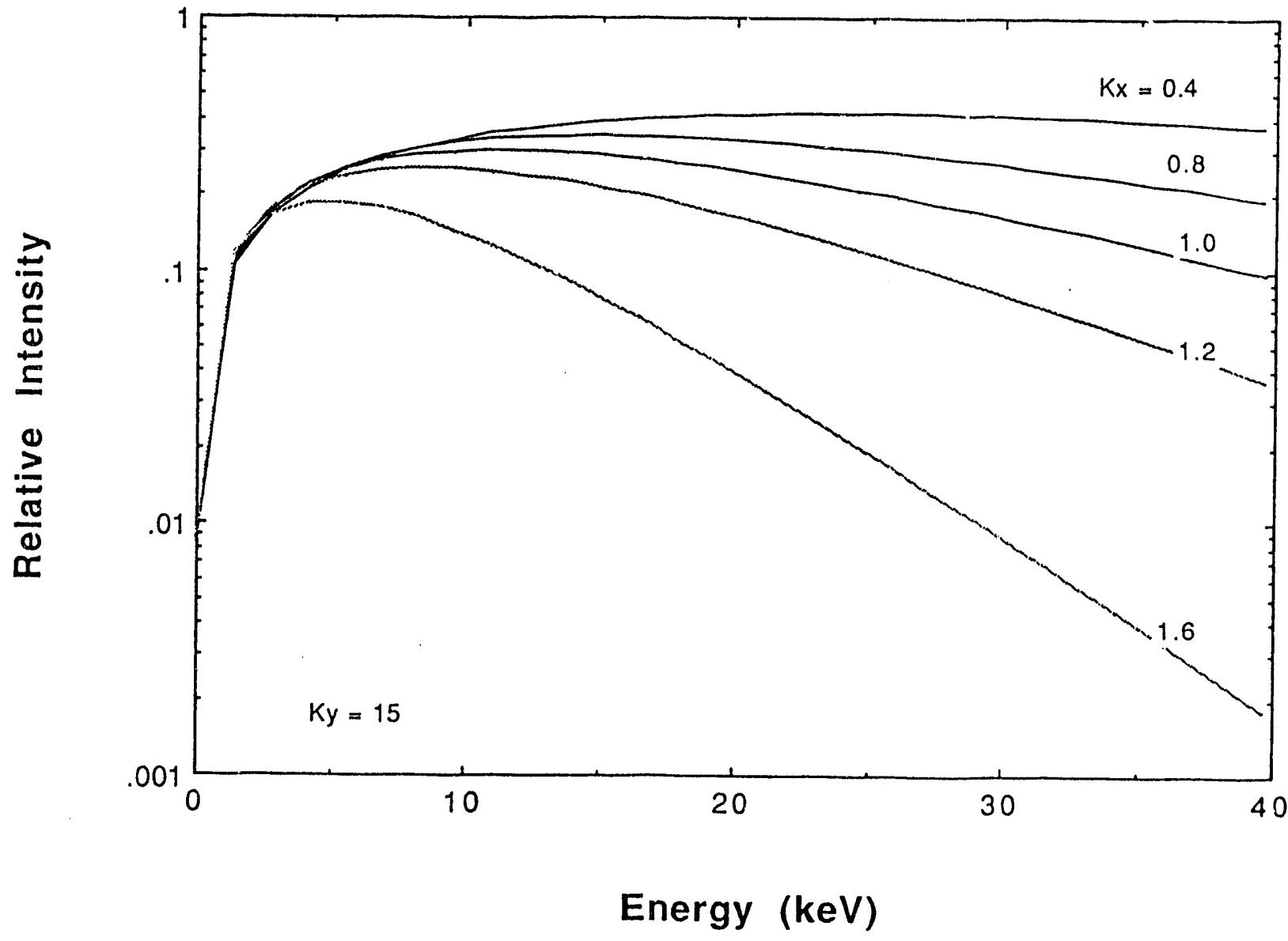

Figure 2.3.11 On-axis relative intensity of the circularly polarized beam as a function of photon energy for various values of $K_{X}$ 
The permanent magnet device will be based on a Nd-Fe-B hybrid configuration. A device with a magnetic period of $12 \mathrm{~cm}$ and a gap of $1.2 \mathrm{~cm}$ produces the required field to generate phritons with a critical energy of $58 \mathrm{keV}$.

With the clirent experience of building superconducting magnets with conventional magnet technology, a "single wiggle" device is chosen. This is depicted in Fig. 2.3.12. The device will be capable of providing a $3 T$ field and photons with a critical energy of about $98 \mathrm{keV}$. The device consists of three poles, the two end poles being twice the length of the central pole with one quarter of the field. The field from the central pole will be uniform over approximately $7.0 \mathrm{~cm}$, and the gap will be about $5.0 \mathrm{~cm}$. The total length of the device will be about $1 \mathrm{~m}$.

In Table 2.3.5, a summary of the parameters for both these wigglers is provided, and, in Fig. 2.3.13, the brilliance is plotted as a function of the photon energy for both these wiggler sources.

Table 2.3.5

Parameters for Wigglers Designed for the APS Instrumentition Initiative

Parameter

\section{Permanent Magnet Wiggler}

Supercinducting Magnet

Wiggler

Peak Field (T)

1.78

Berding Radius (m)

12.98

7.70

Gap (cm)

1.2

5.0

Period (cm)

12.0

Number of Poles

40

1 (+ 2 half-poles)

K

20

20

Device Length (m)

2.4

1.0

Critical Energy (keV)

58

98

Flux at

Critical Energy ${ }^{a}$

44.7

1.1

a In units of $10^{13} \mathrm{ph} / \mathrm{s} / 0.1 \% \mathrm{BW} / \mathrm{mrad} / 100 \mathrm{~mA}$ 


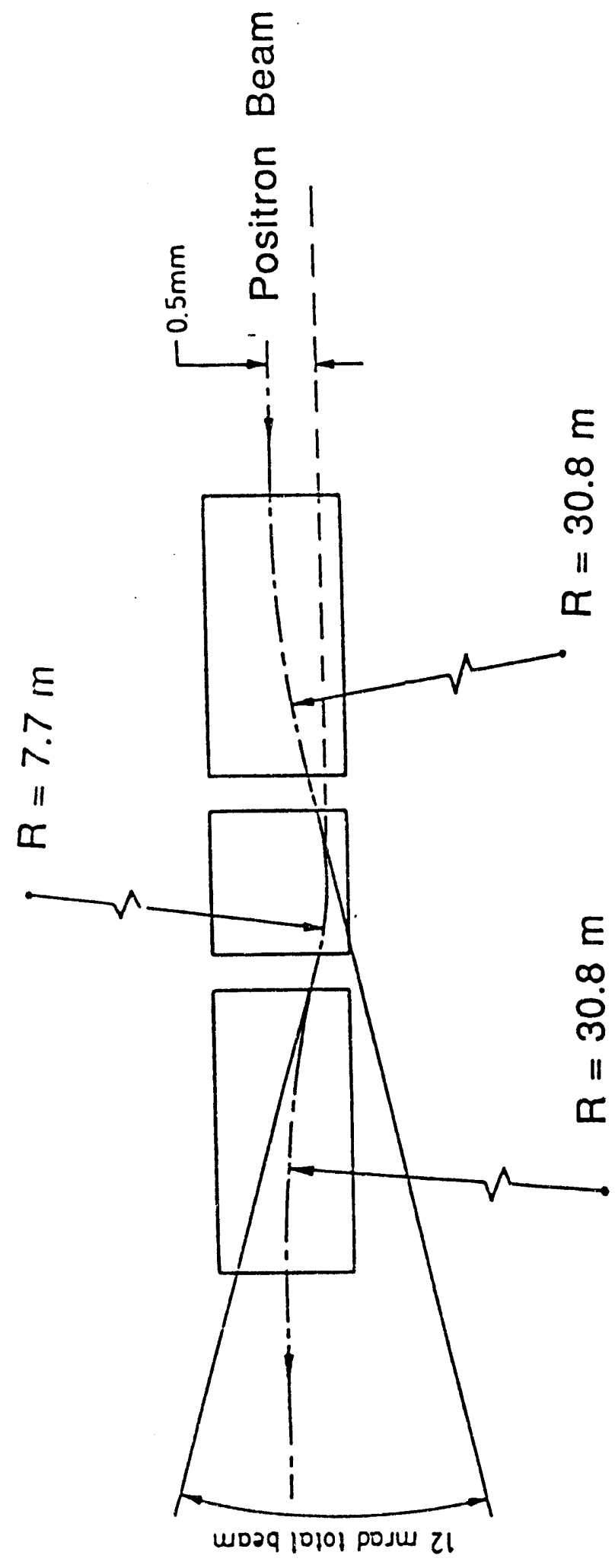

疍要

"ळ

웅

ㅁํㄹ

需表

$\infty+$

옹

造.

000

㐘舟

을

무

웡

串

要

죽귱 옹

웡

ฮㄴㅇㅇ

웡

\%

그응

\%

옹

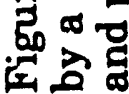




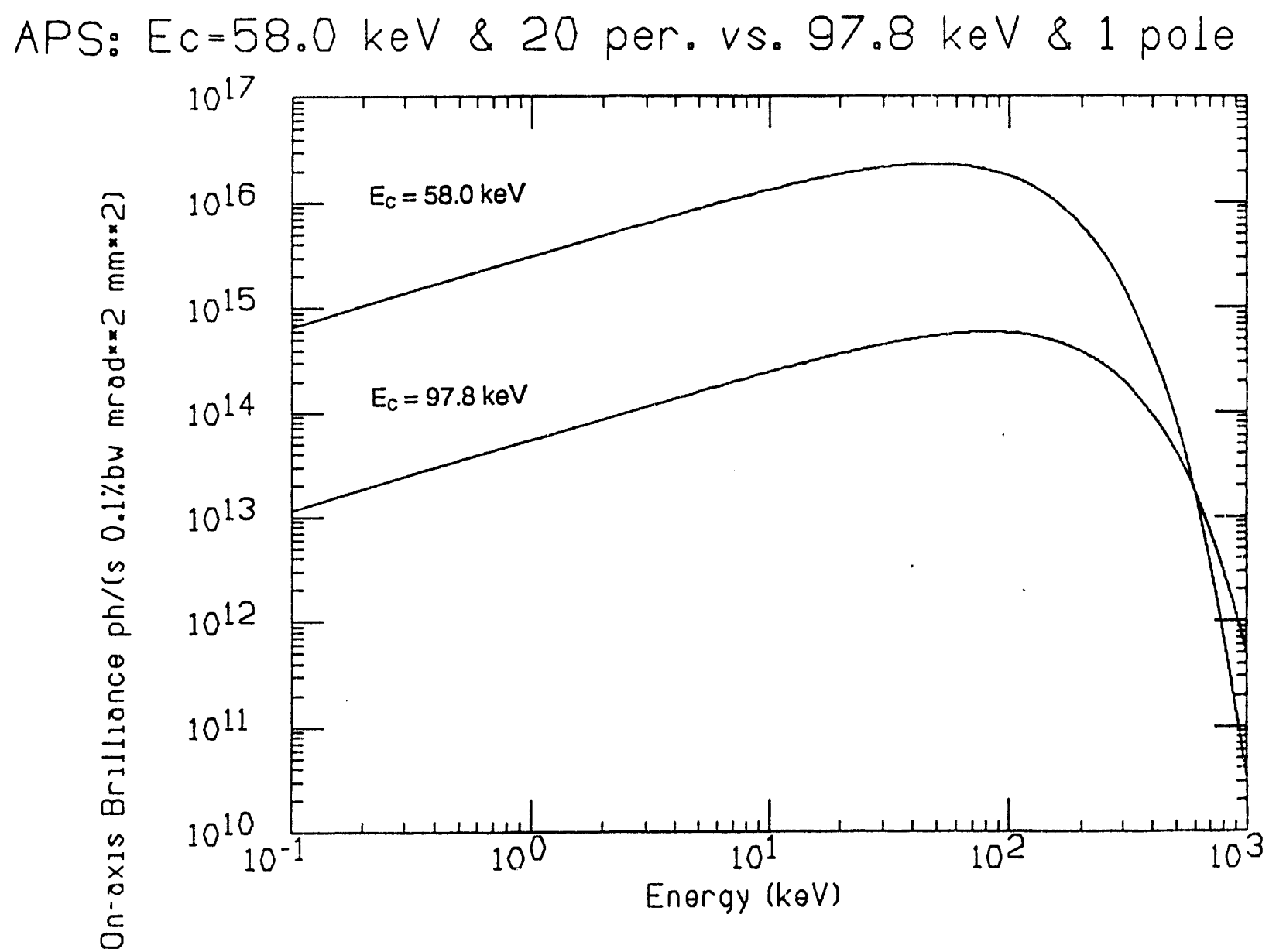

Figure 2.3.13 Brilliance as a function of photon energy for the permanent magnet wiggler $\left(\mathrm{E}_{\mathrm{c}}=58\right)$ and the superconducting wiggler $\left(\mathrm{E}_{\mathrm{c}}=98\right)$ 


\subsubsection{Double Undulator Sources on a Single Straight Section}

It is now recognized that there is a need to have two radiation sources providing two $x$-ray beams to a single experiment at a single beamline. Typical scientific programs with such a need are those based on three-dimensional holography and pump-probe temporal studies.

The basic intent is met by having two 2.5-m undulator sources located on a single 5.4-m-long straight section. The axes of the two undulators will make a small angle with respect to each other, so that the two radiation beams can be independently treated by required optics on the same beamline. In order that the positron beam travels along the true axes of otherwise canted undulators, the trajectory will have to be corrected at various positions in this straight section. But because the angle between the two undulator axes is typically on the order of a hundred microradians, there will be insignificant perturbation on storage ring operation.

The undulators needed for the 3D holographic imaging work will deliver 1-8 keV radiation. In this, one source will act as the reference beam while the other will illuminate the sample being investigated. The two devices on the straight section will be identical with a magnetic period of $20 \mathrm{~cm}$. Each of these devices will be use the Nd-Fe-B hybrid configuration. The spectral properties of such a device are shown in Fig. 2.3.14 for two typical $\mathrm{K}$ values of 2.7 and 1.0. The success of 3D holographic imaging will result from the capability of these undulator sources for delivering a very large coherent flux. In Fig. 2.3.15, the coherent flux from each of the devices is plotted as a function photon energy.

The second application of double radiation sources delivering two beams to one beamline will be for a pump-probe experiment. In this, one beam will illuminate (pump) the sample under study, and the second beam will analyze (probe) the outcome of the illumination from the first source. Most of these studies are carried out in time domain, and, hence, the two beams will be synchronized with respect to each other in a specific fashion to meet the experimental requirements using the timing sequence of the positron bunches in the storage ring. It is possible that the two sources will be required to deliver radiation beams of different characteristics depending on the nature of investigation. It is important to realize that the two devices will be so configured in the straight section that the radiation beams from them, after necessary manipulations by the x-ray optics, will converge at the same spot on the sample under study. It is anticipated that the devices to be used for these studies will be Type A Undulators, the same as those designed and operated during the current construction phase.

For some Instrumentation Initiative beamlines, the brilliance of a $5-\mathrm{m}$ undulator would be essential. This can be accomplished by aligning two $2.5-\mathrm{m}$ undulators along the z-axis to operate on a single straight section. The total brilliance of radiation from two such segmented undulators will be less than that from a single 5-m undulator. Because the brilliance of APS undulator radiation is dominated by the phase space dimensions of the positron beam in the hard $\mathrm{x}$-ray 


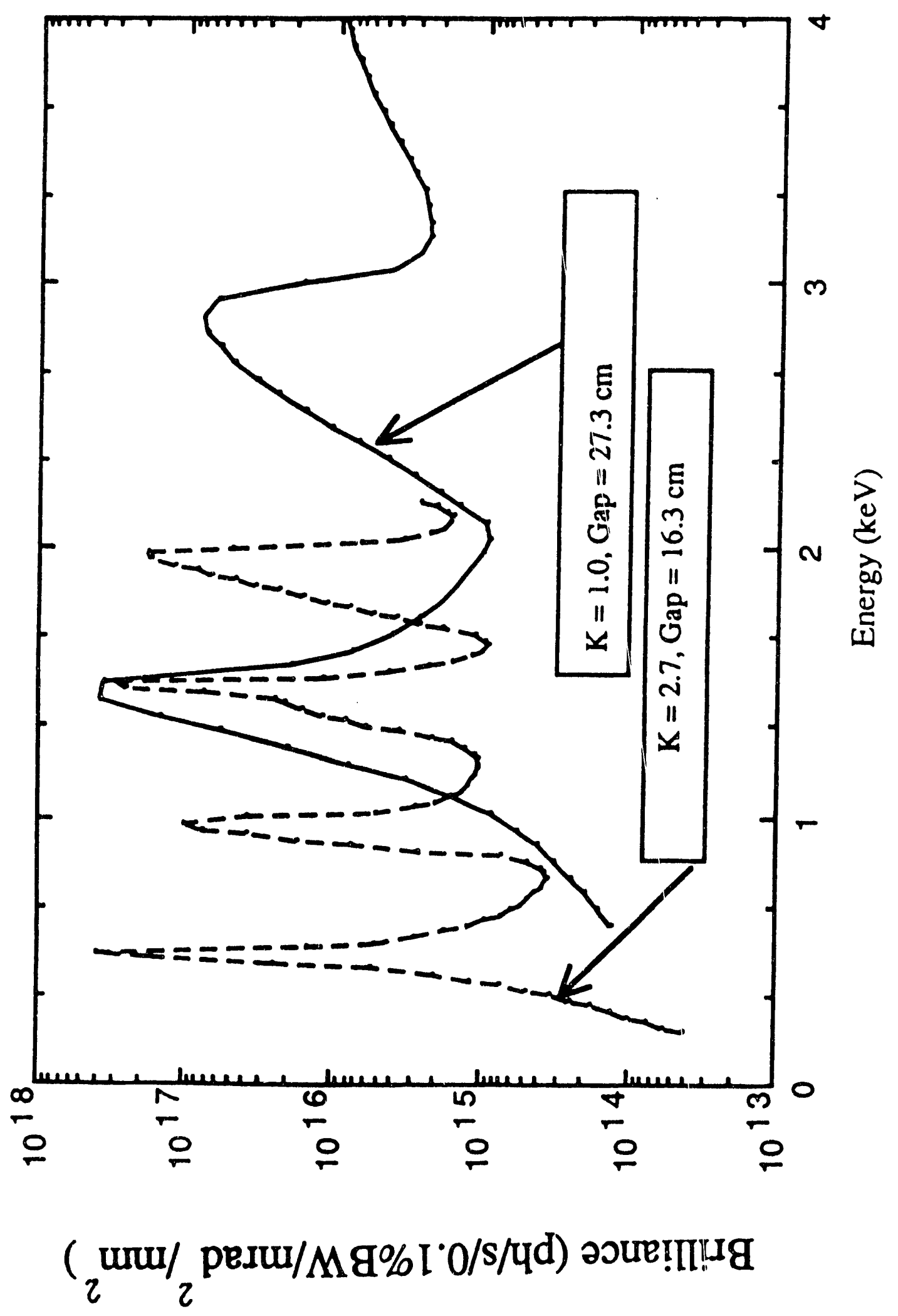

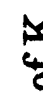

3

른

$+$

.

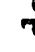

롱

P

क्ष

选 


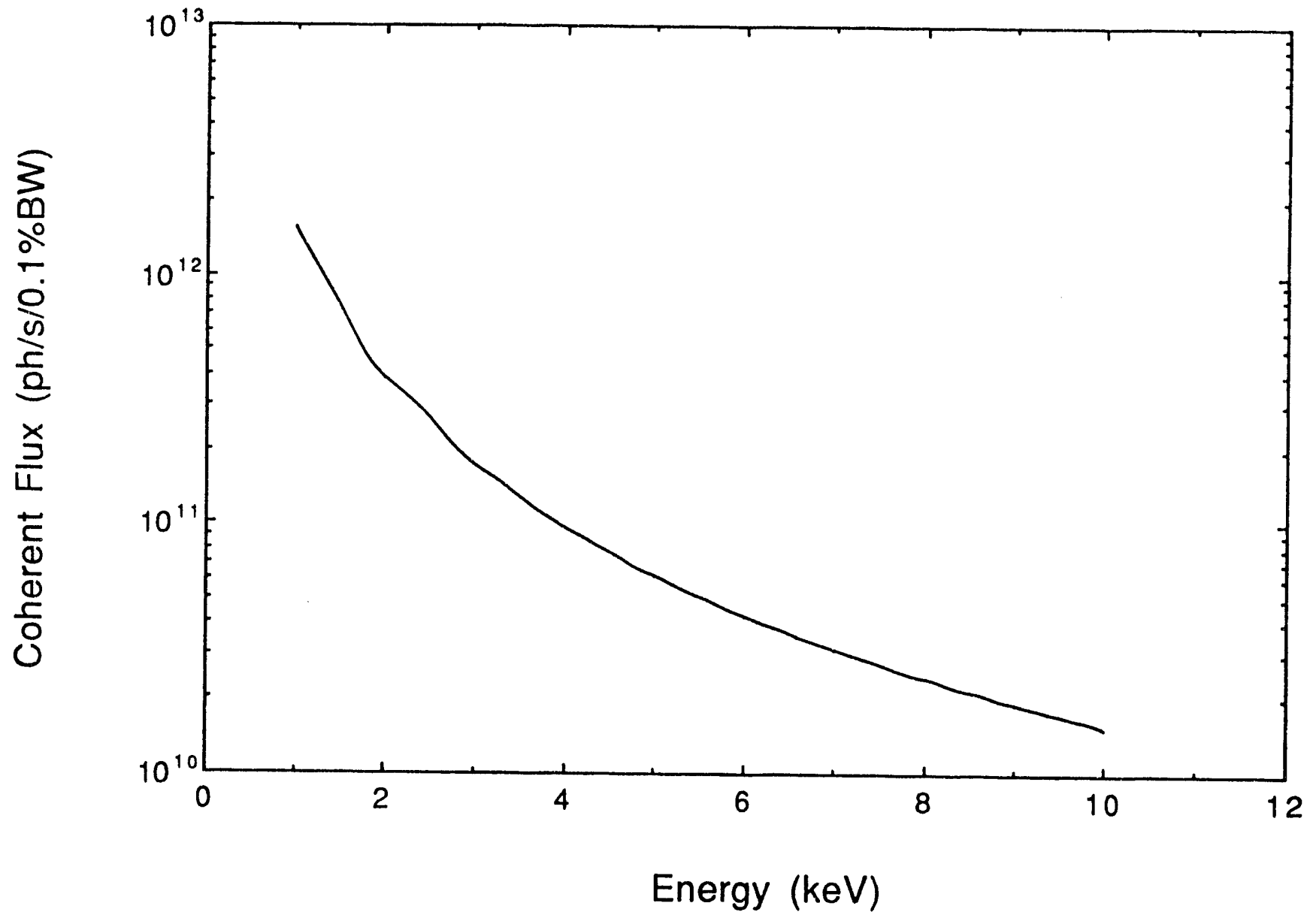

Figure 2.3.15 Coherent flux from a 5-m-long undulator on the APS with a typical brilliance of $10^{18} \mathrm{photons} / \mathrm{sec} / 0.1 \% \mathrm{BW} / \mathrm{mrad}^{2} / \mathrm{mm}^{2}$ over the energy range 
range, the brilliance does not increase quadratically with the number of periods. For the case of Type A Undulators, the segmentation of a 5-m device into two $2.5-\mathrm{m}$ devices reduces the brilliance by only about $13 \% .8$ There are many advantages to using segmented undulators both from the undulator manufacturing concerns and the experimental requirements.

\section{BEAMILINE FroNT ENDS}

\subsection{Introduction}

The front end of a beamline has specific functions. These have been defined for the current phase beamline front ends, and the principal functions are listed below:

- maintain the storage-ring vacuum integrity,

- confine the photon beam power to safeguard all front end and beamline components,

- maintain adequate capability to handle the largest power and power density on front end components,

- ensure personnel safety with triple redundancy and logical control systems during both the commissioning and operating phases,

- provide required information on the angular and spatial position of the photon beam to keep it to a specified stability.

In the current phase construction, 32 front ends will be built; 16 of these will be on the ID beamlines and the remaining 16 will be on the BM beamlines. APS IDs include both wigglers and undulators, and the properties of the radiation produced by these devices will vary. However in the current construction phase, ID front ends are designed to handle the radiation from all the proposed devices that occupy the Type A Sectors.

A layout of the front end components for an ID beamline is shown in Fig. 2.4.1. Starting from the source end, the following components are identified:

Exit Valve - An all metal valve that isolates the front end from the storage ring. Opening this valve is controlled administratively. It is open when the ID is operational.

Photon Beam Position Monitor (photon BPM) - A system capable of detecting the position of the beam both in the $\mathrm{x}$ and $\mathrm{y}$ directions without interfering with the central part of the beam. In combination with a second photon BPM placed 5-7 m downstream, it specifies the precise angular and spatial location of the beam. Photon beam missteering is controlled to within $10 \%$ of its angular and spatial size with a feedback loop to correction magnets in the storage ring that adjust the position and angle of the positron beam. 


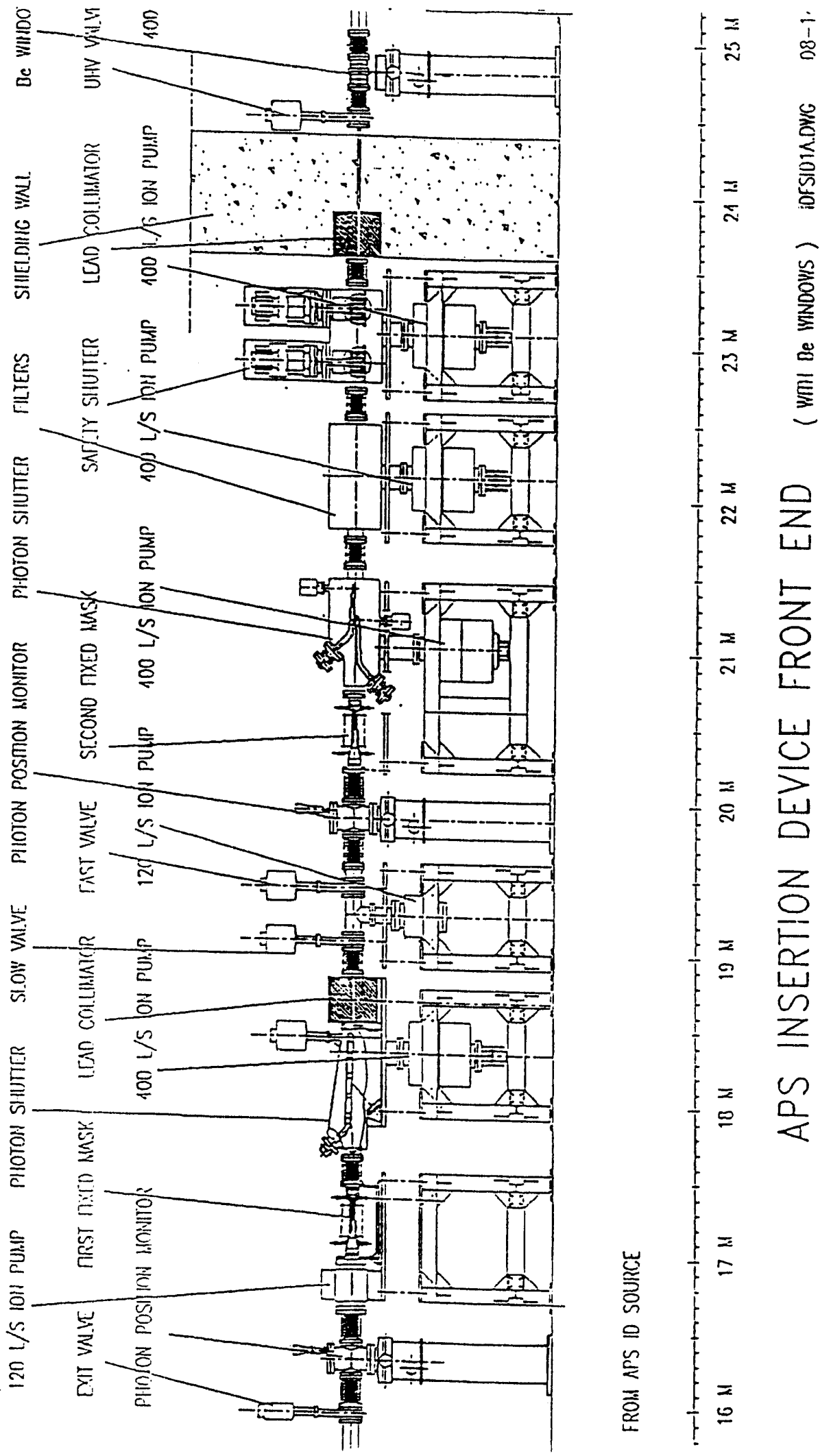

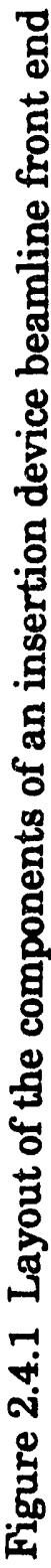


Fixed Mask (Aperture) - A vertical and/or horizontal set of cooled plates that confine the beam to a defined size and admit radiation into downstream components.

Collimators - These components define the line of sight to the source point and admit a cone of radiation. Portions of the beam outside the predefined cone, scattered $\mathrm{x}$-rays, and the bremsstrahlung radiation are absorbed by the collimator body.

Slow Valve - An all-metal, remotely actuated, non-cooled UHV valve that seals to isolate the storage ring vacuum from any vacuum breach in the downstream transport.

Fast Valve - A very fast acting valve, non-cooled, that retards the shock wave progression upstream in case of a vacuum breach in the downstream components.

Filters - Typically, cooled pyrolitic graphite or diamond foils to absorb and dissipate heat from unwanted spectral portions of the photon beam.

Safety Shutters (Injection Stops) - High-Z, metal-based safety blocks, usually in tandem, that absorb bremsstrahlung radiation produced during cold fills of the positron beam into the storage ring.

Window - A vacuum separator that isolates the vacuum of the front end and the storage ring from that of the beamline on the experiment floor. This window (located outside the shield wall) allows the transmission of the photons to the experiment. A differential pumping system has been developed to replace the window when low energy radiation is to be delivered to the experiment.

A detailed logic has been developed with the required redundancy to provide personnel safety in the operation of all the components of the front end. The BM front end has basically the same set of components. The capability to handle the power delivered by various radiation sources is crucial to the final design of all the front end components. Currently, R\&D and testing of prototypes of various front end components are underway to meet the demands of the radiation sources proposed for the Type A Sectors.

\subsection{Front Ends in Type A and Type B Sectors}

A detailed discussion of the angular dependence of the radiation power from the insertion' device sources and bending magnet source forming the Type A Sector can be found in the current phase Design Handbook. The crucial number is the peak power density normal to the surface at a specified distance from the source where the various front end components will be located. The total power from the IDs is equally important in the design of the front end components. Many measures are being implemented in the front end design, first, to reduce the power and, second, to handle the power. In arriving at the engineering designs of the components of the 
front end, extreme care is taken to select the best heat transfer methodology, material properties, stress reduction methodology, etc.

Because of the large power densities involved, front end components such as masks or shutters cannot be placed at normal incidence to the ID radiation beam, regardless of how efficiently they are cooled.

In Tables 2.4.1 and 2.4.2, the power densities and powers delivered by various IDs in Type A and Type B Sectors are presented. Note that the values of power and power densities from IDs on Type B Sectors are comparable to those on Type A Sectors. The only exception to this is the 5-m Undulator A (or double and segmented undulators discussed in Section 3.3.5) planned for Type B Sectors in the APS Instrumentation Initiative. This device delivers twice the peak flux. It is expected that the R\&D in heat transfer technology will lead to front-end components capable of handling the large peak flux from such devices. 
Table 2.4.1

Power and Power Densities Delivered

by Various Insertion Devices in Type A Sectorsa

\begin{tabular}{lcccr}
\hline & $\begin{array}{c}\text { Undulator } \\
\text { A }\end{array}$ & $\begin{array}{c}\text { Undulator } \\
\text { B }\end{array}$ & $\begin{array}{c}\text { Wiggler } \\
\text { A }\end{array}$ & $\begin{array}{c}\text { Wiggler } \\
\text { B }\end{array}$ \\
\hline Device Length (m) & 2.5 & 2.5 & 1.5 & 2.5 \\
Maximum Field (T) & 0.8 & 0.4 & 1.0 & 0.3 \\
Total Power (kW) & 5.0 & 1.25 & 4.6 & 0.7 \\
Peak Power Densityb & 150 & 125 & 26 & 7.8 \\
Peak Flux @ 16 m c & 0.59 & 0.49 & 0.10 & 0.03 \\
Peak Flux @ 24 m c & 0.26 & 0.22 & 0.05 & 0.014 \\
\hline
\end{tabular}

a The current in the storage ring is assumed to be $100 \mathrm{~mA}$.

b Units are $\left(\mathrm{kW} / \mathrm{mrad}^{2}\right)$

c Units are $\left(\mathrm{kW} / \mathrm{mm}^{2}\right)$

Table 2.4.2

Power and Power Densities Delivered

by Various Insertion Devices in Type B Sectors ${ }^{a}$

\begin{tabular}{lcccc}
\hline & $\begin{array}{c}\text { Undulator } \\
\text { A }\end{array}$ & $\begin{array}{l}\text { Elliptic } \\
\text { Wiggler }\end{array}$ & $\begin{array}{c}\text { High Ec } \\
\text { Wiggler }\end{array}$ & $\begin{array}{c}\text { Supercond. } \\
\text { Wiggler }\end{array}$ \\
\hline Device Length (m) & 5.0 & 2.5 & 1.5 & 1.0 \\
Number of Periods & 144 & 16 & 35 & 1 \\
Maximum Field (T) & 0.8 & 1.0 & 1.0 & 3.0 \\
Critical Energy (keV) & - & 32.0 & 58.0 & 98.0 \\
Total Power (kW) & 9.92 & 7.75 & 4.65 & 1.95 \\
Peak Power Densityb & 300 & 41.6 & 91.0 & 7.8 \\
Peak Flux @ 16 m c & 1.17 & 0.16 & 0.36 & 0.03 \\
Peak Flux @ 24 m c & 0.52 & 0.07 & 0.16 & 0.013 \\
\hline
\end{tabular}

a The current in the storage-ring is assumed to be $100 \mathrm{~mA}$.

$\mathrm{b}$ Units are $\left(\mathrm{kW} / \mathrm{mrad}^{2}\right)$

c Units are $\left(\mathrm{kW} / \mathrm{mm}^{2}\right)$ 


\section{BEAMLINES IN THE APS INSTRUMENTATION INITIATIVE}

\subsection{Introduction}

In this section, current plans for building the beamlines in the APS Instrumentation Initiative are presented. It has been pointed out earlier that in the current construction phase the demand for sectors to build beamlines by Collaborative Access Teams (CATs) exceeds the planned development of 16 Type A Sectors. Hence, another four Type A Sectors will be available in the APS Instrumentation Initiative to user groups. These will be allocated to various CATs through the proposal evaluation process that was established for the current construction phase.

Five remaining sectors on the APS will be developed to support special purpose beamlines. These sectors are referred to as Type B Sectors. The research programs on Type B Sector beamlines each has a specific long term goal. Normally, such a program represents a unique and specialized forefront technique or methodology of interest to a group of researchers.

\subsection{Description of Typical Special Purpose Beamlines}

\subsubsection{Three-Dimensional Holographic Imaging Facility}

\subsubsection{Introduction}

The ability to image submicron features with $2-8 \mathrm{keV} x$-rays and focus them to submicron spot sizes opens the door to a wide variety of experi. from high spatial resolution coupled with deep probe penetration. X-rays in this energy range are well suited to imaging microscopic features imbedded in relatively thick objects. Among the various methods capable of providing three-dimensional information about the structure of such specimens, $x$-ray holography stands out as an imaging technique offering both phase and absorption contrast, high transverse resolution, and limited depth resolution from single holographic views. Considerably improved depth resolution is achievable by recording several holograms of the same object from various view angles. The holograms can then be assembled according to the principles of diffraction tomography to increase the effective numerical aperture and, thereby, the spatial resolution. Because hologram formation is a parallel rather than a serial process, the time required to record a hologram is limited solely by the time needed to obtain an acceptable signal-to-noise ratio. Furthermore, by utilizing clustered optics and detectors, multiple holograms of an object can be recorded simultaneously to improve the 3D resolution. This type of aperture synthesis is promising for $\mathrm{x}$-ray microscopy because optics with a large numerical aperture are impractical due to the near unity refractive index for $x$-rays in matter.

\subsubsection{Motivation}

$\mathrm{X}$-rays in the $2-8 \mathrm{keV}$ energy region are potentially valuable for investigating the structure of both organic and inorganic specimens, with a lower absorbed dose and 
through greater thicknesses than is possible with charged particle probes such as electrons. Biological objects are largely composed of low-Z elements that can have significant phase-shifting but relatively weak absorptive effects on the incident $x$ ray beam. Holographic phase contrast is likely to offer the best means to image biological objects with minimal dose $\mathrm{x}$-rays. By contrast, the K-shell absorption edges of many elements in the middle of the periodic table occur in this energy region, provis ling natural absorption contrast for elements such as titanium, chromium, copper, and nickel. A potentially important industrial application of $x$ ray holography is to image interconnects and micro-defects in microelectronic circuits.

\subsubsection{Why at the APS?}

The demand for source brilliance increases rapidly as the imaging resolution is extended to ever smaller dimensions using ever shorter wavelengths. For radiation with a fixed coherence length, the coherent flux and, therefore, the required brilliance scales as the inverse cube of the wavelength. It is for this reason that, before the advent of high brilliance undulator sources in synchrotron storage rings, the exposure times necessary to record $\mathrm{x}$-ray holograms with submicron resolution were impossibly long. The coherent flux $\mathrm{F}_{\mathrm{c}}$ is conservatively given in terms of the source brilliance B by

$$
F_{c}=B(\lambda / 4 \pi)^{2}
$$

The brilliance of the undulator sources planned for the APS exceeds $10^{18}$ photons $/ \mathrm{s} / \mathrm{mm}^{2} / \mathrm{mrad}^{2}$ per $0.1 \%$ bandpass over a sizable range of the $\mathrm{x}$-ray spectrum. At a wavelength of $0.4 \mathrm{~nm}(3.1 \mathrm{keV})$ and a bandpass of $0.1 \%$, the coherent flux is $2 \times 10^{11}$ photons/s. Even with a typical beamline efficiency of a few percent, we can expect there to be sufficient $x$-ray flux to illuminate, for example, a 100-micron-square area with $10^{3}$ coherent photons per 0.1 micron resolution element per second. No other hard x-ray source will compare in brilliance with the Advanced Photon Source for some time to come, making it highly attractive for $\mathrm{x}$ ray holography above $1 \mathrm{keV}$.

\subsubsection{Beamline Design}

The chief aim of an x-ray holography beamline is to maximize the throughput to the experiment while conserving the brilliance of the source. It is also important to provide a suitable degree of tunability, monochromatization, and focusing of the beam insident on the specimen. Absorption and phase contrast can be maximized by tuning the beam energy to features of interest in the specimen being imaged. Sufficient monochromaticity is necessary for formation of the interference fringes that make up the hologram, and concentrating the coherent flux onto the specimen enables the most efficient use of the source brilliance. Finally, the beamline should ideally provide a means of adjusting the degree of coherence of the wave illuminating the experiment to allow the highest obtainable imaging resolution to be traded for shorter exposure times at lower resolution. A monochromaticity of 1000 and a spatial coherence width of $100 \mathrm{~mm}$ at the experiment is adequate for 
many of the holography experiments being considered. Beamline optics suitable for x-ray holography are shown in Fig. 2.5.1. The conceptual beamline, which uses the Fresnel configuration, is shown in Fig. 2.5.2.

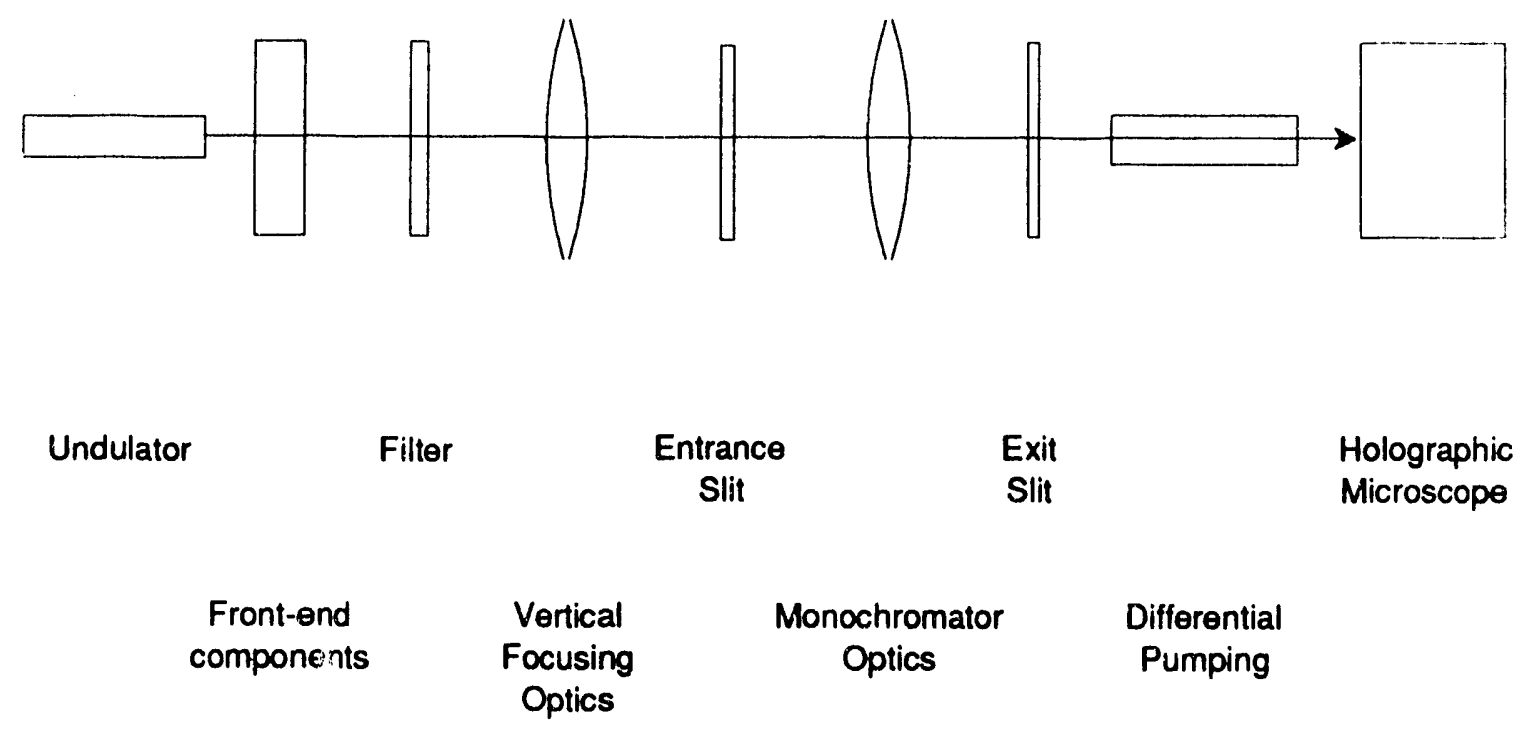

Fig. 2.5.1 Principle of $x$-ray holographic imaging at the APS

\subsubsection{Radiation Damage to the Specimen}

Careful assessment of the risks of specimen radiation damage is crucial to the practical success of $x$-ray holographic microscopy. It is not yet clear, particularly in the case of biological samples, what limits pertain to the obtainable imaging contrast, and, therefore, what dose can be given before an unacceptable level of damage is sustained to the sample at a given resolution. Further study is required to determine the damage penalty incurred when the imaging resolution is extended from two to three dimensions.

\subsubsection{Very High Energy X-ray Scattering Facility}

\subsubsection{Introduction}

The ability to provide a high intensity beam of photons from $50 \mathrm{keV}$ to $1 \mathrm{MeV}$ presents a novel opportunity for experiments in this largely unexplored energy range. A variety of elastic and inelastic scattering experiments will benefit from the use of very high energy $x$-rays.

The energy transfer of Compton-scattered photons increases with energy and, for the very high x-ray energies being considered here, would allow the impulse approximation to be used with impunity. For high-Z elements, the ratio of the incoherent to coherent cross-sections is greatly increased in this energy range 


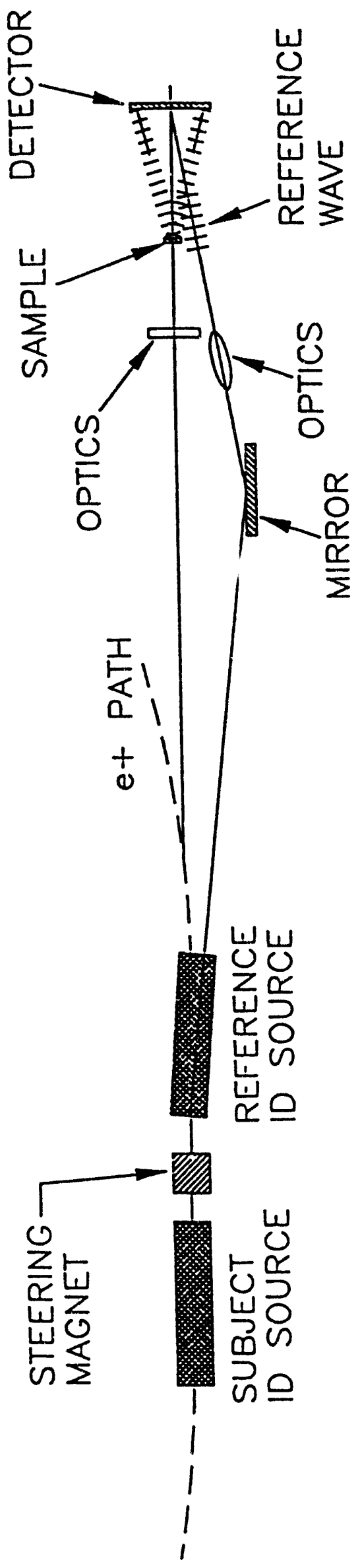

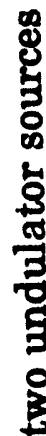

照

롬

\&

ஸू. 
(see Fig. 2.5.3), making possible the study of Compton scattering from heavy elements. Additionally, as the incident energy approaches $\mathrm{mc}^{2}$, spin-dependent Compton effects become more important and can be studied.

Recently, Compton-scattering experiments have been performed in which the scattered photons and electrons were measured in coincidence (the so-called $\gamma e \gamma$ experiments). The information obtained from a $\gamma$-e experiment allows the direct determination of the electron momentum density of a solid material. In these measurements, multiple scattering of the electrons has proved to be troublesome and, therefore, very thin samples have had to be used. A very high energy incident $\mathrm{x}$-ray beam reduces this problem considerably.

It is now well established that $\mathrm{x}$-rays above $100 \mathrm{keV}$ are very useful for elastic scattering studies. Characterizations of thick single crystals have been made using triple-axis diffractometry on such diverse materials as perfect $\mathrm{Si}$ and $\mathrm{Ge}$, annealed oxygen-doped $\mathrm{Si}, \mathrm{Si}-\mathrm{Ge}$ gradient crystals, mosaic $\mathrm{Be}$, and Ni-based superalloys. It should also be possible to study systems (such as buried interfaces) in which photon absorption is a prohibitive problem at regular x-ray energies. Also, because the dispersion corrections are very small for most materials at these energies, it is possible to make very accurate structure factor measurements.

With high energy $x$-rays, it will be possible to study the absorption profiles over the whole range of elements. The $K$ edges of the very heavy elements will be accessible directly, while the $L$ and $M$ edges of heavy elements and the $K$ edges of lighter elements can be explored through inelastically scattered photons. In the heavy elements, the binding energy of the $\mathrm{K}$ electrons approaches the rest mass of an electron, and relativistic effects begin to manifest themselves.

It will be possible to carry out experiments with nuclear excitations. The energy produced by the proposed device will allow many states to be reached with much greater photon flux than is possible with current sources. Direct excitation will allow studies of nuclear resonance scattering. In addition, very narrow (in energy) states that are not accessible with direct excitation may be pumped via a higher lying broader state. This will allow production of photons with an extremely narrow energy spread at a variety of energies.

Many other interesting experimental possibilities exist covering a variety of scientific fields. A few are listed here. 1) Diffraction with very large momentum transfer. Radiation of $1.50 \mathrm{keV}$ has been used to measure the $(20200)$ reflection from $\mathrm{Si}$. At such high order reflections, the zero point motion of the atoms is greatly enhanced, and fundamental aspects of thermal motion may be studied. 2) Tomography on very dense materials. The study of high- $\mathrm{Z}$ materials through radiographic methods has been impossible due to the poor penetration of normal energy $x$-rays in these materials. The use of $x$-ray energies in excess of $100 \mathrm{keV}$ would allow examination of components made from materials such as tungsten, uranium, and tantalum. 3) Testing of $\psi$-ray optics used for astronomy and other $\gamma$-ray applications. The flux for the proposed beamline will allow devices designed to 


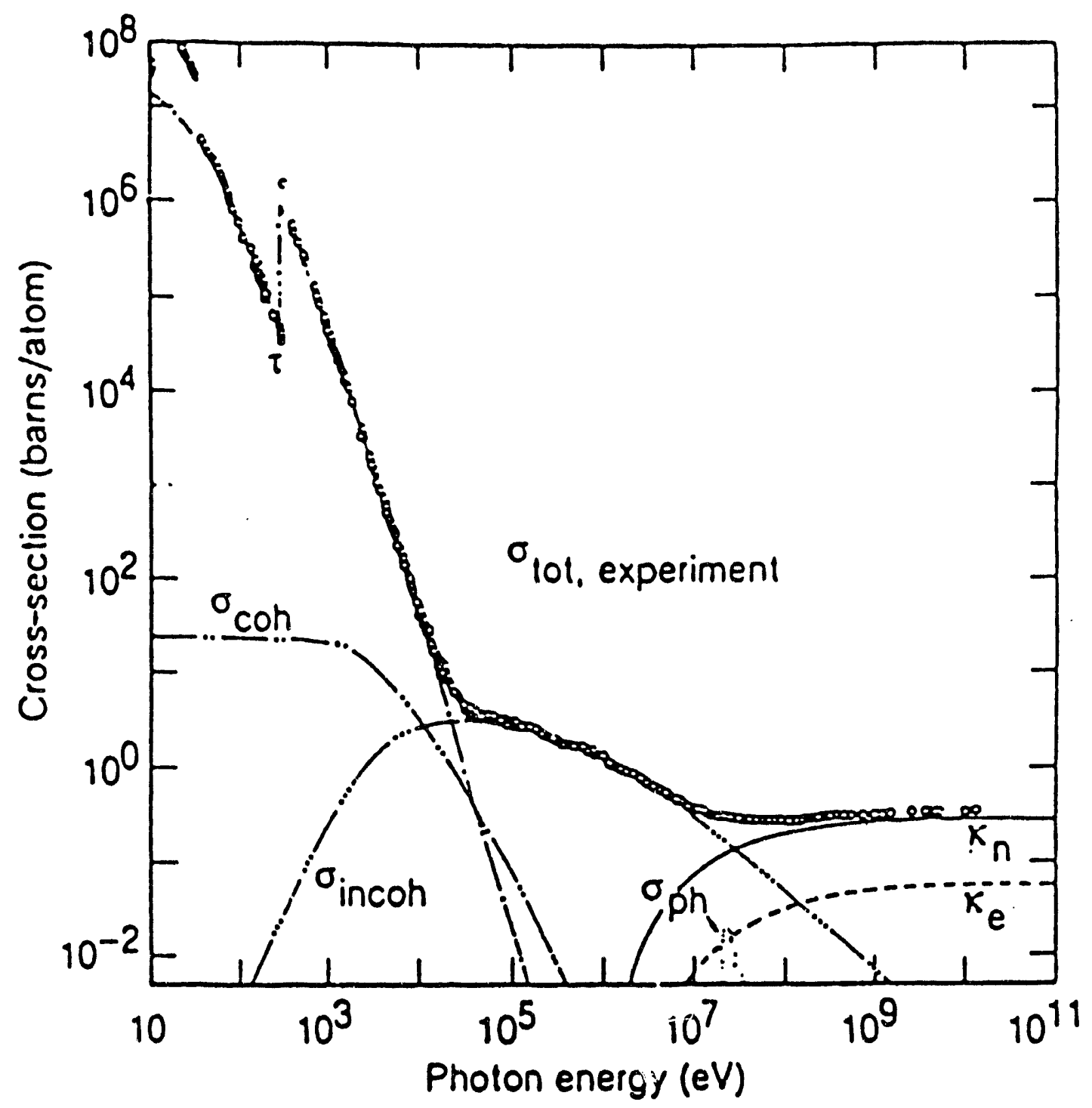

Fig. 2.5.3 Total photon cross section $\sigma$ tot in carbon, as a function of energy, showing the contributions of different processes: $\tau$ : atomic photoeffect (electron ejection, photon absorption); $\sigma_{\text {coh }}$ : coherent scattering (Rayleigh scattering atom neither ionized nor excited); $\sigma$ incoh: incoherent scattering (Compton scattering off an electron); $\kappa_{\mathrm{n}}$ : pair production, nuclear field; $\kappa_{\mathbf{e}}$ : pair production, electron field; $\sigma_{\text {ph }}$ : photonuclear absorption (nuclear absorption, usually followed by emission of a neutron or other particle) ${ }^{9}$ 
detect $\boldsymbol{\gamma}$-rays from very weak sources to be tested and characterized before being placed in operation.

\subsubsection{Conceptual Layout of the Beamline}

A schematic of the beamline is shown in Fig. 2.5.4. After the wiggler, filters will be employed to remove the lower energy photons and reduce the heat load on subsequent optics. The first station will be used primarily for elastic scattering experiments and will contain a high-precision, triple-axis Laue diffractometer. The diffractometer will also serve as a monochromator for the second station. It will be primarily used for inelastic scattering studies and will contain the necessary equipment for various types of Compton-scattering experiments.

This beamline is envisioned initially with a 3-Tesla superconducting wavelength shifter as the insertion device. With the APS operating at $7 \mathrm{GeV}$, such a device would produce a critical energy of $98 \mathrm{keV}$ (see Section 3.3.4 - Wigglers with Large Critical Energies). This would provide ample flux at $x$-ray energies up to and beyond $0.5 \mathrm{MeV}(500 \mathrm{keV})$. However, even higher field insertion de vices could be explored. For instance, it is possible to construct a 7.5-T superconducting wiggler. If used on the 7-GeV APS storage ring, it would produce $x$-rays with a critical energy of $244 \mathrm{keV}$. The energy spectrum for such a device is shown in Fig. 2.5.5.

A facility for $50 \mathrm{keV}$ to $1 \mathrm{MeV}$ photons presents several requirements that can be better accommodated by a dedicated beamline. The optics required for the manipulation of $x$-rays in this energy range differ considerably from those used for normal x-ray energies. This beamline will also require additional shielding compared to that required for other APS beamlines. It will not be possible to install the proposed wiggler onto an existing beamline without essentially rebuilding the hutches and transport, and, hence, it is unlikely that an existing program will upgrade to include such an insertion device. Also, the lack of previous experience with photons in this energy range by most current synchrotron users and the rather broad range of science for which it should be designed make it unlikely that a single outside CAT would built such a beamline. A dedicated beamline run by the APS will allow the opportunity for scientists from several fields to conduct experiments that complement their current work while developing the expertise need $d$ to fully utilize photons in this energy range.

\subsubsection{Complete Polarization Facility}

\subsubsection{Introduction}

In recent years, the use of polarization properties of synchrotron radiation has become increasingly important to many researchers in both fundamental and applied sciences. By use of polarized $x$-rays, a new era in studying magnetic materials has begun. Spin structures of bulk magnetic materials, multi-layer magnetic systems, and magnetic interfaces can be studied with linearly polarized xrays. Furthermore, momentum distribution of magnetic electrons in ferro- and ferri-magnetic materials can be determined by using circularly polarized photons in inelastic magnetic scattering experiments. The advantage of using polarized $\mathrm{x}$-rays 


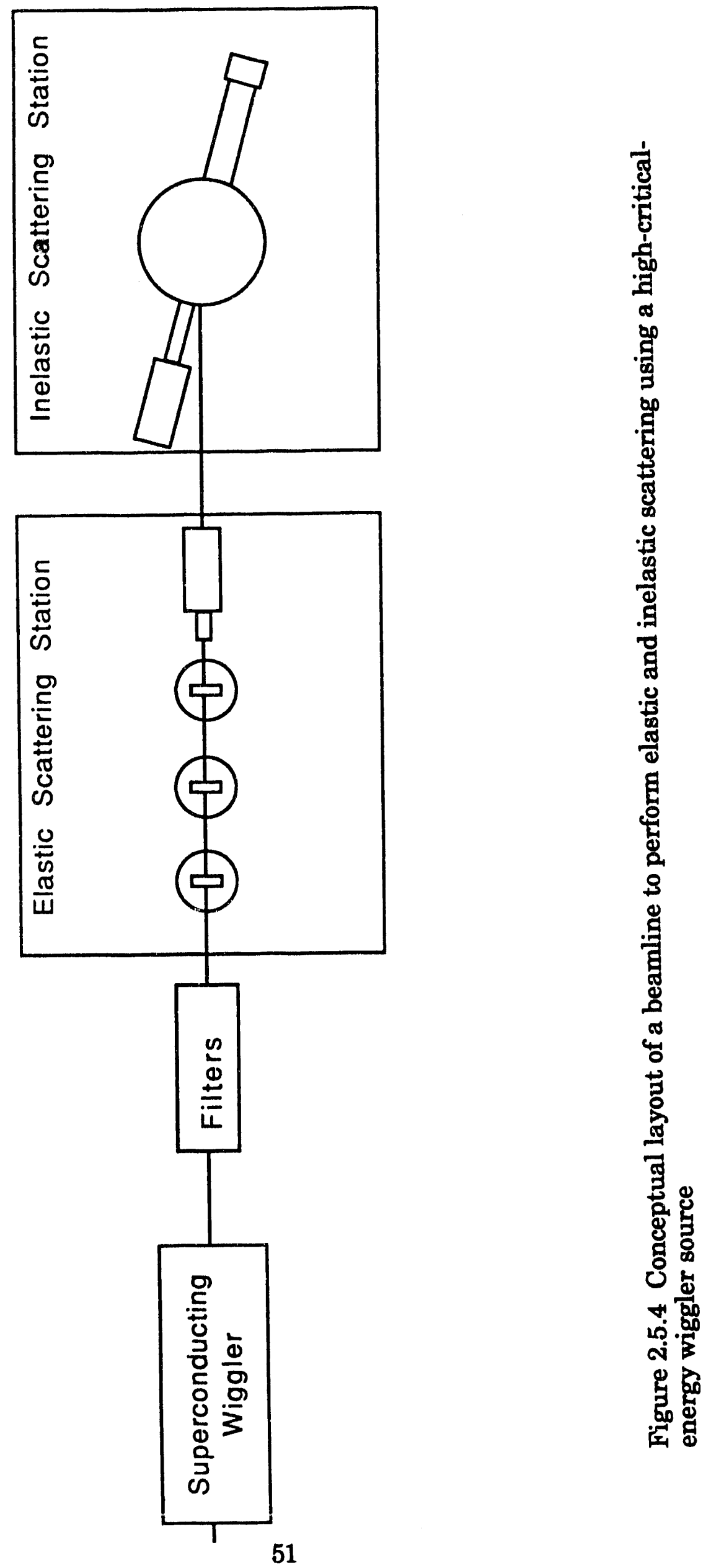




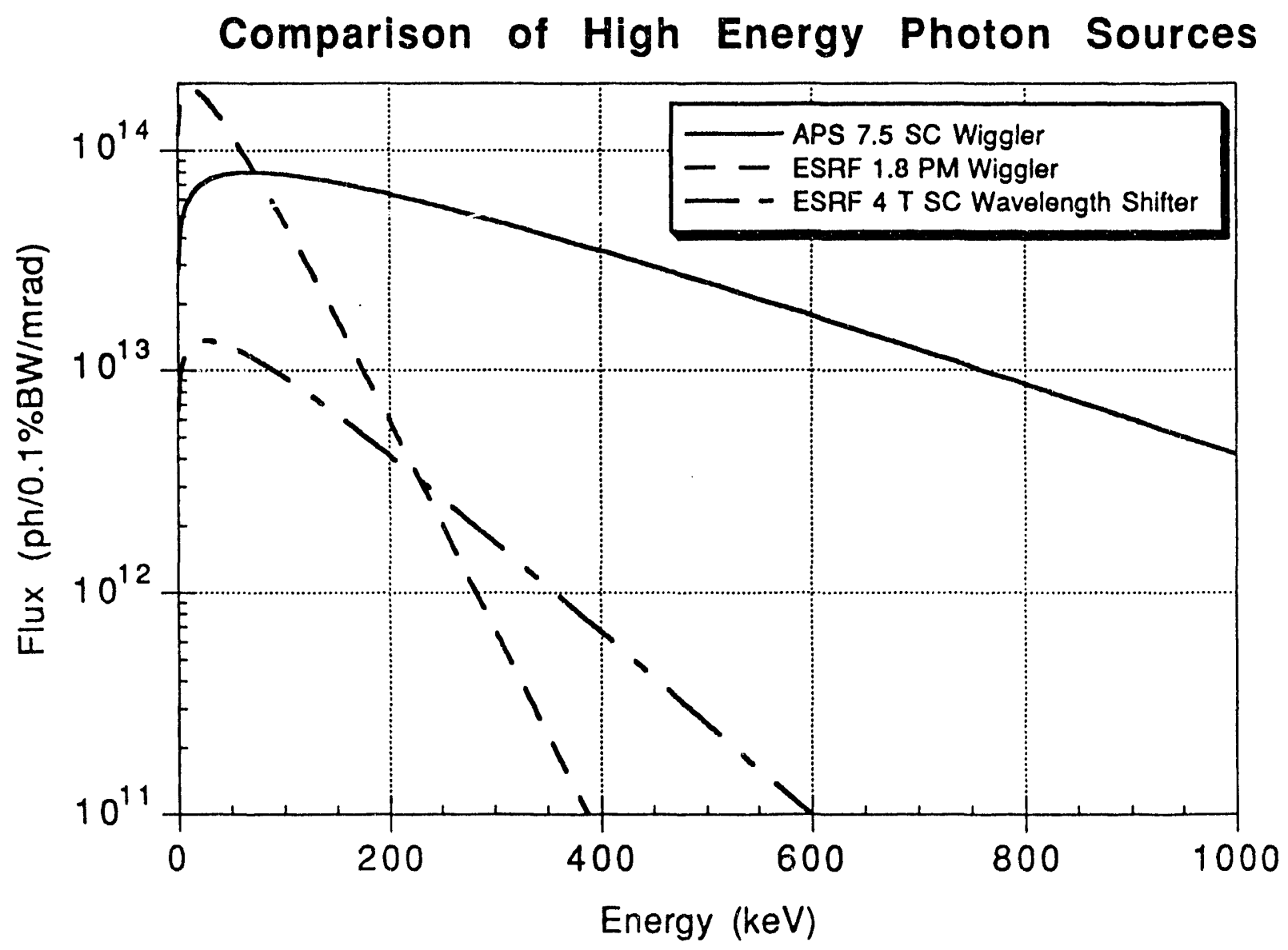

Fig. 2.5.5 Flux from a 7.5 Tesla superconducting wiggler on the APS as a function of energy. Comparison is made with a 1.8 Tesla permanent magnet wiggler and a 4 Tesla superconducting wiggler planned for ESRF 
over polarized neutrons in many magnetic scattering experiments is that polarized $x$-rays can resolve orbital magnetization from spin magnetization. 10,11 Smaller samples can also be more easily probed due to the intrinsic brightness of the source. Both elastic and inelastic magnetic scattering experiments require high brilliance, high momentum and energy resolution (on the order of $10^{-4}$ ), and a wide energy range. In addition, it will be possible to extend visible-light techniques, such as the Kerr effect, optical birefringence, and Faraday rotation experiments, to the x-ray regime.

\subsubsection{Basis for the Beamline Concept}

The radiation emitted from bending magnets and conventional insertion devices is highly linearly polarized in the plane of the particle's orbit. Elliptically polarized $x$-rays can be also obtained by going off-axis with, however, a considerable loss of flux. The application of polarized x-rays has been largely motivated by recently developed techniques that use perfect single crystals as polarizers and analyzers. The proposed Instrumentation Initiative beamline of this facility will house specialized insertion devices, such as helical undulators 5 and/or elliptical multipole wigglers (See Section 3.3.3 - Circular Polarization Sources) that will be able to produce a large flux of highly polarized $x$-ray photons. It is envisioned that the state of polarization of the emitted radiation from these specialized insertion devices be rapidly tunable, from say right-handed circularly polarized x-rays to left-handed circularly polarized x-rays. However, production and modulation of polarized x-ray beams via specialized insertion devices does not solve the entire problem of delivering highly polarized beams into a sample. Polarization-preserving monochromators must be designed, fabricated, and tested. These monochromators, particularly those designed for circularly polarized radiation, will in all likelihood be significantly different in design and considerably more complex than a standard double-crystal monochromator. 12,13 The combination of specialized insertion devices and polarization-preserving monochromators dictates that this program be on a dedicated beamline for polarized x-ray studies.

In addition to the specialized insertion devices and monochromating system required for this beamline, other specialized instrumentation would be required to perform state-of-the-art polarization studies (Fig. 2.5.6). Typical examples would include high-field, large-gap electromagnets for applying external magnetic fields to orient or align magnetic systems, cryostats and furnaces (compatible with the aligning magnet system! for studying the temperature dependence of magnetic systems, and $x$-ray optical components that would allow for the complete polarization analysis of the incident and scattered $x$-ray beams. 14

\subsubsection{Sub-Nanosecond Temporal Resolution Beamline Facility}

\subsubsection{Introduction}

The unique high-brilliance and short-pulse characteristics of the APS and the development of ultra-short-pulse lasers will provide the opportunity to add a temporal dimension with unprecedented resolution to $\mathrm{x}$-ray studies. The high temporal resolution is obtained by using the pulsed photon sources in a pump-probe 


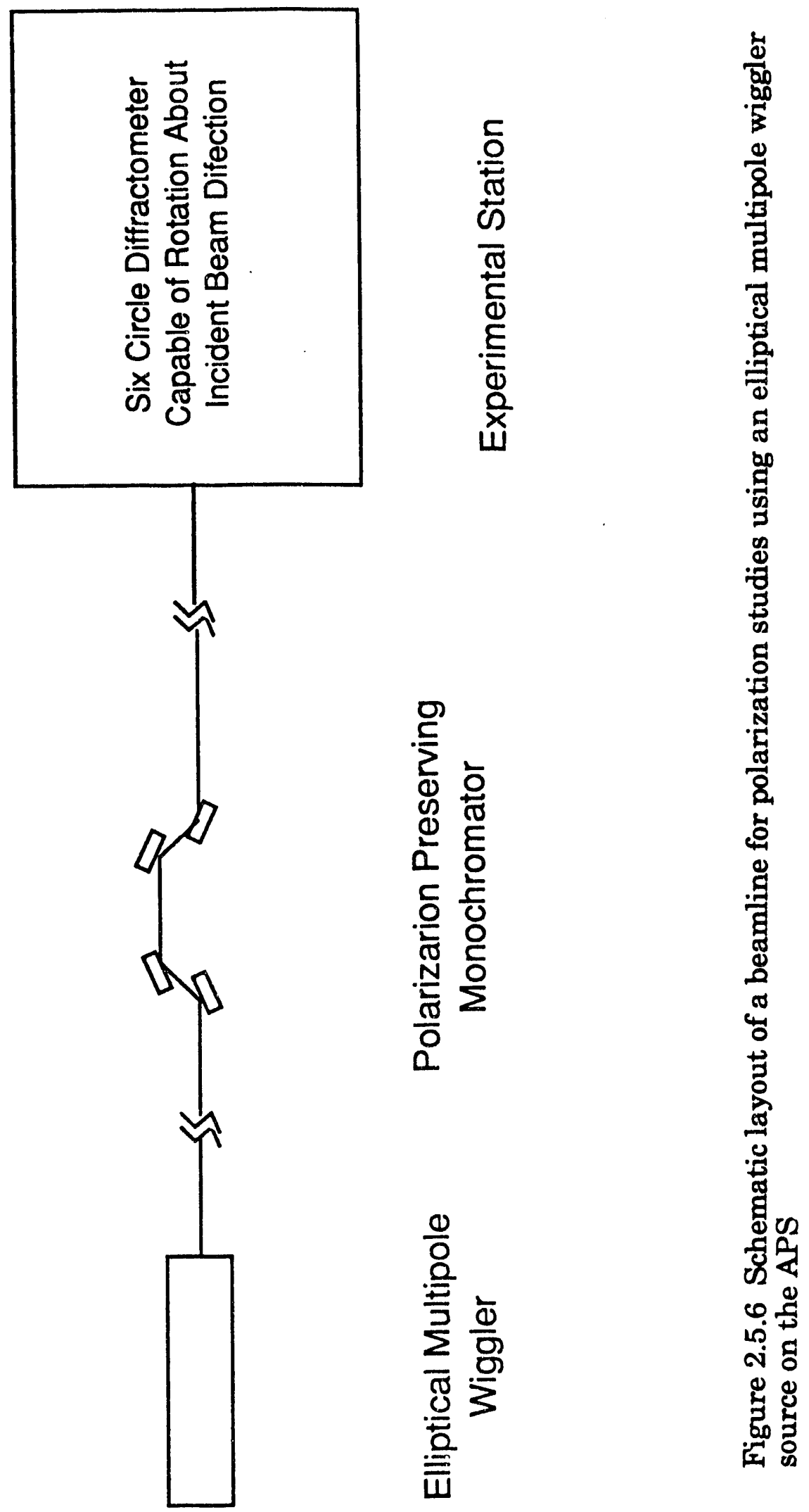


combination. In the pump-probe technique, one of the sources is used to initiate transient phenomena while the other source probes the dynamic process as a function of the relative pulse synchronization. In a pump-probe measurement, the temporal resolution is determined by the pulse duration. Sub-nanosecond resolution is achievable at the APS with its $\sim 100$ picosecond x-ray pulses used with a short-pulse laser (or other pulsed source) with pulses as short as 100 femtoseconds. The pump-probe technique can be applied to study any dynamical process in which transient phenomena (physical, chemical, or biological in nature) are either initiated or measured with $\mathrm{x}$-rays.

Detector and electronic readout times limit the temporal resolution of traditional measurements, 15 while the resolution of the pump-probe technique is determined by the pulse durations. 16 This permits the resolution to be taken from electronic time scales, typically on the order of milliseconds, to short photon-pulse durations in tire pico- to femtnsecond range. With the addition of the time dimension to measurements, the need to increase data collection efficiency becomes more acute. The data collection throughput can be increased by the use of position sensitive detectors (PSD) with fast (fast on electronic time scales) readouts. Thus, PSDs will be used to enhance pump-probe data collection but not limit its temporal resolution.

The pulse durations limit the temporal resolution in the pump-probe technique, and the APS is a unique high-brilliance, short-pulse $x$-ray source. While shorter $x$ ray pulse bursts are obtainable from laser produced plasma emissions, these sources are limited in both brilliance and total flux. A state-of-the-art laser plasma x-ray source 17 can have $x$-ray bursts that are as short as 5-10 picoseconds but their timeaveraged fluxes are many orders of magnitude smaller than those expected from APS sources. Plasma sources also lack the excellent collimation of APS synchrotron radiation and ID emissions. The plasma-generated radiation is typically emitted into $2 \pi$ steradians and even relatively efficient spectrometers 18 are able to collect only a fraction of the radiation. The APS will be the most brilliant $\mathrm{x}$-ray source available, and the pulse duration of the APS compares favorably with other synchrotron sources (see Table 2.5.1).

Table 2.5.1

Temporal Properties of Synchrotron Sources

\begin{tabular}{lrrrr}
\hline & APS & ESRF & SPring-8 & NSLS \\
\hline Bunch Length & 27 & 45 & 28.5 & $: 0$ \\
Orbital Period (ns) & 3683 & 2861 & 4787 & 568 \\
\hline
\end{tabular}




\subsubsection{Beamline Concepts}

In a typical experiment, shown schematically in Fig. 2.5.7, the relative timing of the laser and x-ray pulses will be set by a optical delay line; a structural phase transition will be initiated thermally by a laser pulse, and the diffraction pattern will be measured with an $\mathrm{x}$-ray pulse. This process will be iterated to obtain the diffraction patterns. Thus, structural information about the sample is obtained as a function of the time relative to the heat pulse that initiated the transition.

In addition to the standard beamline x-ray equipment, the experimental station will be equipped with high intensity, short-pulse lasers and the means to accurately control the relative timing of the $x$-ray and laser pulses. A short-pulse-mode locked titanium:sapphire laser and short-pulse-excimer laser can provide pulses of a few hundred femtoseconds and have light from the deep UV through the visible and into the IR ( 250 $\mathrm{nm}$ to $1000 \mathrm{~nm})$. These lasers will provide peak intensities in excess of $100 \mathrm{~kW}$ and can be amplified if higher peak intensities are required.

The sub-nanosecond beamline will be designed to be flexible enough to accommodate a variety of other experimental setups. For example, a dynamical process could be pumped with synchrotron radiation (e.g., creating core vacancies) and probed with the x-ray emission of a short-pulsed laser-produced plasma (see Fig. 2.5.7). (Though these plasma emissions have low intensities, they have emissions from soft $x$-rays to $1 \mathrm{MeV}$ x-rays. ${ }^{19}$ ) Or using a small angle x-ray diffractometer, the dynamics of large molecular systems could be studied. Another interesting possibility for the APS is a beamline configuration with two different IDs on one sector (see Section 3.3.5 - Double Undulator Sources on a Single Straight Section). The two unique spectral outputs could be used for x-ray/x-ray pump-probe when used with an $x$-ray delay line in place of an optical delay line (see Fig. 2.5.8).

The pump-probe technique uses the short pulsed nature of the synchrotron in providing high temporal resolution. The resolution will often be limited by the pulse duration. Even higher resolution might be obtainable if it is possible to run the APS storage ring with shorter bunch lengths, and the sub-nanosecond beamline facilities will be designed to exploit this possibility.

\subsubsection{Facility for Coherence and Interference Techniques}

\subsubsection{Introduction}

The capability of studying both the amplitude and the phase of a signal wave by detecting its interference with a mutually coherent wave front offers many advantages over detection of the signal intensity. 20 For example, not only the spatial distribution of a scattering source can be determined in a holography experiment, but also the detectability of a weak signal can be increased by several orders of magnitude through using its interference with a strong reference beam. Time-resolved speckle diffraction can be used for studying dynamical and kinematic processes. 21 Those advantages have been effectively used in the optical spectral region. In recent years, some preliminary experiments utilizing those properties in the $x$-ray spectral region have been demonstrated. $20,22,23,24,25$ The high 
High Temporal Resolution Pump-Probe Beamline

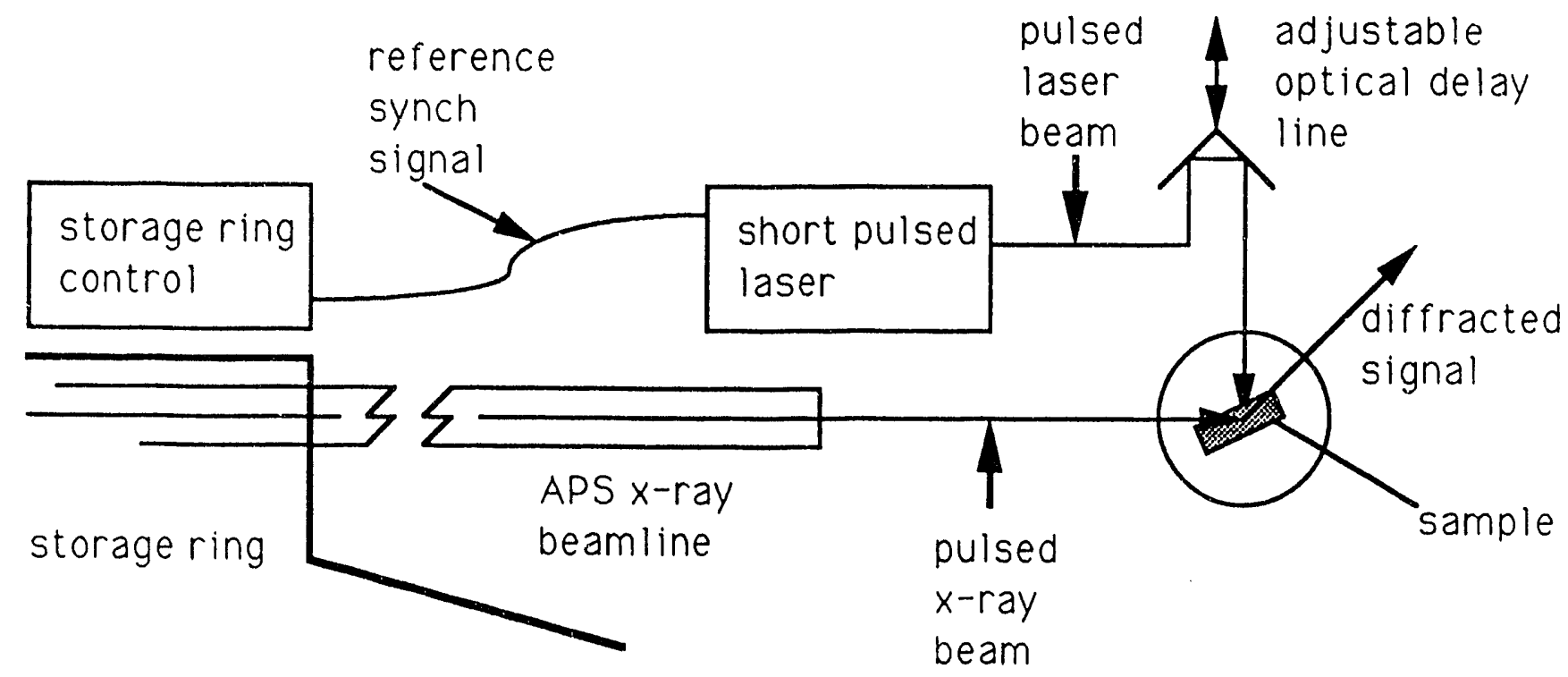

Fig. 2.5.7 Schematic layout of beamline requirements for a high temporal resolution pump-probe beamline for the APS 


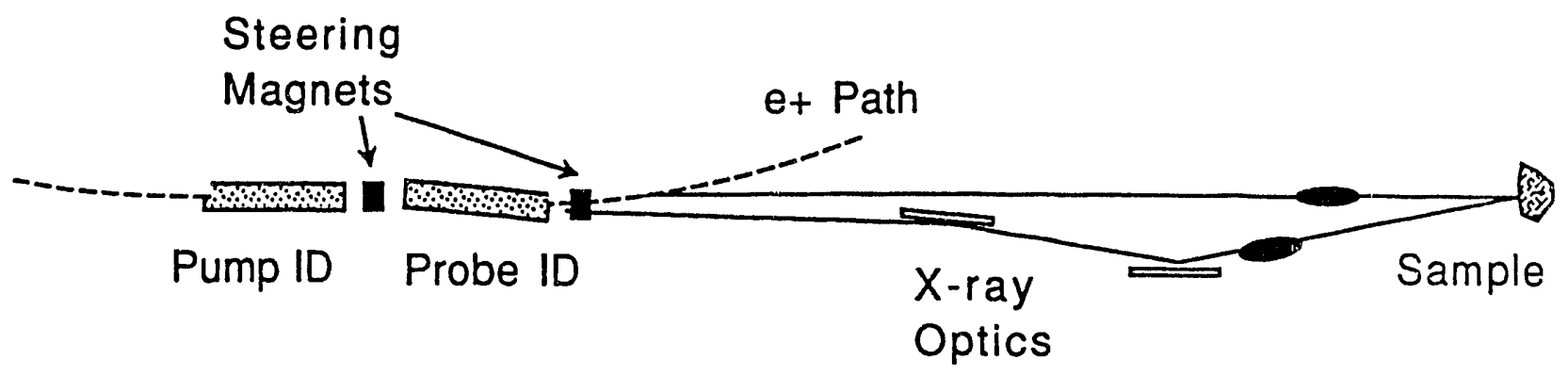

Fig. 2.5.8 Conceptual layout of an APS Instrumentation Initiative beamline for performing temporal studies using two undulators as radiation sources 
brilliance and coherent flux in the hard $\mathrm{x}$-ray region provided by the APS opens up excellent opportunities for exploiting those advantages in the x-ray spectral region. 26,27 Concurrently, some of the stringent requirements in the fabrication of necessary coherent $x$-ray optics have been overcome through rapid advances in the last decade in microfabrication technology, high precision surface finishing, crystal growth, controlled layer deposition, and precision metrology. 28 The dependence of interference on polarization may also be used to enhance or suppress certain polarization components of the signal by adjusting the polarization of the reference beam.

\subsubsection{Purpose}

Unique capabilities that will be provided by coherence- and interference-based techniques include: (1) phase contrast or signal enhancement, which can be used to increase the signal-to-noise ratio in imaging and scattering techniques; (2) study of the spatial distribution of a scattering source, which can be used for Fourier transform imaging of surfaces, 3D holography imaging, and speckle diffraction; and (3) study of spectral properties of a radiation source, which can be used for source property characterization and Fourier transform spectroscopy. The proposed beamlines will focus on the development of techniques that will make effective utilization of the three capabilities discussed above. This development will be imperative in order to take full advantage of the high coherent $\mathrm{x}$-ray flux expected from third generation synchrotron $x$-ray sources. In addition, the findings of the proposed program may have significant impact on the future development of coherent x-ray sources.

The ability to control the coherent volume according to different experiments while preserving the source brilliance is the main design goal of the beamline optical components. Key optical components in a conceptual design of a beamline are shown in Fig. 2.5.9. The phase space aperture will be used for controlling the phase space of the beam. A spatial coherence length of 100 microns at the sample plane will be achieved. The beamline is split into two branches using a deflection mirror, with one serving for $\mathrm{x}$-ray energies less than $4 \mathrm{keV}$ and the other serving for $\mathrm{x}$-ray energies greater than $4 \mathrm{keV}$. Monochromators with resolving power ranging from 100 to about $10^{6}$ will be developed for the high energy branch. For the low energy branch, monochromators of adjustable resolving power of 100-1000 will be necessary. Several optical components inside experimental stations, such as diffraction-limited focusing optics, beam splitters, and phase shifters, are essential components for various techniques. 


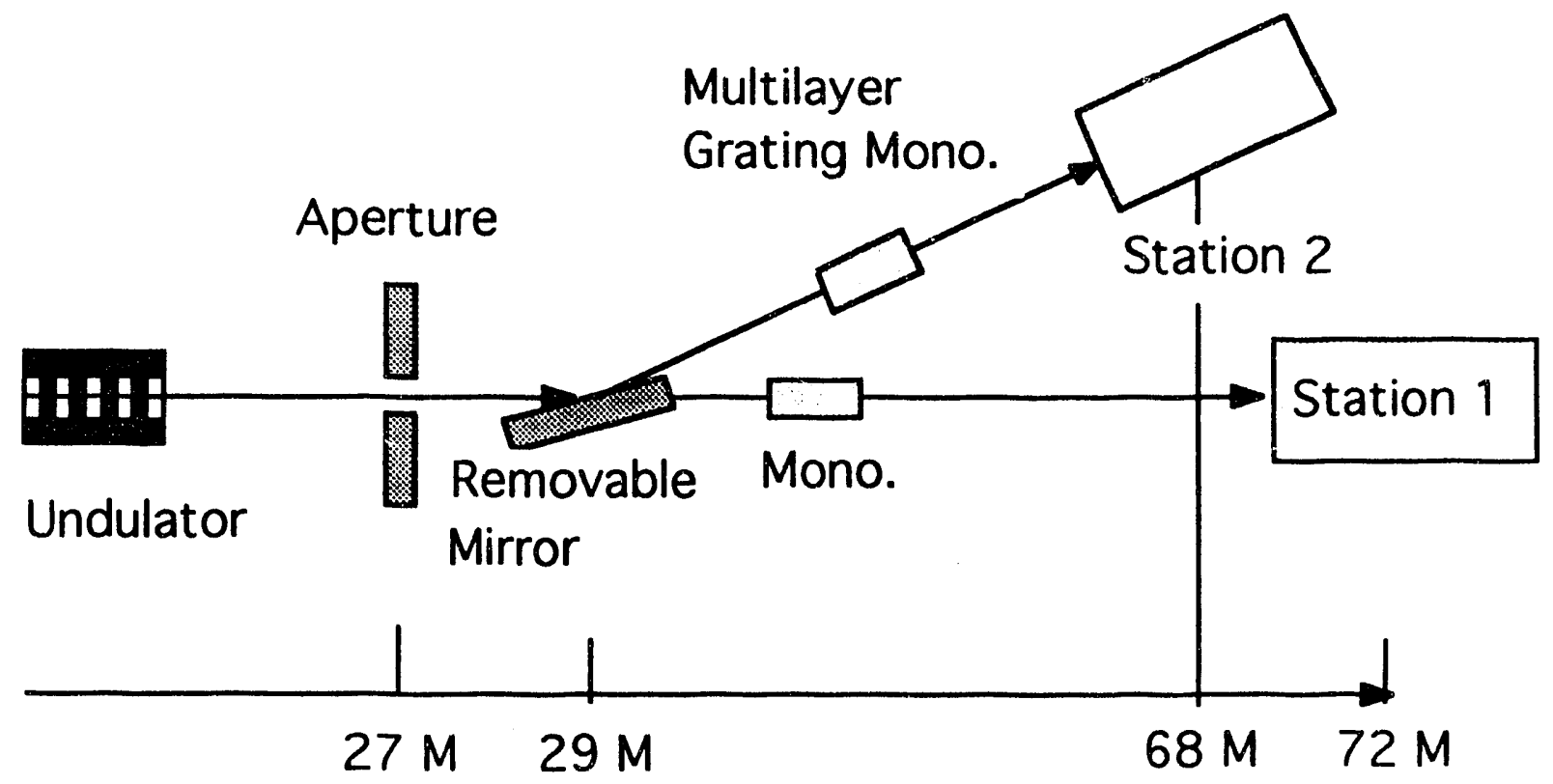

Fig. 2.5.9 Layout of a beamline for performing coherence and interference studies in the APS Instrumentation Initiative (Mono. = monochromator)

\subsubsection{Static and Dynamic Small Angle X-ray Scattering Facility}

\subsubsection{Introduction}

Small angle $\mathrm{x}$-ray scattering (SAXS) is a powerful tool for structural analysis of a wide variety of systems and materials. Typical length scales that can be probed by conventional SAXS range from $10 \AA$ to $5000 \AA$, which makes SAXS suitable for the study of biological macromolecules (e.g., enzymes and other proteins), polymers in solution and solids, and synthetic serriconductors produced by molecular-beam epitaxy. Recently, structures with a scale larger than $10,000 \AA$ have attracted special interest in applied research. For example, modulated metallic alloys and sols and gels may have significant technological implications in the near future. SAXS may be also used as a complementary technique to electron microscopy and quasi-elastic light scattering in which complicated effects due to absorption and multiple scattering often produce inconsistent results. This is particularly applicable to sub-micron polystyrene spheres that are widely used ror calibration purposes in both light and electron microscopy and in sedimentation and aerosol studies.

With current synchrotron sources, the most successful studies have been in the domain of static structures. The application of SAXS to dynamical processes has been limited to a very few systems in which conformal changes do not destroy the crystal lattice. This means that the majority of macromolecular systems must be studied in solution and/or in situ, which, in turn, requires a new generation highbrilliance x-ray source. A five-meter version of Undulator A is ideally suited for such experiments. Furthermore, an $x$-ray source of high brilliance combined with 
optimized point-focusing will enable static structural studies of small volume samples and very dilute solutions. This may be particularly attractive in structural biology where genetic engineering techniques ofteri produce small amounts of rare proteins. Another possible application is in the synthesis of ultra-strong fibers (i.e., Kevlar) where structural information is essential.

SAXS experiments offer the possibility of studying new aspects of dissipative structures that are formed far from equilibrium. Spatially periodic structures that have been observed in chemical-reaction-diffusion systems ${ }^{29}$ are excellent candidates for the study of dynamical processes.

\subsubsection{Beamline Concepts}

The proposed facility to perform static and dynamic SAXS studies is a perfect example of an Instrumentation Initiative special purpose beamline (see Fig. 2.5.10).

One of the instruments that has been widely used for SAXS experiments was developed by Bonse and Hart. 30 The Bonse-Hart (BH) camera is a multiple diffraction diffractometer that utilizes two grooved crystals with the sample placed between the two crystals. Recently, it has been demonstrated 31 that a BH camera can be adapted for operation at high photon energies. The energy chosen was 50 $\mathrm{keV}$ and was limited by the available source. New sources of high-energy photons, like the APS, will make such an apparatus invaluable for the study of highly absorbing samples or samples in difficult environments such as ovens or highpressure cells.

To fully utilize the unique capabilities of the APS to provide a high flux of photons in a wide energy range for SAXS experiments, a dedicated facility should be built. This facility would provide instrumentation and technical expertise for users to set up and successfi ${ }^{-1 y}$ perform SAXS experiments. Because the optics and experimental instrumentation are sufficiently sophisticated and different than those present on a more standard beamline, a dedicated beamline and facility is warranted.

The beamline of this facility should operate at various photon energies depending on the particular sample to be strdied. For most SAXS experiments, very high-resolution is generally not crucial. However, for well-ordered samples, such as membranes, a reasonable resolution of $\Delta E / E \approx 10^{-3}$ is required, which can be obtained by standard double-crystal monochromators. The primary focusing optics consist of two mirrors (Kirkpatrick-Baez). The sample stage will have a BH camera with removable grooved crystals. The facility will provide crystals in the energy range of interest to the user. In addition, to accomplish focused beam sizes in the range of $100 \mu \mathrm{m}$, we propose to use Fresnel zone plates. Specially designed high-framing-rate charge coupled detectors (CCDs), or perhaps (in the future) pixel array detectors (PADs), would be available at this beamline to facilitate live-time studies of dynamic systems. 


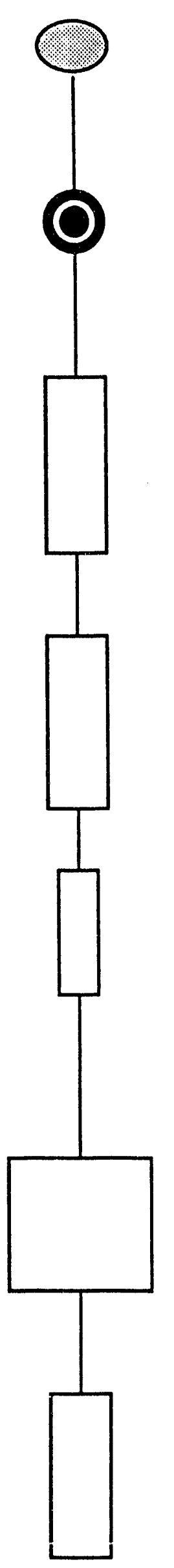

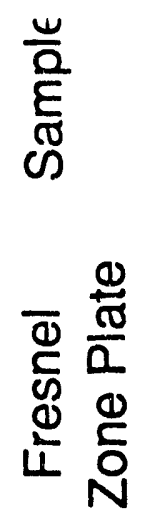

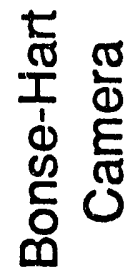

告

옳

엉 흔

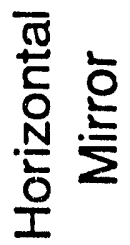

客 ธิ

ㄹ.

ส.

可

․․․

홍 웅

过

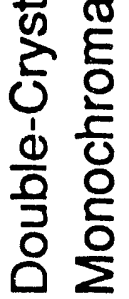

을 훌

$\frac{5}{\frac{0}{0}}$ 


\subsubsection{Ultra-High-Pressure and U1tra-High-Temperature Facility}

\subsubsection{Introduction}

The study of the structural and electronic properties of matter under extreme conditions (pressure and temperature) has been of interest to scientists for many years. Condensed matter physicists, materials scientists, molecular physicists, and chemists have long been fascinated by the properties of matter under extreme conditions, and much effort has gone into determining their equations of state (EOS). Geologists, geochemists, and geophysicists have been drawn to this technique to study minerals at conditions found deep in the earth to help in understanding the dynamics of the core and mantle. Likewise, astrophysicists are concerned about the equations of state of planetary matter. Achieving extreme pressure and temperature conditions almost always requires furnaces, pressure cells, or diamond anvil cells (DACs) 32 and, in some circumstances, a combination of these. No matter which device is used to create the extreme conditions, the probe beam is required to traverse a considerable amount of material upon entering and exiting the sample. Hard x-rays provide excellent penetration power for such studies. In many cases, the available entrance and exit apertures are limited in size. The constricted exit aperture limits the angular range and, hence, the number of Bragg peaks over which data can be collected using monochromator radiation making structural identification ambiguous or impossible. It was recognized early that a white incident beam coupled with an energy dispersive detector set at a fixed exit angle can alleviate some of these difficulties. 33 This technique is limited in the ability to discriminate closely spaced Bragg peaks due to the energy resolution of the detector thus making complex structure determination difficult. Another approach to solving the structure of materials under extreme conditions would be the use of a high energy ( $>50 \mathrm{keV}$ ) monochromatic beam. This would compress the low order Bragg peaks into the forward direction and allow a (limited range) angle scan to be used.

\subsubsection{Requirements of the Beamline}

At the most extreme conditions, pressure and temperature gradients will exist in the sample with only a small portion of the sample at the maximum temperature and/or pressure. In high pressure DACs, the amount of material at the highest pressures typically has a cross-section of only several microns. 34 This means that the volume of material doing the scattering may be only several thousand cubic microns. Regardless of the technique used (white or monochromatic), the probe beam should have a small cross-section so that as many photons as possible strike the sample. Focusing a high energy white beam with total external reflection mirrors is difficult due to the low angles of incidence and, hence, long mirrors are required. So, high brilliance beams are a must for this type of experimental effort. If high energy monochromatic $x$-ray beams are used, an undulator source would be most appropriate. An undulator with the first harmonic at or above $50 \mathrm{keV}$ would almost certainly require an in-vacuum magnet system because the required gap would be on the order of $5 \mathrm{~mm}$ (see Section 3.3.2 - Insertion Devices in Type B Sectors). Such a device could have detrimental effects on the lifetime of the stored 
beam, but, with quasi-continuous injection (i.e., top-off) operation, beam loses could be compensated for and the current maintained at a near constant value.

The requirements for an in-vacuum ID clearly makes this type of beamline a candidate for a so-called APS specialized beamline (see Fig. 2.5.11). On a much smaller scale, the National Science Foundation supports the National High Pressure Facility at CHESS. Even on this first generation source, the National High Pressure Facility has had an important impact on research in the field of high pressure studies. A high pressure/high temperature facility can also be found on the Tristan Accumulator Ring (AR). This instrument, named MAX80, was originally installed on a Photon Factory bending magnet beamline. It was, however, moved to the AR in 1986 to take advantage of the increased critical energy of the emitted radiation.

\subsubsection{Kilometer Beamline for X-ray Imaging}

\subsubsection{Introduction}

Several kilometer-long beamlines have been proposed for ESRF and SPring-8, which are third generation synchrotron facilities under construction for scientific purposes similar to those of APS. A large area of illumination and a very high degree of collimation of $x$-ray beams at a sample are obtained without any special optics. The combination of these properties with the high brilliance of the third generation sources provides unprecedented $x$-ray beam properties at the sample, and it opens up new opportunities that have not been available previously. In addition, these long beamlines also allow experimenters to use geometry for image magnification or demagnification with fewer (or even without) optical components. We propose to construct a kilometer-long beamline at the APS for various imaging applications with a variety of stations located at different distances. Most of the applications are aimed at using the three properties discussed above, that is, a high degree of collimation, large area of illumination, and large geometry magnification factor. The common goal of all the imaging applications is to obtain submicron spatial resolution and time resolution. If only a 250-m long beamline could be constructed, its detailed layout is discussed in Chapter III where a Long Beamline Laboratory is described.

\subsubsection{Description of Programs and their Scientific Capabilities}

The main scientific programs proposed for a kilometer beamline include experiment stations for: (I) submicron resolution x-ray imaging , (II) submicron resolution and large area $x$-ray topography, and (III) submicron resolution microprobes. These programs will be conducted using three experimental stations located at about 50, 250, and 1000 meters from an undulator source (Fig. 2.5.12) whose peak brilliance is tunable between $4-40 \mathrm{keV}$ using the 1st and 3rd harmonics. The scientific capabilities of each of the three programs are discussed separately below. 


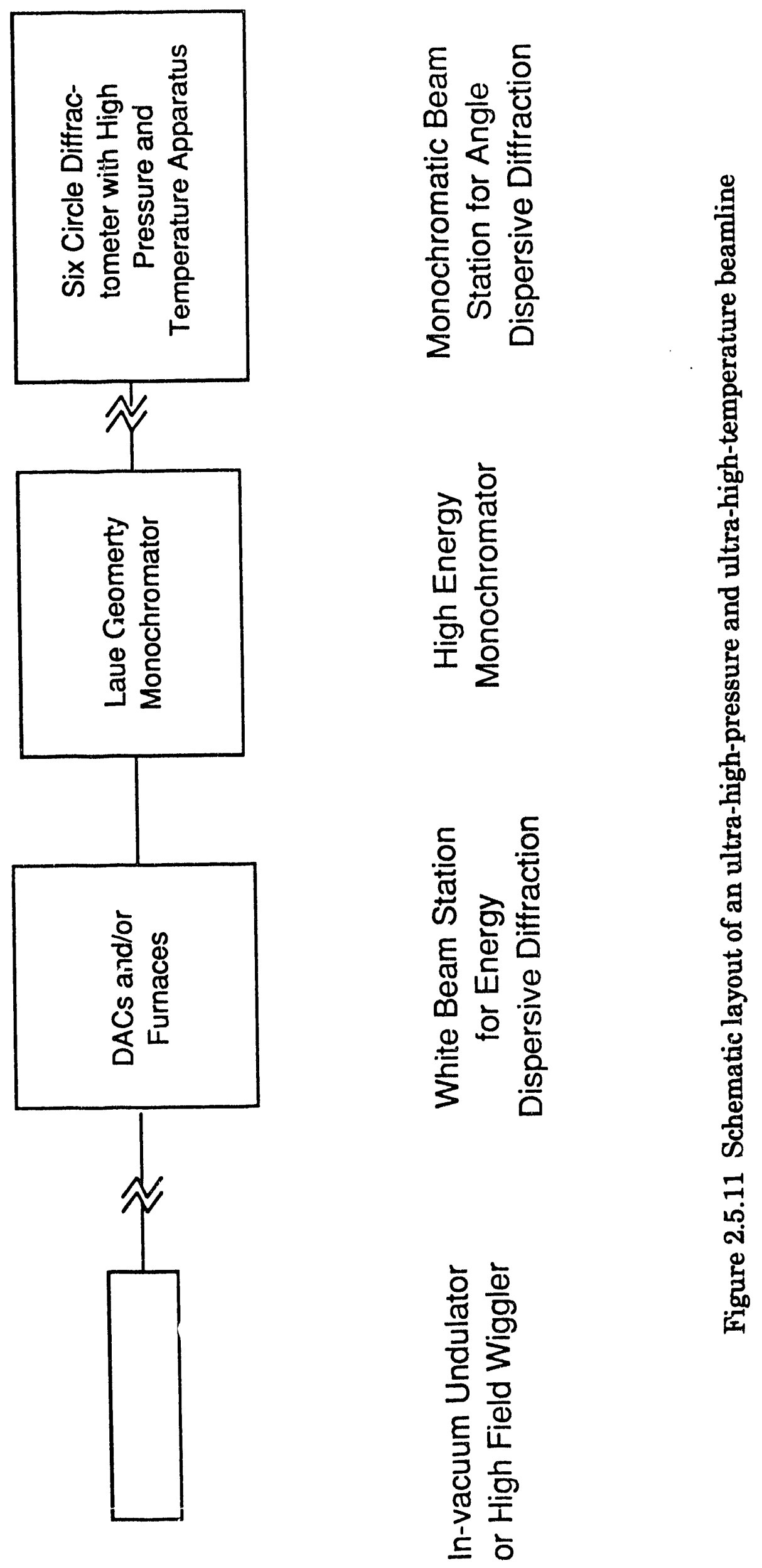




\section{(I) Submicron Imaging Using an X-ray Microscope}

The optical arrangement of the proposed x-ray microprobe (XM) is very similar to that developed by Dr. Schmahl's group (Gottingen, Germany) except that the $\mathrm{x}$ ray energies of interest here are about 2 orders of magnitude larger. A zone plate (ZP) of spatial resolution about 0.2 will be used to obtain a magnified image of the sample at the detector (Fig. 2.5.12). The magnification factor is roughly given by the ratio of the ZP-detector distance to the focal length of the ZP. For a ZP with the smallest zone width equal to $\mathbf{0 . 2}$ microns and the total number of zones equal to 1000 , the focal length of the ZP is about 1 meter for $8 \mathrm{keV} \mathrm{x}$-rays and increases to 5 meters for $40 \mathrm{keV} x$-rays. Therefore, the magnification factor of the image varies from about 40 for $40 \mathrm{keV} x$-rays to about 200 for $8 \mathrm{keV} \mathrm{x}$-rays. This high magnification is essential to obtain the spatial resolution of the ZP plate (i.e., 0.24 microns) because the image of a 0.2 -micron feature in the sample will be of a linear dimension of 8 microns for imaging with $40 \mathrm{keV} x$-rays. This image size is about a factor 2 of the photoelectron escape distance in Ge and only slightly larger than that in $\mathrm{Si}$. Time-resolved imaging is more easily obtainable because the imaging formation in an XM is parallel. Time resolution better than a millisecond should be possible at the APS.

\section{(II) Submicron Resolution and Large Area X-ray Topography}

A high degree of collimation of the illumination beam is very important in spatially resolved $x$-ray topography experiments. The divergence of the illumination $x$-ray beam at the high spatial resolution topography experiment station is better than $5 \times 10^{-7}$ in both the horizontal and vertical directions. Because the monochromator is the only optical component and it is located far away from the experiment station, the illumination beam is more stable than that obtained using optical means such as the use of multiple crystals. The large area of illumination also allows for the study of large crystals with high spatial resolution. These properties and the high brilliance of the APS undulator source open up many new possibilities not available previously. These new possibilities include: (1) the study of the dynamic nature of crystal defects formation, (2) the study of microstructures in multiphase materials and small precipitates, and (3) the structure and nature of dislocations on grain boundaries and interfaces.

\section{(III) Submicron Resolution Microprobes}

This experimental station will be used for various imaging techniques that do not require the large geometry factor necessary for (I) or the high degree of collimation and large area of illumination required for (II). The high brilliance of the undulator source is, however, required. Techniques to be developed include microdiffraction, microanalysis, microspectroscopy, and differential contrast microscopy. Submicron spatial resolution will be obtainable using Fresnel phase zone plates. This experiment station will also house the sample and focusing optics for the XM. 


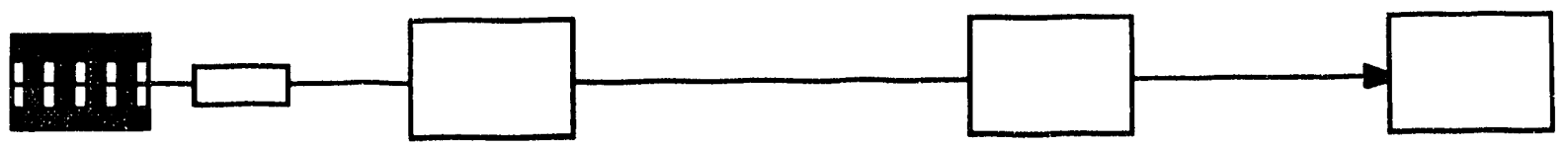

Monochromator

XM Detector Station

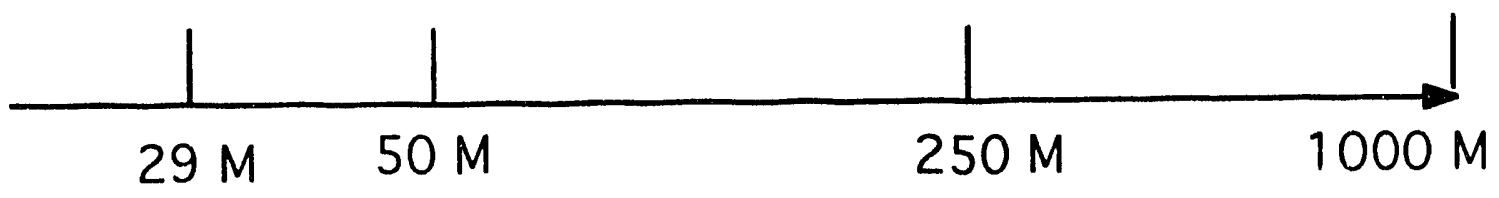

Figure 2.5.12 Requirements of a kilometer-long beamline for $\mathrm{x}$-ray imaging

\subsubsection{Chemical and Structural Kinetics Facility}

\subsubsection{Introduction}

Real-time imaging of physical phenomena is an exciting field for both pure and applied scientists. Unfortunately, because of flux-limited probes and lack of highspeed detectors, this field has not been fully exploited. The APS with its high brilliance has the necessary flux and small source size to perform these experiments. The small source enables one to uniformly control changes in samples under investigation. Further, with the development of high-speed detectors at the APS, we shall have the necessary tools to perform real-time imaging.

Structural and electronic changes in materials occur in the nanosecond to millisecond time range. 35 Most of the high speed, time-resolved studies performed in this time regime have been limited to pump-probe experiments in which the time resolution is defined by the time difference between the pump and the probe. $36 \mathrm{We}$ propose to set up a pump-probe station for time-resolved x-ray imaging. The instrumentation shall be designed for single-bunch $x$-ray scattering in which one can obtain two-dimensional data as a function of interbunch spacing (microsecond time scale) 37 and for multibunch, high flux imaging. Most basic and appliec' sciences like biology, 38 chemistry, materials science, 39 physics, and others, will benefit from it.

\subsubsection{Beamline Requirements}

This station shall be equipped with all tools necessary to perform a real-time imaging experiment, including a pulsed laser, high speed imaging detectors (now under development by us), high speed oscilloscopes, high pressure chambers, a 6 circle goniometer, and parallel processors with three-dimensional graphic 
capabilities (Fig. 2.5.13). Powerful computers are necessary not only for diagnostics during an experiment but also for data reconstruction. The demands on data storage capabilities will be high, considering that two-dimensional images from CCD detect.jrs can currently be collected and read out on a millisecond time scale. This corresponds to the generation of gigabytes of data every second! Faster and higher spatial resolution detectors are clearly on the horizon, and the data rate will only increase. Hence, the capability for large data storage is an absolute essential. Optics for this station will permit experiments to be performed with monochromatic $\mathrm{x}$-ray beams or with polychromatic beams for dispersive techniques, in which one c:an essentially collect data in a parallel mode (i.e., many wavelengths simultaneously). Dispersive optics are of a special class and will require a dedicated beamline to accommodate their configuration. We envision this facility to be the first user friendly system of its kind where one can come with only an idea and a sample.

\subsubsection{Biotechnology Development Facility}

\subsubsection{Introduction}

The proposed current phase beamlines for the APS include several that would be dedicated to solving the structures and elucidating the mechanisms of action of biological macromolecules. Research objectives range from fundamental studies of biological processes to the design of new pharmaceuticals. Techniques to be employed include crystallography (using monochromatic techniques, multiwavelength anomalous diffraction, and Laue diffraction methods), small angle scattering, and $\mathrm{x}$-ray absorption spectroscopy.

There is little doubt that demand for access to such beamlines will escalate over the next few years. The Human Genome Project, for example, will lead to a rapid increase in the number of sequenced proteins for which structures will be sought. Techniques such as site-directed mutagenesis can be used to produce variants of normal gene products, whose structures will also be of significant interest. New molecular biology techniques have made it possible to create samples of functionally specific DNA and RNA molecules in sufficient quantities for structural analysis. These considerations motivate early planning for the APS Instrumentation Initiative Biotechnology Development Facility.

\subsubsection{Beamline Requirements}

Much emphasis of the beamline design will be in generating focused beams with uniform illumination over small samples. Considerable effort will be dedicated to developing optics and detection systems that can measure the intensity distribution of the illuminating beam. Fortunately, such requirements are common to many of the special purpose beamlines discussed in this section.

Sample production, containment, and handling will be an important issue for this beamline. Considerable support facilities (which will become part of the beamline) will be needed for production and containment. Additional planning is needed for biosafety of samples handled at this facility. The sample handling will 


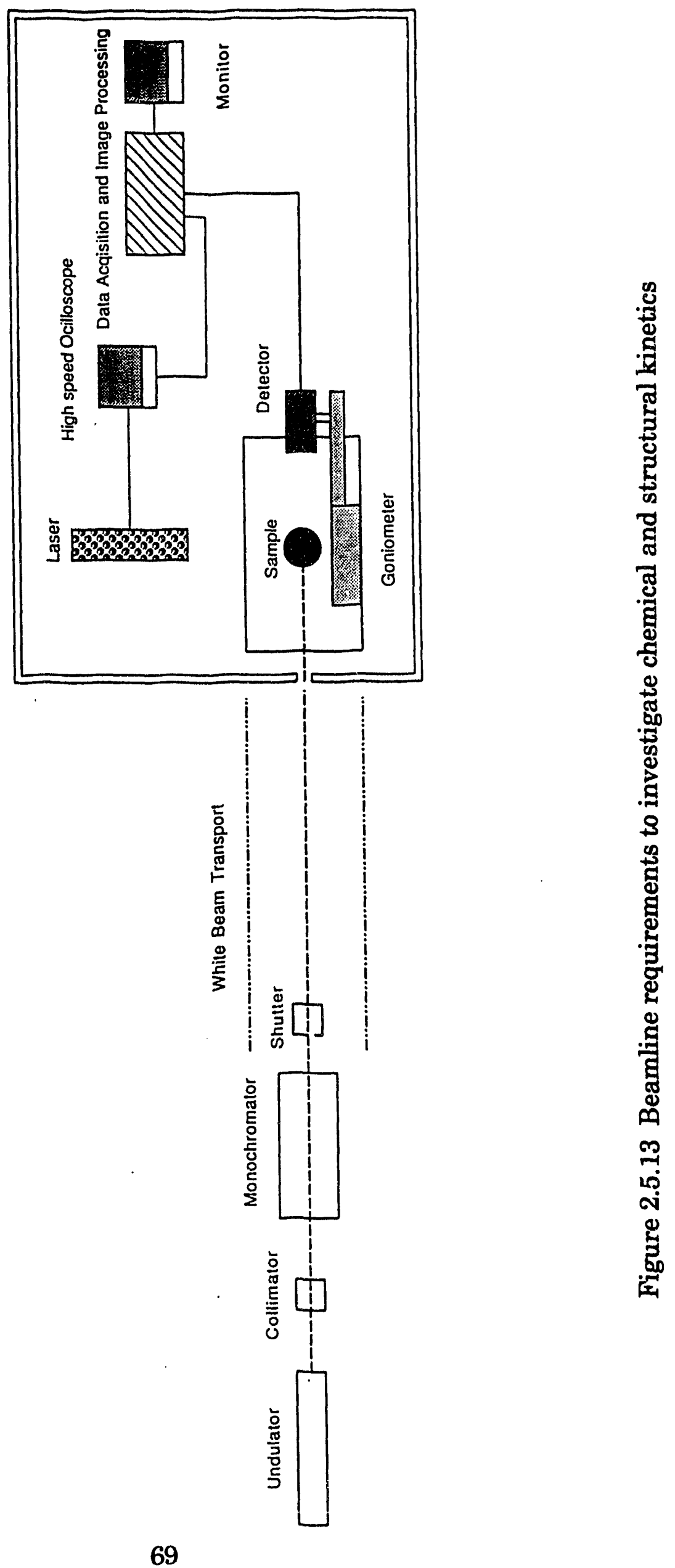


have to be streamlined using robotics, both for safety and efficiency of beam time utilization.

Commensurate with the requirements of this beamline is the development of sophisticated detector systems. Considerable $R \& D$ will be needed in this area with emphasis on both the collection and handling of large quantities of data.

For a major facility of this kind, a user friendly data analysis capability should be available. The techniques for evaluating complex biostructures with time efficiency are yet to be developed. These would make use of non-linear algorithms with necessary intelligence for evaluation.

\subsubsection{Development of X-ray Fabrication Process}

\subsubsection{Introduction}

$\mathrm{X}$-ray lithography has promised to be the fabrication technique for future generations of microelectronic devices. Many conventional and compact storage rings with critical energy around $1 \mathrm{keV}$ are specially designed for this application. Lithography with hard x-rays offers unique opportunities in producing very highaspect-ratio structures. For instance, micromachining has created a lot of interest in micro-mechanical parts such as micromotors, gear chains, and pressure sensors, all with dimensions much less than $1 \mathrm{~mm}$. Another example is hard x-ray zone plates, which will be essential for microfocusing and holography at the APS. They consist of high-aspect-ratio features that are not easily patterned with softer x-rays.

Another fabrication technique of increasing interest includes $\mathrm{x}$-ray-assisted processes such as chemical vapor deposition (CVD) or etching. In these cases, chemical reactions are caused by photoexcitation of the reactant gases and, possibly, the substrate. These processes have been shown to be low temperature techniques, which is essential for making devices of smaller size. It also offers important advantages such as anisotropic reaction, area selectivity, low damage, and low contamination. Currently, the reaction rate is about $1 \mathrm{~nm} / \mathrm{sec}$ using existing synchrotron sources.

\subsubsection{Motivation}

Hard $\mathrm{x}$-rays are particularly suited for high resolution, high-aspect-ratio lithography. The shorter wavelength means less diffraction and, thus, smaller features can be printed onto thicker photoresists. The higher energy photons are also more penetrating and can produce a uniform dose throughout the photoresist thickness, thereby eliminating the over-exposure problem. There are two requirements on the source for hard x-ray lithography, and they are both met by the APS: 1) small emittance to reduce geometrical errors like run-out and blurring, 2) high intensity so that the photoresist can be exposed in a reasonably short time. An additional advantage of the APS is the wide spectral range, which offers the flexibility of working in both soft and hard x-rays. Another area of interest for the APS is $x$-ray-assisted chemical process. Thin film deposition or photoetching are both possible. In particular, hard $\mathrm{x}$-rays at the APS can excite the K-shell electrons 
of many important elements like $\mathrm{Al}, \mathrm{Si}, \mathrm{Cr}$, or even $\mathrm{Ge}$, which can dramatically increase the rate of reaction. With additional energy tuning, chemical process can be selectively controlled by exciting the core level of a specific element.

\subsubsection{Beamline Design}

The beamline concept for the facility is shown in Fig. 2.5.14. On the bending magnet beamline, exposure can be performed with the conventional method of an $x-$ ray mask. Energy tuning is needed so that the spectral dependence of either fabrication process can be studied, and the initial operation can be done in the soft $x$-ray region. An arrangement of the double-crystal type will allow flexible adjustment of the energy window. Either a multilayer or a mirror/filter combination can be used in the low energy resolution mode for exposure. In the high energy mode, a pair of crystals can be used for in situ characterization of the thin film. A mask-wafer assembly will scan across the x-ray beam inside an exposure chamber. The exposure chamber should be separated from the upstream optics with a window, because the exposure chamber will be filled with $\mathrm{He}$ gas for lithographic work and with other reactant gases for x-ray-assisted processes.

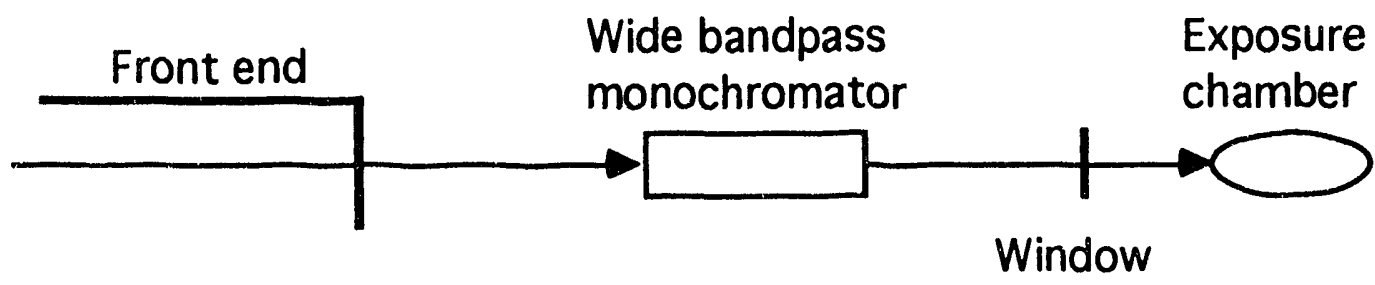

Figure 2.5.14 Principle of beamline for $\mathrm{x}$-ray microfabrication

For the ID beamline, a direct writing method may be used in place of the prewritten x-ray mask. The layout will be identical to the bending magnet beamline, with the addition of a zone plate after the monochromator to focus the x-rays to a spot of 0.1-0.2 $\mathrm{mm}$. This small spot will scan across the wafer to achieve direct writing capability. With an undulator optimized for energies lower than the standard Undulator A dose, the focus spot can attain photon flux that easily exposes regular photoresists like PMMA or can create the desired chemical reaction.

\subsubsection{X-Ray Manufacturing and Engineering Research Facility}

\subsubsection{Introduction}

Micromachining by synchrotron x-rays is usually understood to be a lithographytype of precision machining focusing primarily on microchip and microprocessor manufacturing and requires no introduction. A description of such a facility is presented in Section 5.3.11. The facility described in this section is a totally different, general purpose, ultra-precision engineering tool for any size machining and molding in diverse fields and applications. We believe that it has the capacity to revolutionize the machining and manufacturing industry and generate wide 
interest for participation as a CAT by the fast growing segment of industry involved in advanced laser-based manufacturing technique development. Both micro- and macro-engineering and manufacturing research will be possible on the proposed sector (lithography is excluded). The popular CNC milling is a case in point in terms of application. It will provide a significant advancement in photomachining processes (photosolidification using photosensitive polymers). A new process called "photonmachining" is proposed. It will incorporate advanced control machine systems. The Naw York University's well-known MOSAIC system at their Robotics and Manufacturing Research Laboratories, a Next Generation Workstation/ Machine Control (NGC) type system, may be used at the proposed facility in this sector.

Applications of micromachining engineering are wide spread in the areas of micromotors, microactuators, microsensors, and raicrotubing and valving systems. Currently, the end users are mostly in the medical, robotics, and sensor industries. Industry dollar volume is very large and rapidly increasing. At present, various lasers are the primary source utilized for 3D automated precision machining. Limiting factors associated with the use of lasers are beam shaping, total power, beam coherence and dispersion in media, non-penetrating 2D limitation (being a surface effect as opposed to volume effect with $\mathrm{x}$-rays), and a relatively short lifetime. Synchrotron x-rays offer superior beam shaping and control, precision, penetration, and power densities and thus will be a much more versatile tool for this purpose. Integrated 3D molding, machining, drilling, welding, selective surface coating and finishing are possible in one process in the manufacturing hutch. The stereo-lithography apparatus (engineering of 3D designs into 3D output) via an $\mathrm{x}$ ray source could revolutionize the making of prototypes, casting, pattern making, and tool and die industries in speed and complexity. Currently, stereolithography is used extensively in the computer, aerospace, automotive, and consumer product industries. Stereolithography apparatuses (SLA) produce prototypes through the process of photopolymerization, that is, transforming a liquid plastic polymer into a solid polymer by exposing it to an ultraviolet light laser source in an elaborate way. High thermal expansion of polymers limits the sizes and causes cracks and breakage-type failures. The x-ray SLA can dispense with these media limitations of the current SLAs. At present, there exist at least ten industry-scale SLA companies in the USA as well as those in Europe, Israel, and Japan, and the numbers are rapidly growing. Judging from this alone, we believe that there will be strong industry interest and participation in forming a CAT for a micro-macro-machining research facility such as that we are proposing here.

Reverse CAD research or coordinate measuring machines, (that is, generating dimensioned drawings directly from machined parts) is in large demand by the manufacturing industry and is another possible avenue of research with the proposed sector.

\subsubsection{Beamline Concept}

While the focused or unfocused white beam from IDs can apply any kind of power density with unparalleled size, precision, and stability in any engineering material, the bending magnet beams can be utilized as the stress relieving, 
sintering, heat treatment, and annealing sources. The proposed XEMR Sector schematic is shown in Fig. 2.5.15. Both the ID and BM lines will use white beams. The ID line FOE has a 5:1 P-B focusing mirror followed by a $1: 1$ focusing mirror. The first mirror diverts the beam by one degree to direct it to a second experimental station as explained below. The BM line $\mathrm{FOE}$ uses a 1:1 focusing mirror.

The ID beamline has three experimental stations. The farthest one (at $56 \mathrm{~m}$ ) uses a $1 \times 1$ focused beam to do $0.25 \times 0.35 \mathrm{~mm}$ size focused macromachining research. The hutch next to the FOE has two tables. The end table uses an unfocused beam at $0.35 \times 0.5 \mathrm{~mm}$ size for macromachining. The first table uses a diverted but focused beam (by one of the ID line mirrors) and is good for $30 \times 60 \mu \mathrm{m}$ size micromachining research. The $\mathrm{BM}$ line has two experimental stations. The farther one uses a focused beam of $0.3 \times 1.0 \mathrm{~mm}$ size for macro-processes. The one closer to the FOE uses an unfocused BM beam of $2.5 \times 200 \mathrm{~mm}$ size for $\mathrm{x}$-ray heat treatment, annealing, and stress relieving.

\section{Data handling and Communications}

\subsection{Introduction}

The Instrumentation Initiative experimental beamlines and anticipated research activities represent significant challenges in the areas of experimental data gathering, processing, storage, and transmission. New techniques, made possible by the high brightness of the APS and advances in time- and spatial-resolution of new sensors, will produce raw data in unprecedented volume. After the raw data is produced, three types of computer technologies are used to handle it: processing, storage, and transmission. Of these three, both processing power and transmission speed have been able to advance rapidly enough at reasonable cost to track the anticipated data production of the APS. The third technology, storage capacity, has not advanced as rapidly at reasonable cost. For this reason, techniques new to synchrotron radiation research will need to be developed and used to alleviate this bottleneck.

\subsection{Handling High Data Rates}

Very high raw data rates are common to high energy physics research, and, for many years, preprocessing and other techniques have been used to reduce the number of data sets saved and the amount of data retained. Preprocessing involves the use of relatively powerful computers to test the value of the data being produced. It can also be used to give a "quick look" at the final results by performing calculations similar to those planned for the final data reduction, but with less data or at reduced accuracy. In this way, the experimenter can adjust the apparatus to optimize results or decide to discard data previously saved due to its poor yuality. The processing power required to perform such quick look calculations, even at "super computer" levels, is becoming available at reasonable cost and will be common during the APS Instrumentation Initiative time period. 


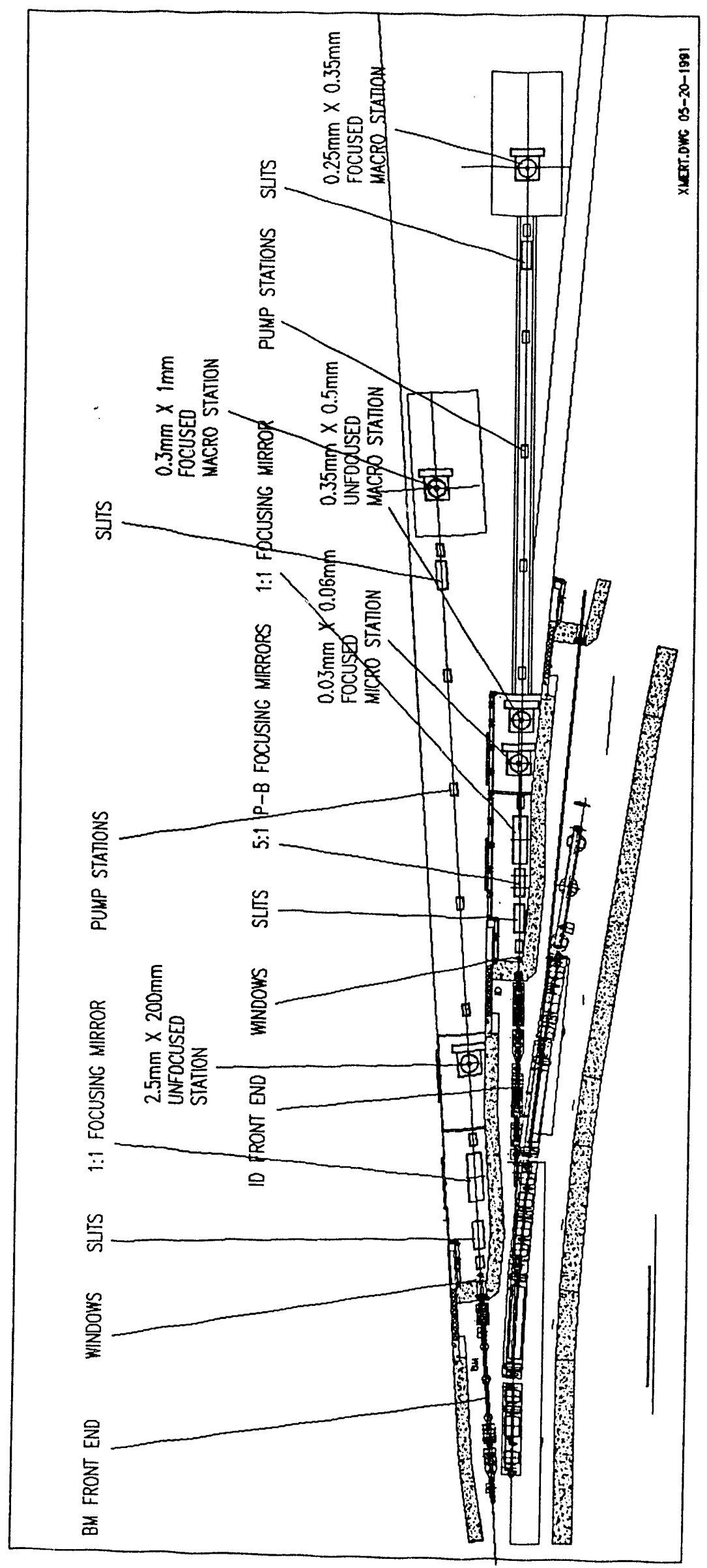

ปే 
Whether or not the average data quality can be enhanced by the above technique, other means may be necessary to address data volume. Large local storage will be needed to save data until off-peak periods of network usage in the same way that electric power users make sptimum use of that utility. This technique will insure that valuable data will not be lost due to temporary network congestion. Another technique that , $\mathrm{n}$ be used to reduce storage costs and network usage is data compression. Data compression algorithms can yield dramatic reductions in data volume, especially in two-dimensional fields of pixel data.

\subsection{APS Data Handling Infrastructure Improvements}

To help handle the anticipated data volume, two enhancements will be needed in the APS data handling infrastructure. First, the network system that serves the experimental area will be enhanced from the FDDI protocol (with a capacity of 10 M-bits/second) to Gigabit technology (with a capacity of about $600 \mathrm{M}$-bits/second). The second type of enhancement will be the provision of large capacity, hierarchical storage systems. These systems will perform an "impedance match" between the high volume of incoming data from high-speed, medium-volume, relatively expensive storage, such as RAM memory, to low-speed, high-volume, relatively inexpensive storage, such as optical disk media. Optical disk systems are ideal for archival storage because they are nonvolatile, time stable, and one tenth the cost of conventional disk memory. The media is inexpensive and compact, with about one gigabyte stored on an audio-type $\mathrm{CD}$, and is easily transported.

\subsection{Visualization Tools}

Another computer technology that will benefit APS experimenters in the mid90 's is that of visualization. These systems, based on high resolution, high performance graphic workstations, allow the user to view vast amounts of data presented in graphic form, with color and three-dimensional spatial format. During the current construction phase, beamline control methods will be developed to manipulate crystal orientations with high level, interactive tools. The use of sophisticated visualization tools will further enhance this interaction, and the superimposition of actual experimental data will enable a new level of real-time, experiment-to-results operation to be reached. These same visualization tools can be used to present the quick-look results (after some data reduction) to further verify proper operation.

\subsection{Control and Monitoring}

During the curre nt construction phase, the use of graphic workstations for all beamline apparatus control and monitoring will be fully developed. For the APS Instrumentation Initiative, this concept will be enhanced by the addition of the above uses of these same workstations for quick-look verification and experimental setup ana results visualization. The graphic workstation "X-windows" protocol allows the graphic results of data manipulation to be displayed on a computir and at a location completely different from where the data is being processed. The efficient transmission of screen data allows this separation to be small (elsewhere at the APS) or iarge (at the user's home institution or at a national super-computer 
center). The "X-windows" protocol will be exploited to present additional APS operations information and final results from data reduction processors both at Argonne and at the user's home institution. The concept can be further exploited to allow pre-testing of the experimental setup at the APS from the user's home institution.

\section{REFERENCES}

1 7-GeV Advanced Photon Source Conceptual Design Report, ANL-87-15 (1987).

2 G. K. Shenoy, P. J. Viccaro, and D. M. Mills, Characteristics of the 7-GeV Advanced Photon Source: A Guide for Users, ANL-88-9 (1988).

3 S. L. Xu, B. Lai, and P. J. Viccaro, APS Undulator and Wiggler Sources: MonteCarlo Simulation, ANL/APS/TB-1 (1992).

4 S. Yamamoto et al., Rev. Sci. Instrum., to be published.

5 P. Elleaume, Phys. Scripta T31, 67, 1990.

6 G. K. Shenoy, unpublished.

7 H. Kitamura and S. Yamamoto, Rev. Sci. Instrum., to be published.

8 G. K. Shenoy, P. J. Viccaro, and E. E. Alp, Rev. Sci. Instrum. 60, 1820 (1989).

9 A. K. Freund, M. Hart, J. R. Schneider, Nucl. Instrum. and Meth. A266, 247 (1988).

10 M. Blume, Appl. Phys. 57, 3615 (1985).

11 M. Blume and D. Gibbs, Phys. Rev. B37, 1779 (1988).

12 T. Ishikawa, Rev. Sci. Instrum. 60, 2058 (1989).

13 C. Malgrange, C. Carvalho, L. Braicovich, and J. Goulon, Nucl. Instrum. and Meth. A308, 390 (1991).

14 T. Ishikawa, K. Hirano, and S. Kikuta, J. Appl. Cryst. 24, 982 (1991).

15 W. Lowe et al., Phys. Rev. Lett. 67, 2513 (1991).

16 L. Carr, NSLS Newsletter, March, 1992.

17 M. H. Sher et al., Proceedings of the 8th International Conference on Ultrafast Phenomena, Antibes, France, $\mathbf{\delta}-1 \hat{2}$ June $\mathbf{1 9 9 \hat { z }}$. 
18 S. C. Davey et al., Proceedings of the OSA Short-Wave Coherent Radiation: Generation and Application Topical Meeting, Monterey, California, 8-10 April 1991.

19 J. D. Kmetec et al., Phys. Rev. Lett. 68, 1527 (1992).

20 Yu. I. Ostrovsky, M. M. Butusov, G. V. Ostrovskaya, Interferometry by Holography, (Springer-Verlag, 1980); D. Casasent, Optical Data Processing, (Springer-Verlag, 1978).

21 M. Sutton, S. G. Mochrie, T. Greytak, S. E. Nagler, L. E. Berman, G. A. Held, and G. B. Stephenson, Nature 352, 608 (1991).

22 G. Schmahl et al., X-ray Microscopy II, eds. D. Sayre, M. Howells, J. Kirz, and H. Rarback (Springer-Verlag, 1988).

23 M. Howells et al., Science 238, 514 (1987).

24 J. Kirz, H. Ade, C. Jacobsen, C.-H. Ko, S. Lindas, I. McNulty, D. Sayre, S. Williams, Rev. Sci. Instrum. 63, 557 (1992).

25 W. B. Yun, J. Chrzas, and P. J. Viccaro, Finite Thickness Effect of a Zone Plate on Focusing Hard X-rays, presented at the SPIE Conference (1990).

26 G. K. Shneoy and D. E. Moncton, Nucl. Instrum. Meth. in Phys. Res. A266, 38 (1988); G. K. Shenoy, P. J. Viccaro, and D. M. Mills, Argonne National Laboratory Report, ANL-88-9 (1988).

27 D. Attwood et al., Science 228, 1265 (1985).

28 N. M. Ceglio, J. X-ray Sci. and Tech. 1,7 (1989).

29 R. J. Field and R. M. Noyes, J. Chem. Phys. 60, 1877 (1974).

30 U. Bonse and M. Hart, Z. Physik 189, 151 (1966).

31 D. P. Siddons, C. Riekel, and J. B. Hastings, J. Appl. Cryst. 23, 401 (1990).

32 A. Jayaraman, Rev. Mod. Phys. 55, 65 (1983).

33 B. Buras, J. S. Olsen, L. Gerward, G. Will, and E. Hinze, J. Appl. Cryst. 10, 431 (1977).

34 A. L. Ruoff, Y. K. Vohra, W. A. Bassett, B. W. Batterman, and D. H. Bilderback, Nucl. Instrum. and Meth. A266, 344 (1989). 
35 For a review, see Handbook on Synchrotron Radiation, Vol. 3, edited by G. Brown and D. E. Moncton (Elseiver Science Publishers, 1991) chapter 9.

36 B. C. Larson, C. W. White, T. S. Noggle, and D. M. Mills, Phys. Rev. Lett. 48, 337 (1982).

37 D. M. Mills, Rev. Sci. Instrum. 60, 2338 (1989).

38 S. M. Gruner, Science 238 (1987).

39 R. Clarke, W. Dos Passos, W. Lowe, B. G. Rodricks, C. Brizard, Phys. Rev. Lett. 66, 21 (1991). 


\section{CHAPTER III USER FACILITY BUILDINGS}

\section{INTRODUCTION}

This section describes the buildings that support the various user facilities. All of these buildings are located outside the Experiment Hall. The structures are basically defined by the dimensional and functional requirements of the experimental programs of the users.

Four user laboratory/office modules to be constructed as part of the Instrumentation Initiative are identical to those being constructed in the current phase of the project. They are situated at predetermined locations and are spaced around the outer perimeter of the Experimental Hall to minimize the distance that experimenters must travel between their labs and offices and their beamlines.

It is anticipated that research programs based on $x$-ray imaging techniques will be conducted at the APS. These programs will support a separate building adequately designed to meet the requirements of this research.

During the planning of the APS construction project, the above requirements of the Instrumentation Initiative were anticipated. Hence, all the geotechnical evaluations were conducted as part the on-going construction.

Similarly, all the environmental compliance requirements for the entire site, which includes the Instrumentation Initiative, have been addressed prior to the beginning of the construction. The plans for the new initiative comply with all provisions of the National Environmental Policy Act and other related DOE orders and guidelines protecting the environment. Based on the APS Environmental Assessment (DOE/EA-0389) a Finding of No Significant Impact (FONSI) notice was established in the Federal Register, Vol. 55, No. 95 (5/16/90). The U.S. Army Corps of Engineers (COE) issued a permit for construction in wetlands in accordance with Section 404 of the Clean Water Act. As part of this permit, DOE consulted with $\mathrm{COE}$ on the implementation of a plan to mitigate a wetland loss of 1.8 acres total. A Flood Plains and Wetland Involvement Notice was published in the Federal Register (54FR 18326) on April 28, 1989. All construction, commissioning, and startup activities of the Instrumentation Initiative will be carried out in compliance with environmental permits, agreements, and DOE orders that provide the basis for the FONSI.

\section{LABORATORY-OFFICE MODULES}

The Lab/Office Modules (designated as Work Breakdown Structure Number 4.7.1) are one-story buildings located on the outer perimeter of Experiment Hall, and are intended to provide laboratory and office space for APS beamline user groups. The interior details of the Lab/Office Modules (or LOMs) provide flexible layouts responsive to each of the user groups'specific needs. 


\subsection{Architecture}

LOMs are single-story appendages with roof-level mechanical rooms located around the outer perimeter of the Experiment Hall. Each module, similar to that provided in the current phase of the construction project, will have a gross floor area of about 21,000 square feet. Each LOM is composed of eight laboratories that connect directly to the Experiment Hall, space for approximately 32 offices for the experimenters, meeting rooms, and support activities. Each module is served by a truck air lock.

Around the perimeter of the Experiment Hall, space has been provided for the construction of eight LOMs as shown in Fig. 3.2.1. Four LOMs are to be built in the current phase, and the remaining four are proposed to be built to support the Instrumentation Initiative. These four new LOMs are planned in anticipation of utilization of instrumentation proposed for the user beamlines operating in the new initiative. The LOMs being built in current phase are buildings $431,432,434$, and 435 . In the Instrumentation Initiative, buildings $433,436,437$, and 438 will be completed.

The interior of each module is organized by a longitudinal corridor that provides access to laboratories, offices, common facilities, and controlled entry points. Entry to the module is from the adjacent parking areas, which are already developed as part of the current construction. The LOMs can also be entered from the interior of the Experiment Hall. Emergency exits are provided at several locations around the building.

\subsection{Operational and Functional Needs}

The LOMs provide offices, laboratories (chemical and electronic), and common facilities for researchers working with the beamlines in the immediate vicinity. The common facilities located in the middle of the module provide shop space, toilet/locker rooms, a kitchen and break area, a meeting room, two rooms for APS and ESH staff, a secure room for electronic communication bridges, and a controlled access entry point. These facilities are conveniently located with respect to offices, laboratories, and the Experiment Hall. The office spaces will be located along the exterior walls, and the laboratories will be adjacent to the Experiment Hall in each module. At one end of each LOM, a floor-level truck air lock will be located. Additional space for the storage of liquid nitrogen and bottled gases is provided adjacent to the truck air lock.

\subsection{Structural Systems}

Design loads that have been planned for the structural design of the LOMs include dead loads, snow loads, live loads, wind loads, and seismic loads.

The structural system will consist of a steel beam, girder, and columns system supporting a steel-deck roof. Foundations will be conventional spread footings at building column locations with drilled cast-in-place concrete caissons and/or 


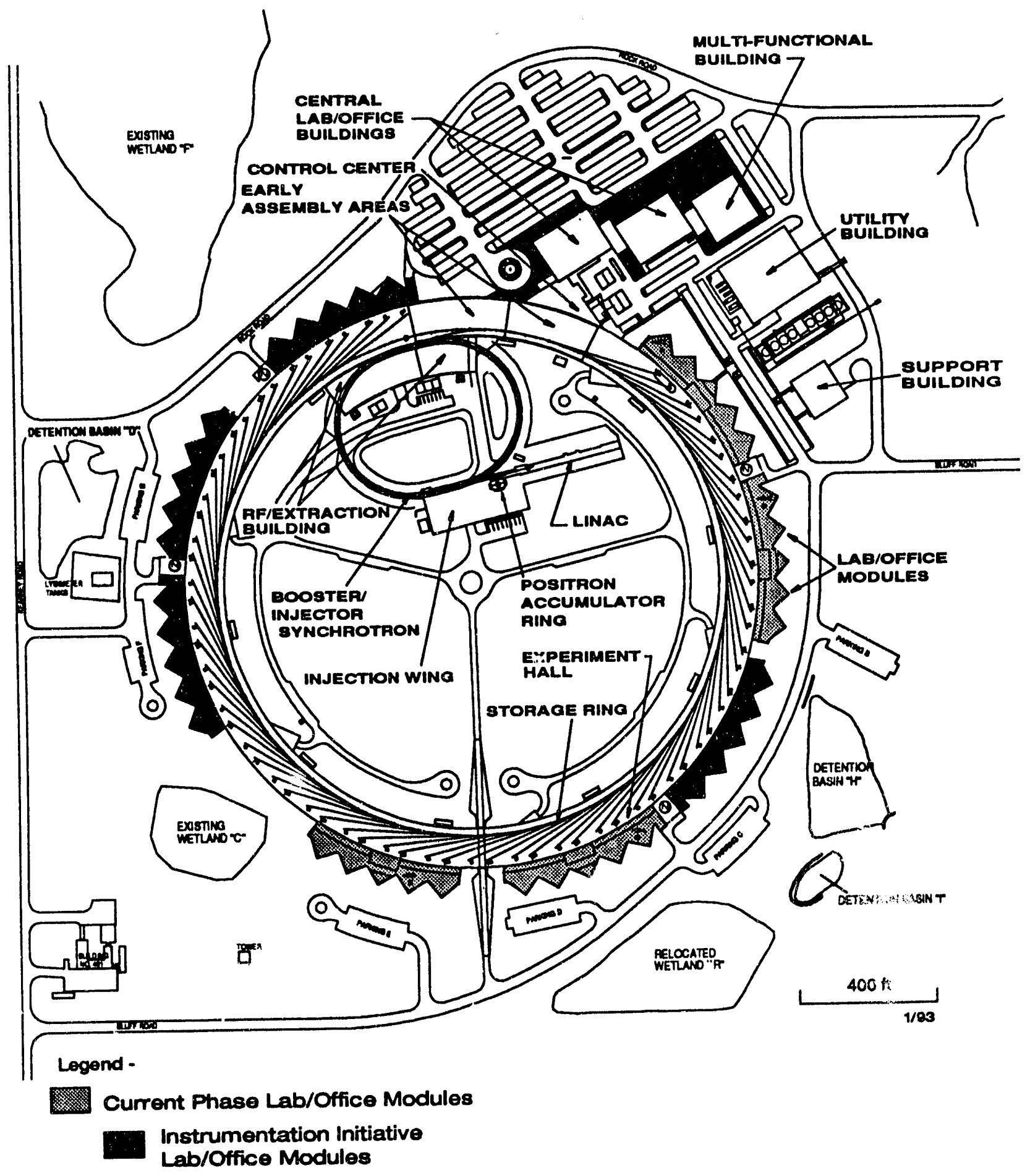

Fig. 3.2.1 Layout of the APS facilities showing the location of the Laboratory-Office Modules in the current construction phase and the Instrumentation Initiative 
continuous footings beneath the exterior walls. The floor will be reinforced concrete slab.on-grade over compacted granular fill.

The subsurface geology at the locations of the LOMs proposed in the new initiative has already been evaluated during the APS site subsurface exploration and geotechnical engineering.

\subsection{Heating, Ventilating, and Air Conditioning}

The office and laboratory areas of each module will be maintained at a space temperature of $75^{\circ} \mathrm{F} \pm 2^{\circ} \mathrm{F}$ year-round by a single variable-volume air-conditioning unit with return and exhaust fans located in the mechanical equipment room of each module. Each air-handling unit will consist of a low-pressure hot water, chilled water coil, filter, and economizer cycle.

Hot water will be delivered to the mechanical equipment room from existing headers in the Experiment Hall. Chilled water will be supplied from a connection to the utility loop in the Experiment Hall. A direct digital controller (DDC) in each module will control the HVAC equipment.

\subsection{Plumbing, Process Piping, and Fire Protection}

Plumbing systems planned for the LOMs consist of plumbing fixtures, electric water heating equipment, domestic cold and hot water distribution, sanitary and laboratory waste water collection, and vent piping systems in accordance with ANL requirements.

Process piping systems for laboratory cold and domestic cold and hot water, and compressed air will be provided.

A pad and piping will be provided for a vertical liquid nitrogen tank alongside each LOM.

Domestic cold water enters the building from the outside. Laboratory water and compressed air will be provided from the Experiment Hall.

A smoke control system utilizing the supply and return air fans will be provided. A wet-pipe automatic sprinkler system will be installed throughout. Hand-operated 15-pound carbon dioxide and 2.5-gallon pressurized water fire extinguishers will be located in accordance with NFPA requirements.

\section{E Electrical Power}

Electric power is supplied to each of the LOMs from an adjacent substation serving the Experiment Hall. The building distribution is $480 / 277$ volt, three phase, four-wire. Dry type transformers provide 480 volt to $208 / 120$ volt power. Lighting in the laboratories and offices consists of recessed fluorescent fixtures. Lighting in the shops, electric room, and other common areas will consist of fluorescent fixtures, pendant-mounted. 
A central energy management and control system (EMCS) will be provided. Emergency power is provided from standby diesel generator sets. Direct digital controllers (DDCs), a video terminal, and several events printers will be located in selected APS areas and will be connected to the existing energy management control system (EMCS).

All areas will be monitored by a conventional heat and smoke detection system connected to many zoned fire alarm control panels located in the APS buildings. The systems will be enunciated in the ANL fire station and the APS control center via dedicated telephone lines.

Telephone, paging, and electronic communication systems are provided. The electronic communication system will be run in conduit and cable trays shared with the telephone system. A central surveillance system will also be provided.

\section{X-RAY IMAGING LABORATORY}

\subsection{Introduction}

During the process of evaluating the user access proposals in 1992, the support facilities required by the user groups were better defined. The LOMs, which provide two standard laboratories and a set of offices, serve the principle requirements of most of the user groups performing fundamental investigations of unique problems in condensed matter physics, material science, chemical science, biological science, and engineering. On the other hand, the imaging technology groups, a new focal point at the APS, require additional facilities specifically designed to support the research activities in a fashion more akin to the R\&D environment of multi-disciplinary sciences. Also, this unique facility is based on a long beamline that extends beyond the walls of the Experiment Hall (see Fig. 3.3.1). With this in mind, a new x-ray imaging laboratory is proposed for construction as a part of this Instrumentation Initiative.

The X-ray Im g Laboratory (XIL, designated as Work Breakdown Structure Number 4.7.2) will consist of two buildings, one for housing the optics and research samples, and the other for housing the detector and data collection instrumentation (see Fig. 3.3.2). This feasihility design will be limited to defining the requirements on space and type of buildings. The final experimental plan will finalize the interior details of t'nese buildings.

\subsection{Architecture}

The XIL consists of two self-standing buildings connected by a beamline that extends from the wall of the Experiment Hall. The XIL will have a gross floor area of about 10.000 square feet, of which about 6000 square feet will be for the optics building and 4000 square feet will be for the detector building. The optics building will contain space for offices, laboratories, and mechanical/electrical 


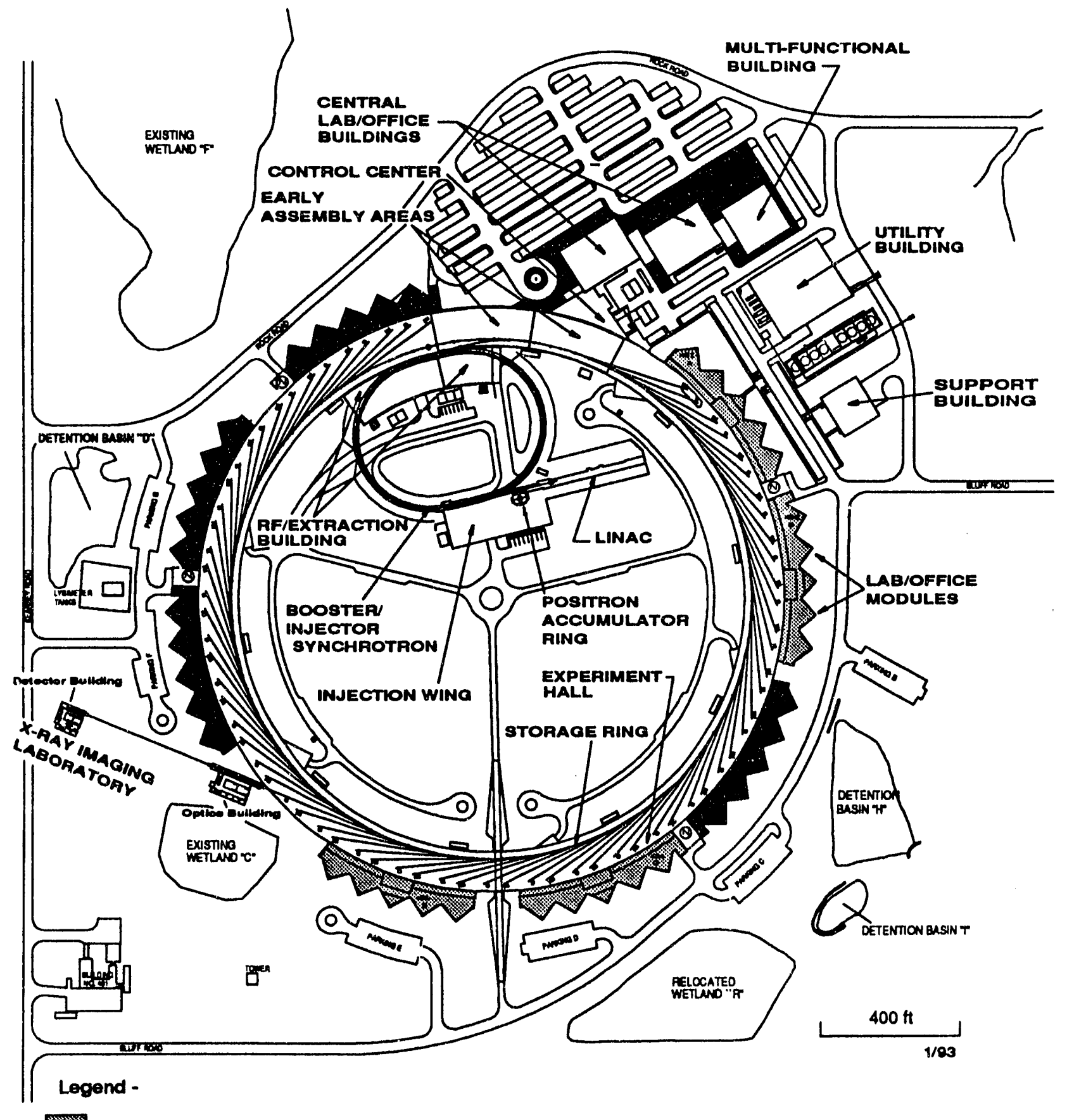

Current Phase Lab/Office Modules

Instrumentation Initiativo Lab/Office Modules

Fig. 3.3.1 Location of the X-ray Imaging Laboratory on an ID source. The beamline extends from the perimeter of the Experiment Hall in a north-westerly direction 


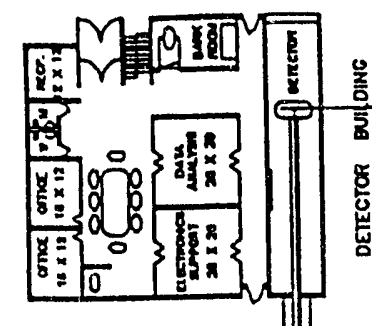



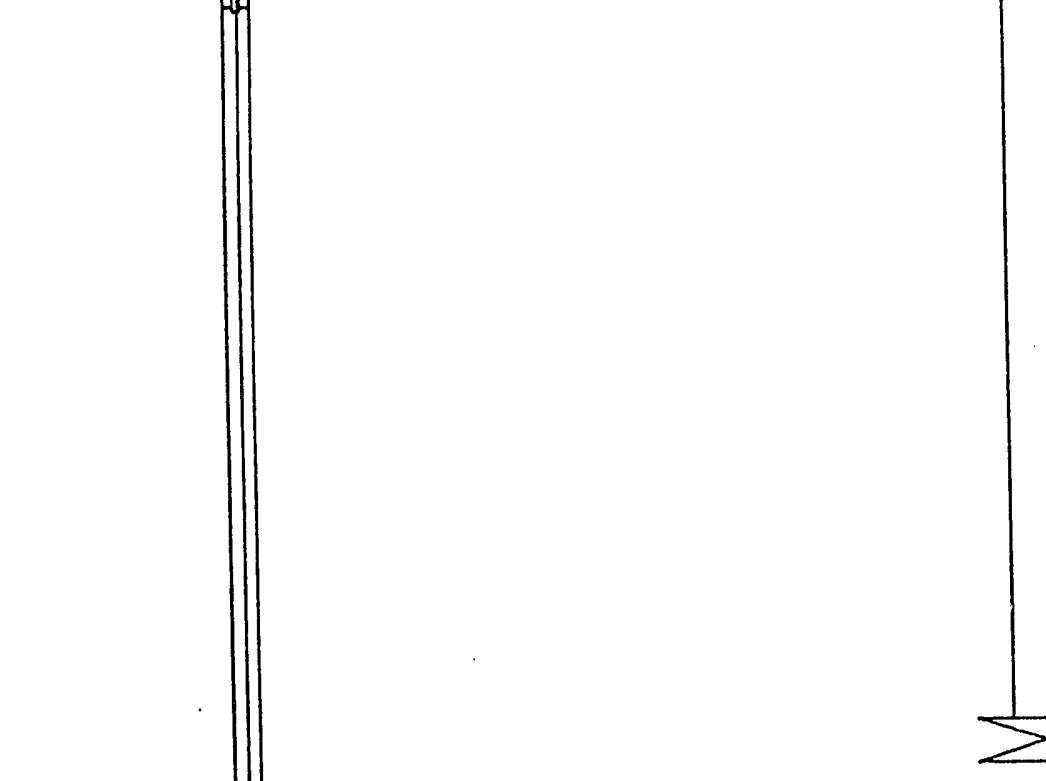

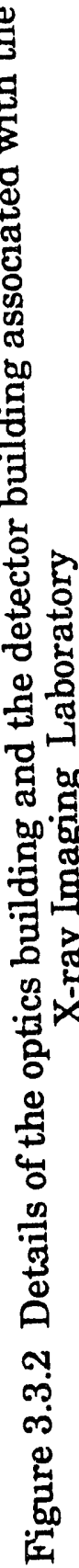

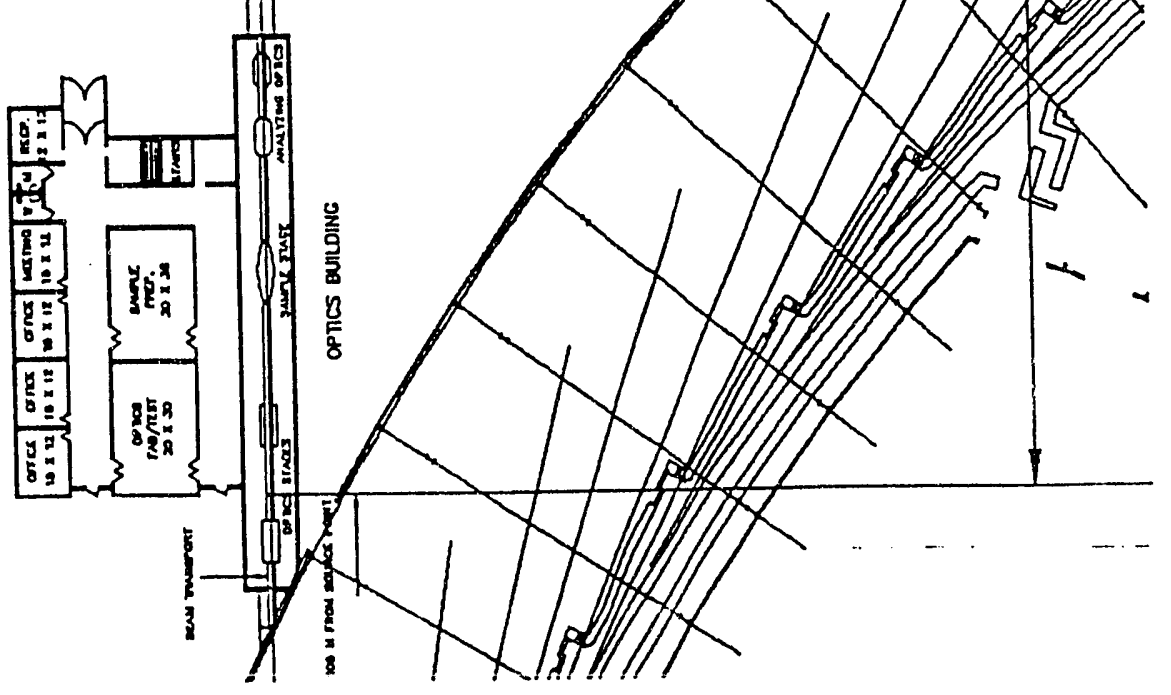


rooms. The detector building will contain two laboratories and four offices, general support space, and mechanical/electrical rooms.

The beamline will be located at experimental Sector number 21. This beamline will use the radiation from the ID in Sector 21 and will extend from the perimeter wall of the Experiment Hall near column number 130 to the optics building.

\subsection{Operational and Functional Needs}

The optics laboratory will receive the radiation using a beam transport line. The beam transport will be appropriately mounted and shielded. The ends of the beam transport are solidly fastened to the building structure at either end to prevent its movement. In addition, the transport will be evacuated to $10^{-6}$ Torr with pumping systems strategically located along the beamline. The radiation will be handled in the main optics station, which is an extended room with the capability to include different sets of optical elements and the sample for investigation. There will be space for support laboratories for the fabrication and testing of optics and for sample preparation. Additional space has been planned for offices and support needs. The detector laboratory will contain the detector station, electronics laboratory, a dark room for photographic development work, a data analysis laboratory, and four offices. The analyzed radiation beam will be transported from the optics laboratory to the detector laboratory with appropriate shielding. Again, this transport will be supported at various places along the length of the beam pipe and at the ends. Evacuation of the team transport to 10-6 Torr will be provided by vacuum pumping systems.

Both the optics and detector laboratories will have mechanical and electrical rooms on the upper level accessed through a stairwell.

\subsection{Structural Systems}

Design loads that have been planned for the structural design of both buildings in the XIL include dead loads, snow loads, live loads, wind loads, and seismic loads.

The structural system for both the optics and detector buildings will consist of a steel beam, girder, and columns system supporting a steel-deck roof. Foundations will be conventional spread footings at building column locations with continuous footings beneath the exterior walls. The ground floor will be reinforced concrete slab-on-grade over compacted granular fill; the roof level mechanical and electrical rooms will have a concrete floor supported by steel decking.

The subsurface geology at the locations of the entire XIL has been evaluated during the APS site assessment as a part of subsurface exploration and geotechnical engineering. 


\subsection{Heating, Ventilating, and Air Conditioning}

All the laboratory and office areas of each of the XIL buildings will be maintained at a space temperature of $75^{\circ} \mathrm{F}+2^{\circ} \mathrm{F}$ year-round with a variablevolume air-conditioning unit with return fans located in the mechanical equipment room of each building. Each air-handling unit will consist of a low pressure hot water coil, chilled water coil, filter, and economizer cycle.

Chilled water and hot water will be supplied from a connection to the Experiment Hall that extends through the adjoining LOM. A direct digital controller (DDC) in each module will control the HVAC equipment.

The upper level mechanical equipment room will be ventilated in summer and heated in winter to maintain a space temperature of $55^{\circ} \mathrm{F}$.

\subsection{Plumbing, Process Piping, and Fire Protection}

Plumbing systems planned for the XIL buildings consist of plumbing fixtures, electric water heating equipment, domestic cold and hot water distribution, sanitary and laboratory waste water collection, and vent piping systems in accordance with ANL requirements.

Process piping systems for laboratory cold, and domestic cold and hot water, laboratory waste, and vent compressed air will be provided.

Domestic cold water enters the building from an existing outside line. Laboratory water and compressed air will be provided from the LOM.

A smoke control system utilizing the supply and return air fans will be provided. A wet-pipe automatic sprinkler system will be installed throughout. Hand-operated 15-pound carbon dioxide and 2.5-gallon pressurized water fire extinguishers will be located in accordarice with NFPA requirements.

\subsection{Electrical Power}

Electric power is supplied to each of the XIL buildings from an adjacent substation serving the Experiment Hall. The building distribution is $480 / 277$ volt, three phase, four-wire. Dry type transformers provide 480 volt to $208 / 120$ volt power. Lighting in the laboratories consists of recessed fluorescent fixtures. Lighting in the common area will consist of fluorescent fixtures, pendantmounted.

A central energy management and control system (EMCS) will be provided. Emergency power is provided from standby diesel generator sets. Direct digital controllers (DDCs), a video terminal, and several events printers will be located in selected APS areas and are connected to the existing energy management control system (EMCS). 
All areas will be monitored by a conventional heat and smoke detection system connected to many zoned fire alarm control panels located in the APS buildings. The systems will be enunciated in the ANL fire station and the APS control center via dedicated telephone lines.

Telephone, paging, and electronic communication systems are provided. The electronic communication system will be run in conduit and cable trays shared with the telephone system. A central surveillance system will also be provided. 


\section{CHAPTER IV COST AND SCHEDULE}

\section{INTRODUCTION TO COST AND SCHEDULE}

All costs for the Instrumentation Initiative are developed from the Work Breakdown Structure (WBS) and are expressed in FY 1991 dollars. The WBS encompasses all elements of the construction phase from the design to the end of construction. The experimental facilities technical components were designed by the staff of the APS Experimental Facilities Division (XFD), who also estimated their costs. The Conventional Facility designs were developed by APS staff and the architect/engineering firm of Lester B. Knight, while the cost estimates were developed by the construction contractor, Rust Engineering, and the APS staff. The costs of Laboratory-Office Modules are based on actual bids obtained during the current phase of construction. A complete planned WBS is shown in Table 4.1.1. The WBS shows the Instrumentation Initiative at level 1. Level 2 shows the functional responsibility that includes the experimental and conventional facilities. Also included at this level are project management overheads and contingency. The technical components of the experimental facilities are defined at WBS level 3 (e.g., 4.4.1). All costs of materials, construction, and the engineering, design, and inspection (ED\&I) are estimated at an appropriate WBS level and rolled up to develop the final costs at WBS level 2.

The cost estimates include all costs for PACE construction to be incurred after the Instrumentation Initiative approval. The scope of this initiative includes construction of equipment behind the shield wall for nine additional sectors, support to construct five special purpose beamlines, four lab-office modules for the users, and one $x$-ray imaging laboratory.

It should be pointed out that twelve special purpose beamlines have been described as examples in Chapter II. In selecting the final five beamlines for the Instrumentation Initiative, these examples as well as other ideas from the user community will be used. Typical costs for each of these beamlines is about 4 to 8 million dollars. In the current package, only part of this cost is requested with the assumption that the rest will be funded from other avenues, such as federal agencies, non-federal agencies, and industry. This approach is similar to that used in the current phase where it was very successful.

The costs for R\&D in support of construction, operations, and for commissioning the experimental facilities are also included in this cost estimate.

The time estimated for the conventional facilities engineering, design, and construction is based on the experience gained in these activities during the ongoing phase of the Project. It is estimated that development of the Instrumentation Initiative technical components will start with the generic-type insertion devices and front ends at the same time as the technology for these devices is being developed in the current phase. The specialized insertion devices and front ends will be developed after the completion of initial phase of construction. 
Table 4.1.1

APS Instrumentation Initiative Work Breakdown Structure

WBS Code Element Description

X.4.0

APS Instrumentation Initiative

X.4.1

Project Management (Instrumentation Initiative)

X.4.4

Experimental Facilities

X.4.4.1

Experimental Facilities Technical Components

X.4.4.1.1

Insertion Devices

$\mathrm{X} .4 .4 .1 .1 .1$

Type A Insertion Devices (4)

$\mathrm{X} .4 .4 .1 .1 .2$

Type B insertion Devices (5)

X.4.4.1.2

Beamline Front Ends

$\mathrm{X} .4 .4 .1 .2 .1$

Type A Front Ends (4)

$\mathrm{X} .4 .4 .1 .2 .2$

Type B Front Ends (5)

X.4.4.1.5

Special Purpose Beamlines (5)

X.4.7

Conventional Facilities

$\mathrm{X.4.7.1}$

Lab Office Modules (4)

X.4.7.2

$\mathrm{X}$-ray Imaging Lab (1) 


\section{Cost Estimates}

The direct cost elements such as labor, materials and services, direct material, and subcontracts are broken down into various craft codes. These have been listed in Table 4.2.1. The rates used for the various crafts are given in Table 4.2.2 as hourly rates in FY 1991 dollars.

The work in each element of the WBS is divided into eight activities: design, procurement, fabrication, assembly, testing, installing, checkout, and Level of Effort (LOE). The last activity, namely $\mathrm{LOE}_{\text {, }}$ is the administrative effort involved in performing the work and does not have a true final product.

All the overhead and paid-absence costs for the cunstruction activity are included separately along with the rest of the APS construction project. However, in deriving the man-year numbers, the paid-absence fraction is included.

A cost breakdown by WBS code for the Instrumentation Initiative is shown in Table 4.2.3 in FY 1991 dollars. The cost estimates for the conventional facilities include two elements: 1) the cost of architect/engineering services, and 2) construction costs.

All cost estimates for the experimental facilities technical components include three basic elements: 1) costs of hardware deliverable (materials), 2) construction costs, and 3) ED\&I costs. These elements are given in detail in Section 4. 
Table 4.2.1

\section{COST ACCOUAT PIAHARG - DIRECT COST ILIMENTS}

\section{Labor Classifications - ies}

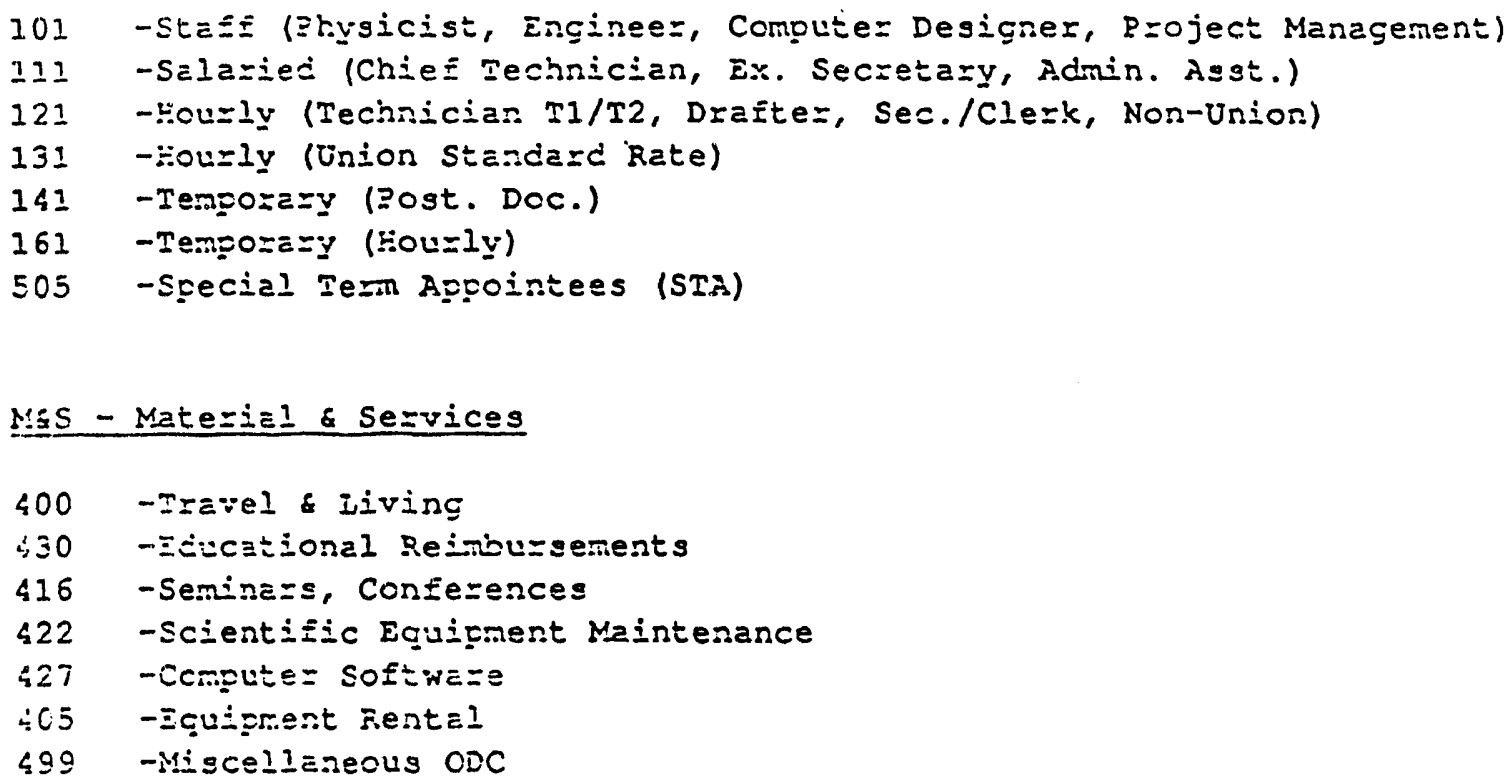

ANí Sevice Centers

\begin{tabular}{|c|c|}
\hline 521 & -FNI Cert:zal Shons \\
\hline $5 \pi i$ & --Hi Eiectzonics Divisica \\
\hline 561 & 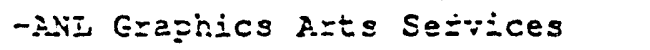 \\
\hline $5 \Xi 0$ & -ivi Corputer Sezvices Division \\
\hline 520 & - Eij Engineezirg Di̛isicn \\
\hline $5 \subseteq 0$ & -2ri - pll otrez suspo=t \\
\hline
\end{tabular}

\section{IEZECะ $\because E \pm E= \pm \equiv I$}

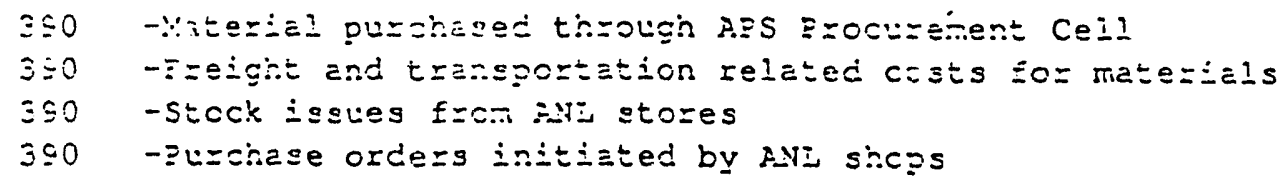

\section{$\operatorname{siccsic}=a \cos$}

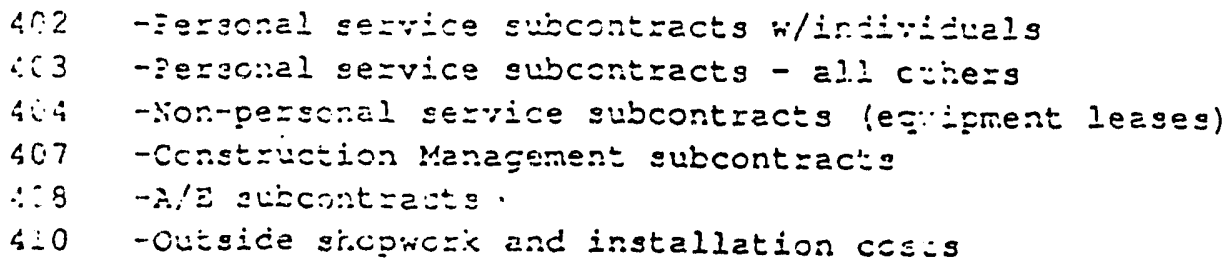


Table 4.2.2

Craft Codes and Hourly Rates (FY 92\$)

Engineering. Design and Inspection (ED\&I):

101-PH -Physicist

101-EN -Engineer Mechanical and Electrical

101-SW -Computer Designer (Software)

101-DC -Designer/Design Coordinator

121-DR -Drafter

141- -Postdoc

$\$ 33.40$

$\$ 33.40$

$\$ 33.40$

$\$ 33.40$

$\$ 17.46$

$\$ 16.72$

Construction:

Assembly and Test: ${ }^{* *}$

121-T1 -Technician, relatively unskilled

$\$ 17.46$

121-T2 -Technician, experienced/skilled

$\$ 17.46$

Fabrication/Manufacturing:

521- - ANL Fabrication/Manufacturing Support

$\$ 51.80$

Installation Subcontracts ("Davis-Bacon"): ${ }^{* *}$

410-IP -Plumber,Steam Fitter Sheet Metal

410-IE -Electrician

410-IC -Carpenter

410-IT -Technician

$\$ 45.00$

$\$ 45.00$

$\$ 45.00$

$\$ 45.00$

Level of Effort (LOE):

101-MN -Project Manager

$\$ 33.40$

121-SS -Secretarial Support

101-EN -Project Engineer

* Includes effective hourly base pay and benefits, but does not include lab overheads.

** Includes hourly base pay, benefits, and overheads, and profit for "outside" vendor or contractor. 


\section{APS INSTRUMENTATION INITIATIVE COST BREAKDOWN BY WBS CODE}

\section{WBS Code}

\section{Description}

APS Instrumentation Initiative

4.0

4.1

4.4

4.4.1

4.1.1.1

4.4.1.1.1

4.4.1.1.2

4.4.1.2

4.4.1.2.1

4.4.1.2.2

4.4.1.5

4.7

4.7.1

4.7 .2

Experimental Facilities

Insertion Devices

Type A Insertion Devices (4)

Type B Insertion Devices (5)

Beamline Front Ends

Type A Front Ends (4)

Type B Front Ends (5)

Special Purpose Beamlines

Conventional Facilities

User Lab-Office Modules (4)

$X$-ray Imaging Laboratory (1)
Project Management (2\% of Construction Cost)

Experimental Facilities Technical Components

Overheads $(5 \%)$

Contingency (18.5\% of Construction Cost)

Total Estimated Cost (TEC)

R\&D in Support of Construction

Other Project Costs

Inventories

Total Project Cost (TPC)

\section{Cost in FY $92 \mathrm{MS}$}




\section{TIME SCHEDULE}

The schedule is based on the assumption that the conventional facilities have to be completed in time for current phase operations to support users of the facility. Therefore, the schedule allows for some overlap between the two phases for continuity and efficiency. The experimental facilities technical components will be using existing facilities from the current phase. Therefore, there will be no conflict of schedule. The design, construction, and installation time schedule is based on experience of personnel at APS and other laboratories as well as vendor quotations. The installation of the technical components behind the shield wall is scheduled to coincide with the shutdown and maintenance schedule during operations.

Figure 4.3.1 shows the time schedule estimated for both the conventional and the experimental facilities.

\section{Cost ScheduLE}

Using the detailed WBS cost estimate in Table 4.2.3, which contains the cost of materials and labor of various crafts, and the time schedule shown in Figure 4.3.1, developed for completion of these activities, a cost schedule profile has been generated and is shown in Table 4.4.1 and Figure 4.4.1. In addition to the cost elements previously mentioned for both the conventional and experimental facilities, included in this profile are: 1) laboratory overheads estimated at $5 \%$ of construction costs for indirect expenses (such as procurement, accounting, human resources, etc.) and maintenance of facilities, 2) contingency costs estimated at $20 \%$ of construction cost, because the cost of the specialized insertion devices, front ends and the special purpose beamlines may vary.

Applying the escalation rates given in Table 4.4.2, the escalated cost schedule profile shown in Table 4.4.3 was generated and is graphically presented in Figure 4.4.2.

The budget BA/BO profile (Budget Authorization/Budget Obligated) for the construction project is broken down by fiscal year and is shown in Table 4.4.4 and Figure 4.4.3. 


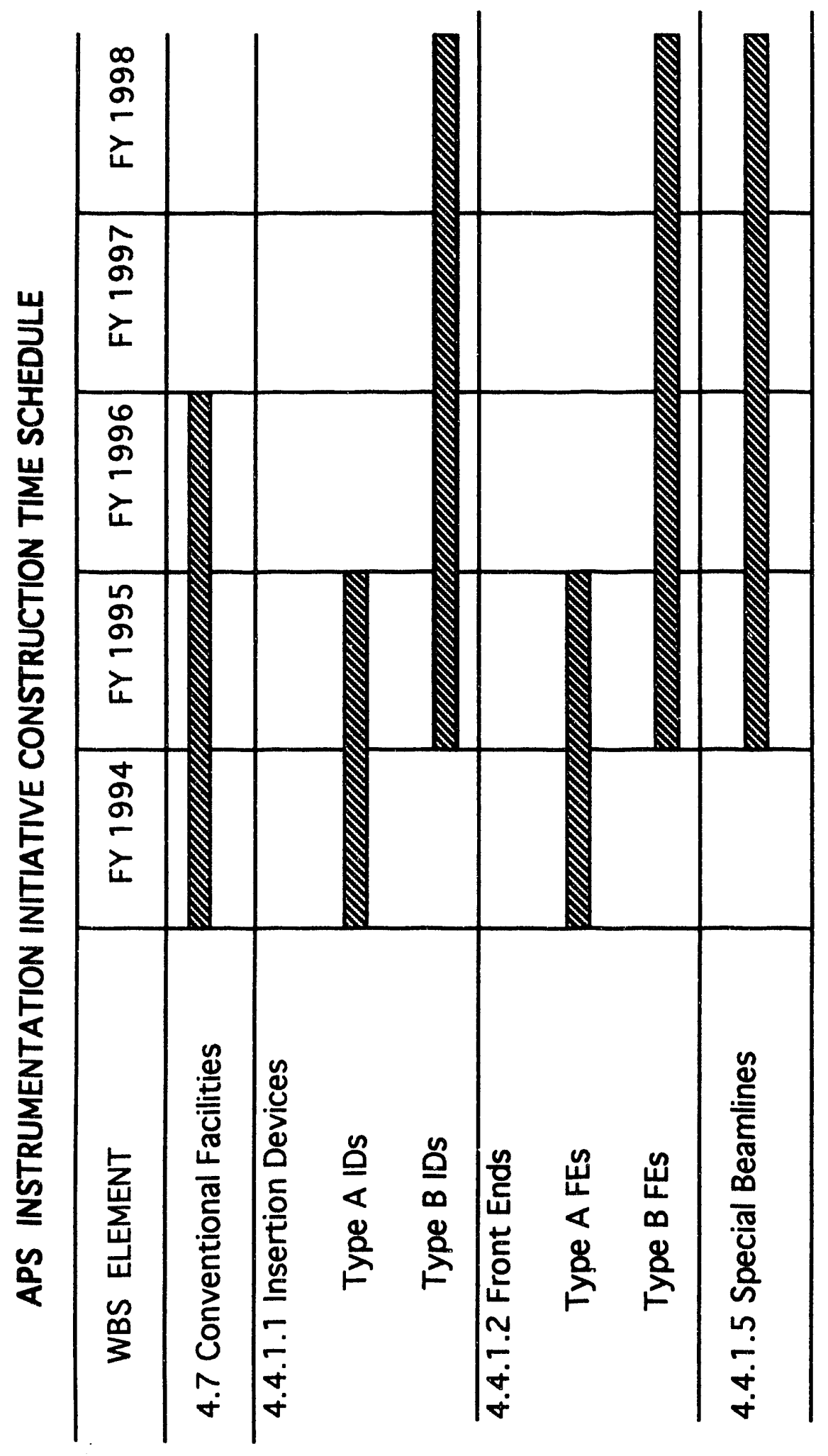

官 


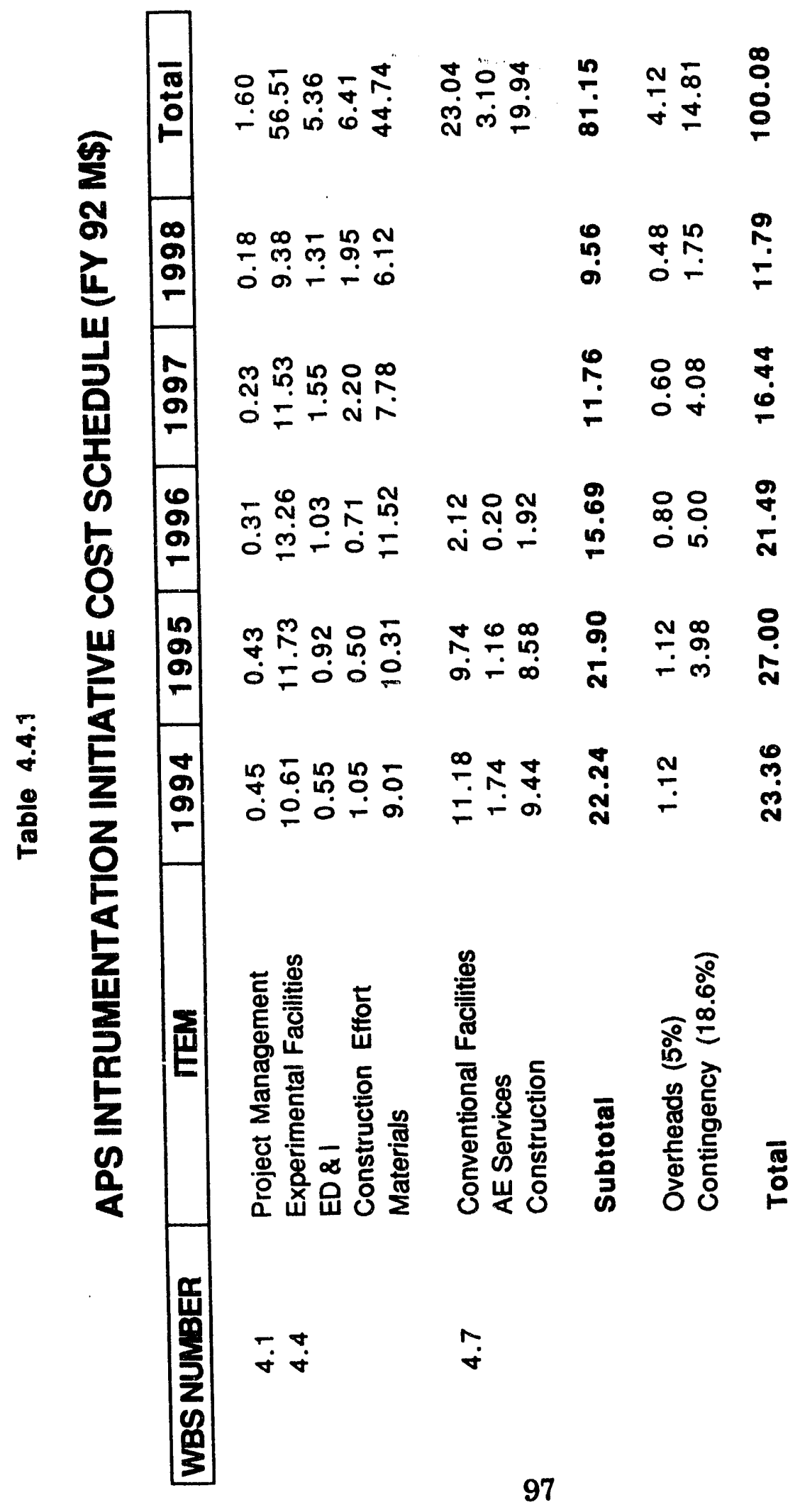



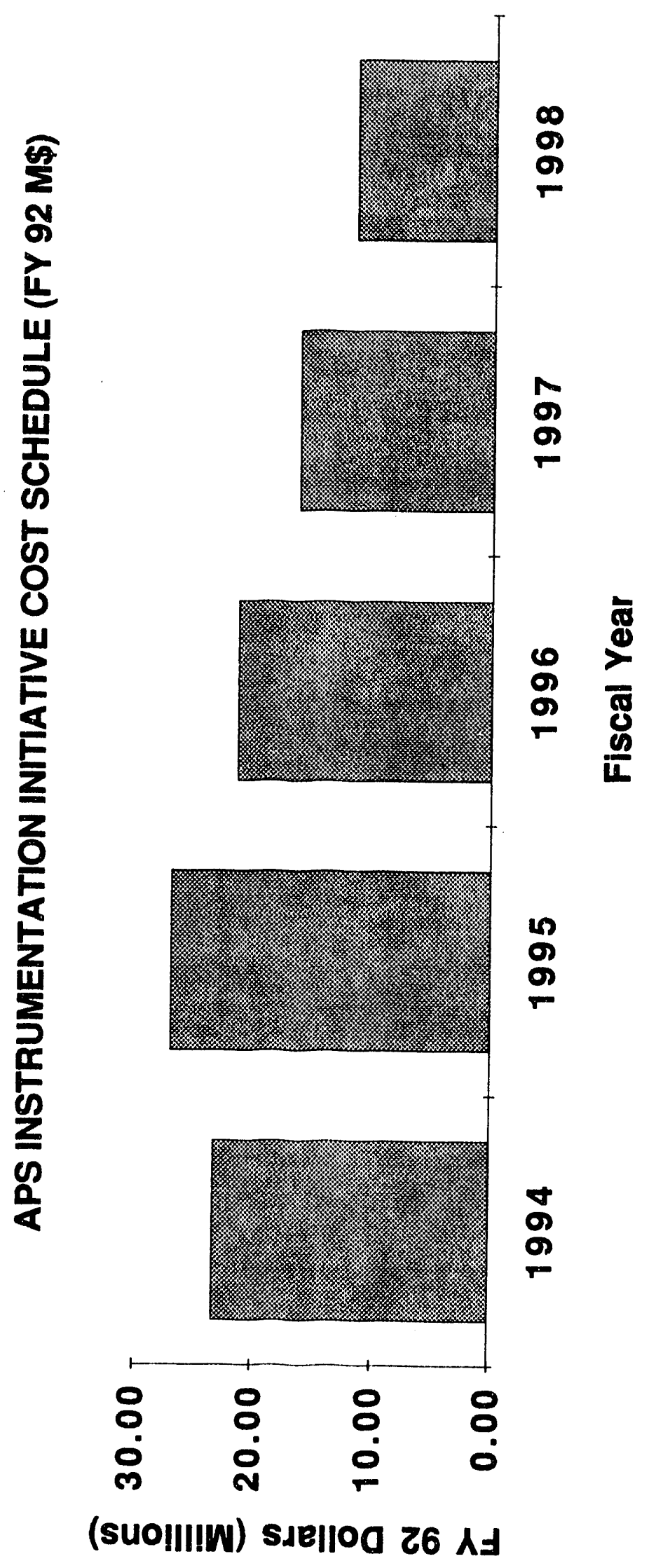
Table 4.4 .2

\section{Escalated Rates}

\begin{tabular}{ccc}
\hline Fiscal Year & $\begin{array}{c}\text { Inflation } \\
(\%)\end{array}$ & $\begin{array}{c}\text { Escalation } \\
\text { Factor }\end{array}$ \\
\hline & & \\
1992 & - & 1.000 \\
1994 & 7.0 & 1.070 \\
1995 & 4.1 & 1.114 \\
1996 & 4.4 & 1.163 \\
1997 & 4.5 & 1.215 \\
1998 & 4.4 & 1.269 \\
\hline
\end{tabular}




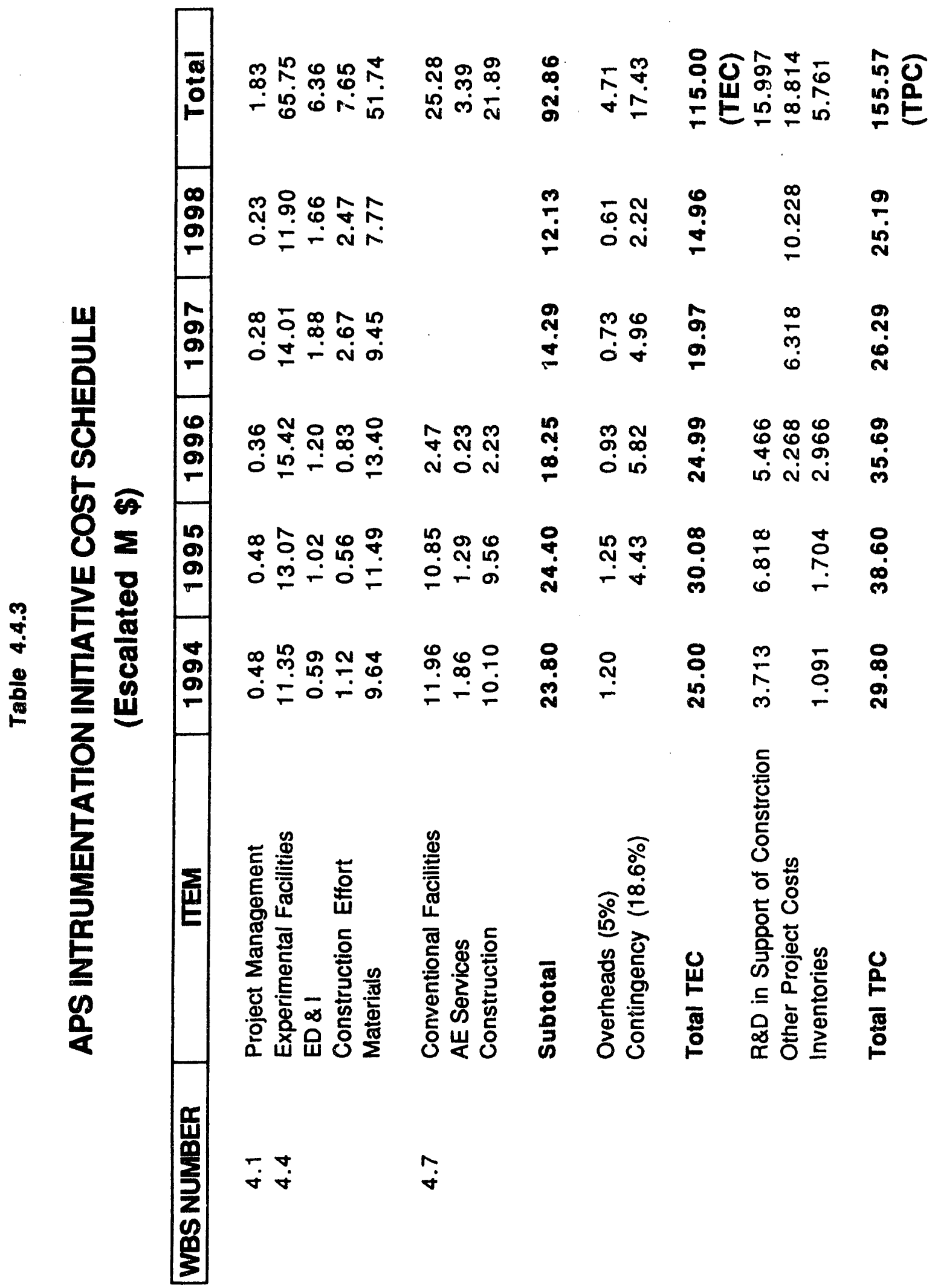




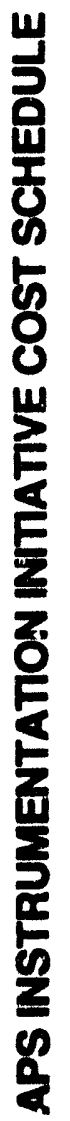

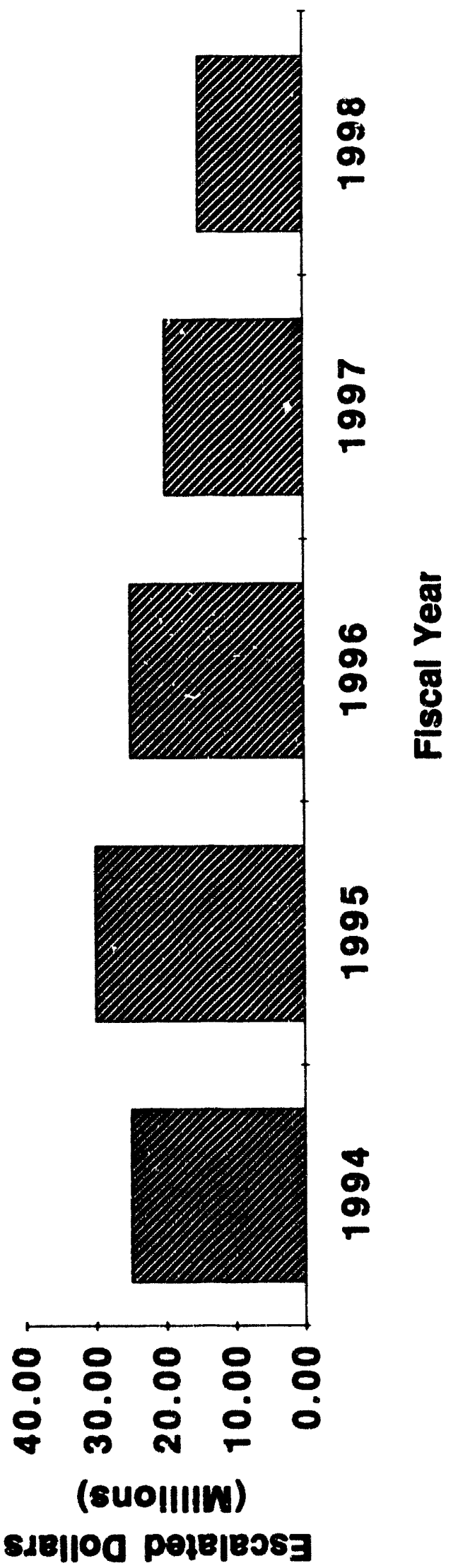

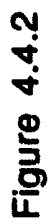




$$
\begin{aligned}
& \begin{array}{ll}
1 & 8 \\
6 & 0 \\
\hline & 5
\end{array}
\end{aligned}
$$

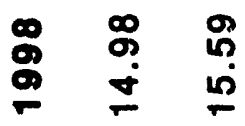

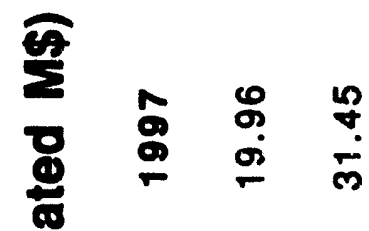

$$
\begin{aligned}
& \text { es }
\end{aligned}
$$

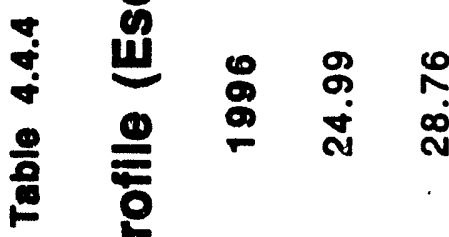

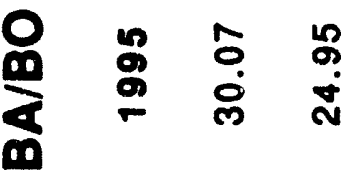

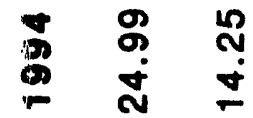

$$
\begin{aligned}
& \leftarrow 0 \\
& 88 \\
& \text { है } \\
& \text { i⿱ }
\end{aligned}
$$



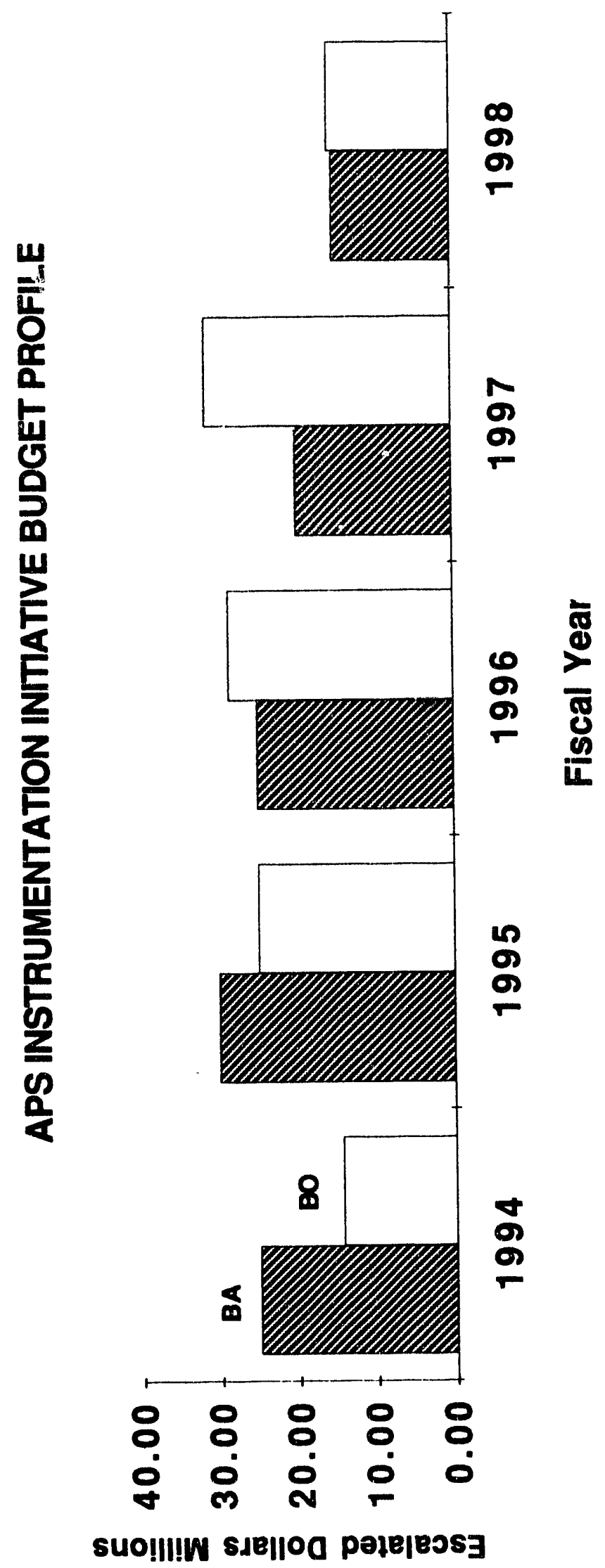


\section{APPENDIX A}

\section{PRECONSTRUCTION RESEARCh AND DEVELOPMENT PROGRAM}

\section{A.1 Introduction}

Assuming that the construction of APS Instrumentation Initiative will begin in FY 1994, the scope of the R\&D program has been described in this Appendix. It is clear that much emphasis of this R\&D will be in the instrumentation development. However, it should be realized that there are various elements of accelerator systems that will strongly interact with the requirements of the experimental facilities instrumentation.

Many of the components of the experimental facilities in the Instrumentation Initiative will be one of the kind. Hence, extra assurance must be established in the final performance of the facilities through a strong R\&D program on all the subcomponents.

Key R\&D items are scheduled with decision-making milestones that will be phased appropriately with the construction schedule of the new initiative.

\section{A.2 Insertion Devices for Type-B śectors}

The uniqueness of the IDs required for Type B Sectors in the Instrumentation Initiative demands an early beginning of PU. $J$ stuaies in the following areas:

- UHV requirements in the straight section in which in-vacuum IDs will be introduced.

- ID baking capabilities and influence on the magnetic performance.

- Capabilities to perform in-situ magnetic measurements on in-vacuum IDs.

- ID vacuum chamber prototype design for in-vacuum IDs, superconducting IDs, polarization IDs and multiple-source IDs.

- Influence of new IDs on the storage-ring performance.

- $R f$ interaction with in-vacuum IDs and unusual ID vacuum chamber geometries.

- Heat loads from the IDs proposed in the new initiative on the storage-ring vacuum chamber.

- Development of new magnet geometries for various IDs planned for the Instrumentation Initiative.

- R\&D to define new devices capable of delivering unique radiation properties. 


\section{A.3 Beamline Front Finds in Type B Sectors}

Anticipated heat loads that will have to be handled by the front end components on Type B Sectors demand enhancement of heat transfer capabilities by an order of magnitude. Such requirements can only be met by extensive engineering $R \& D$ in developing new heat transfer techniques and through the identification and testing of new materials for these unique applications.

The R\&D activities will address the needs of almost all the components of the front ends. The emphasis will be in the following areas:

- Beam stops.

- Apertures.

- Photon beam position monitors.

- Safety shutters.

- Windows for beamlines.

- Personnel safety and control.

- Detailed radiation safety analysis.

Because the requirements will widely vary from one front end to another in the Type B Sectors, early beginning of this activity is essential. These activities will use the experience and capabilities gained in developing the beamlines for the current construction project.

\section{A.4 Beamline Components in Type B Sectors}

This R\&D program can simply be divided into the following:

- Radiation physics

- X-ray optics

- Metrology

- Detector development

- Storage Ring Performance

The beamlines to be developed by the SRI CAT (primarily made up of APS Experimental Facilities staff) will provide an environment to perform much of the R\&D needed in the above areas. Again, requirements of Type B Sector beamlines are unique and differ from one beamline to the next. However, the R\&D 
requirements share some common needs. They include the $R \& D$ on unique radiation properties, high heat load optics, metrology capabilities, beamline controls, detector capabilities, and data acquisition, handling, and analysis. For each of the new IDs the performance of the operation of each of the storage ring components will have to be developed in detail, as will the dynamics of the positron lattice.

\section{A.5 Cost and Schedule for Preconstruction Research and Development Program}

The cost and schedule for accomplishing the preconstruction R\&D work in support of the Instrumentation Initaitive are based on the brief narrative of R\&D items identified in this appendix. The profile emphasizes the requirements of Type B Sectors described in Chapter II of this report. One of the most important considerations in developing the schedule is the need for a smooth transition from the current construction phase to the R\&D phase for the new initiative and from the R\&D for the new initiative to the Type B Sector construction phase for the new initative.

Costs for preconstruction R\&D effort, materials and services, and equipment are shown in Table A.1. The dollars shown are cost escalated in each fiscal year. The dollar figures shown here are consistent with the Schedule 44 document submitted to the DOE in December 1992. 
APPENDIX B

COPY OF SCHEDULE 44 


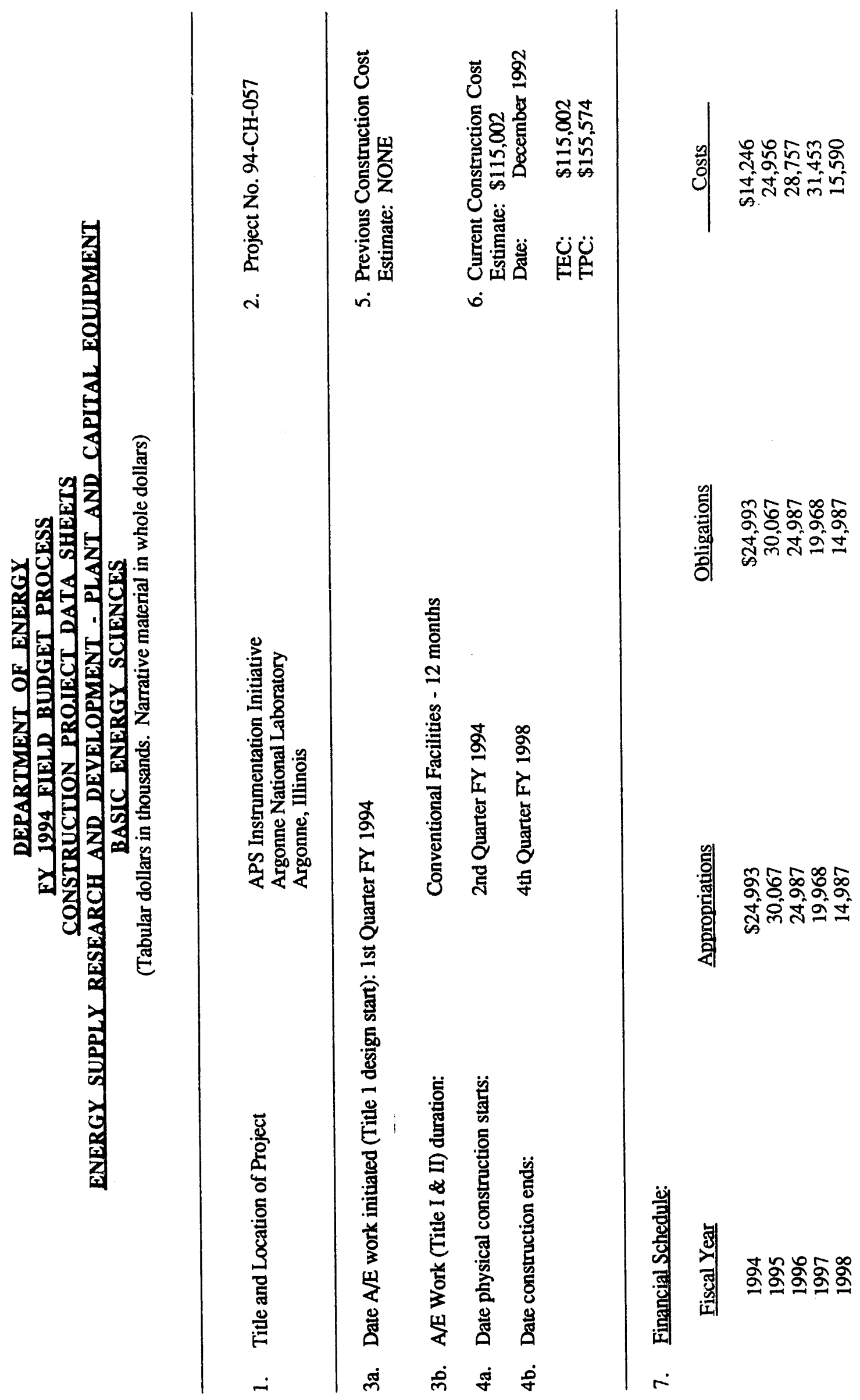




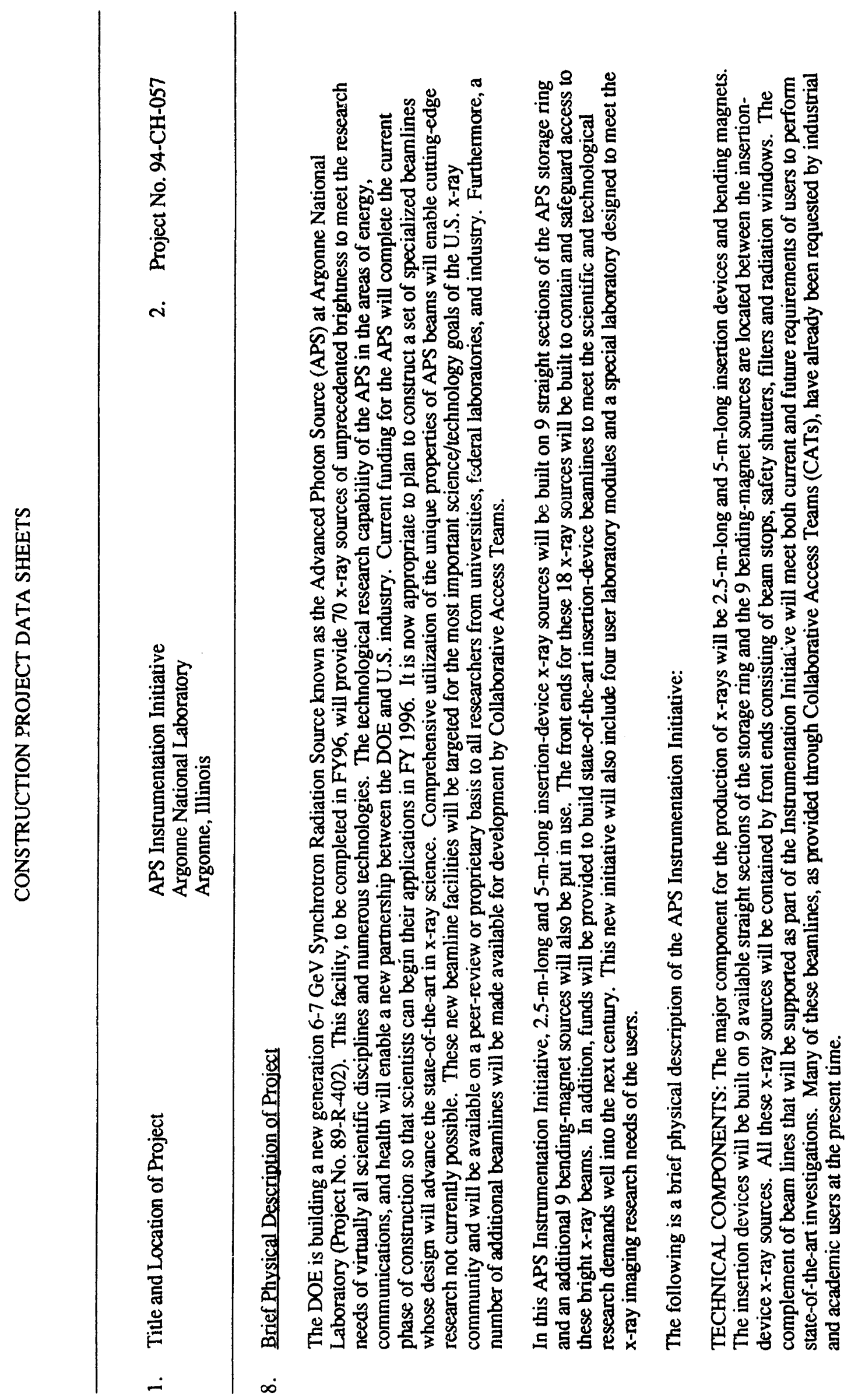


密兽密

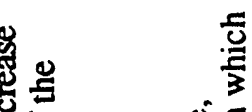
突要

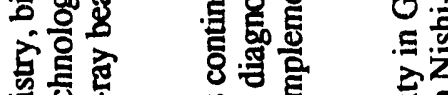

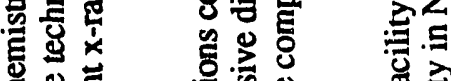

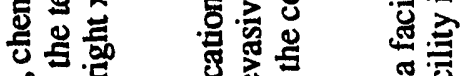

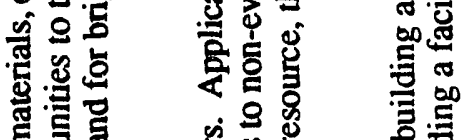

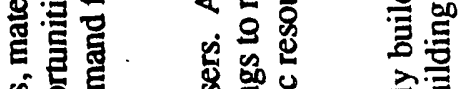

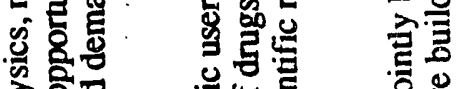

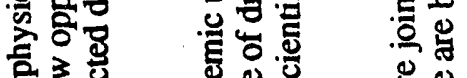

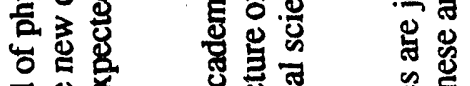

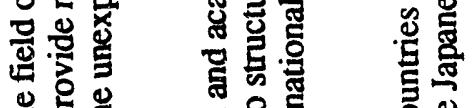

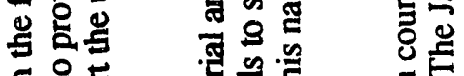

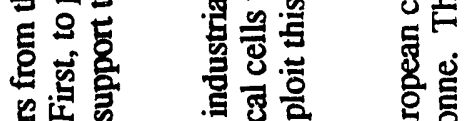

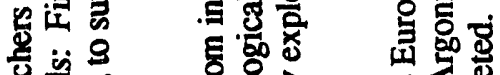

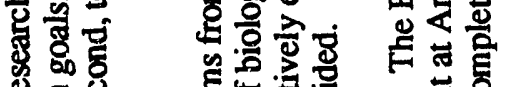
矛触

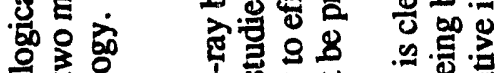
응 \& 要

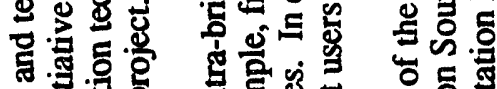

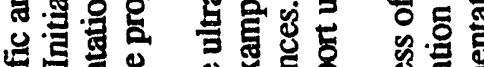

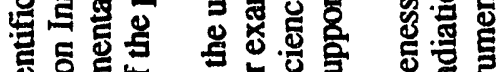

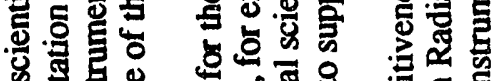

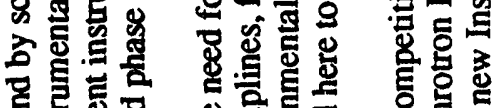

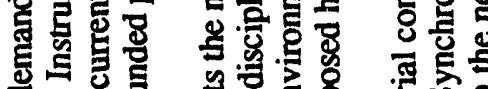
5 골 간

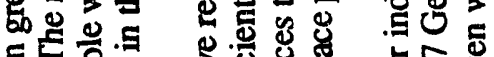
论

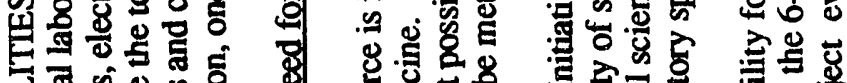




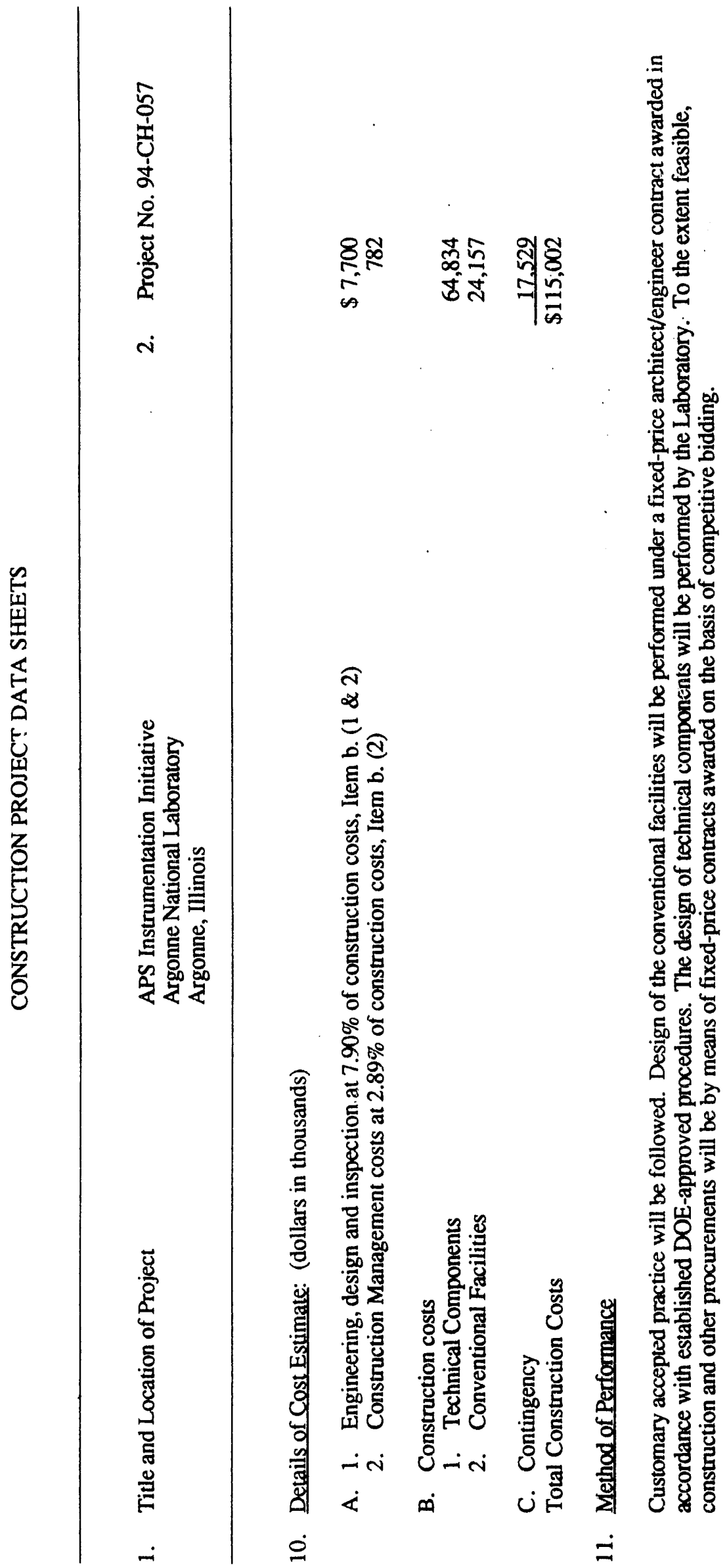




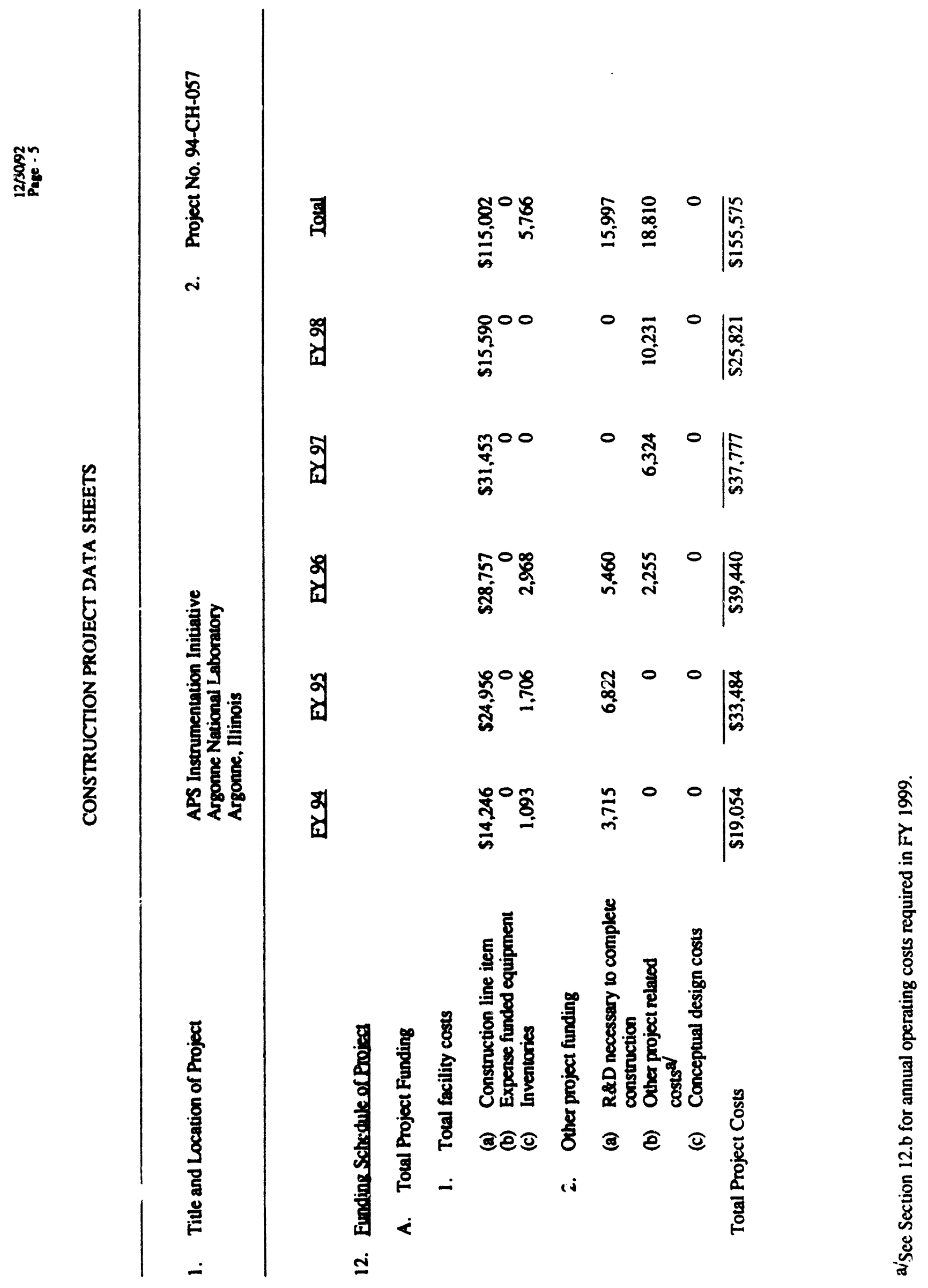



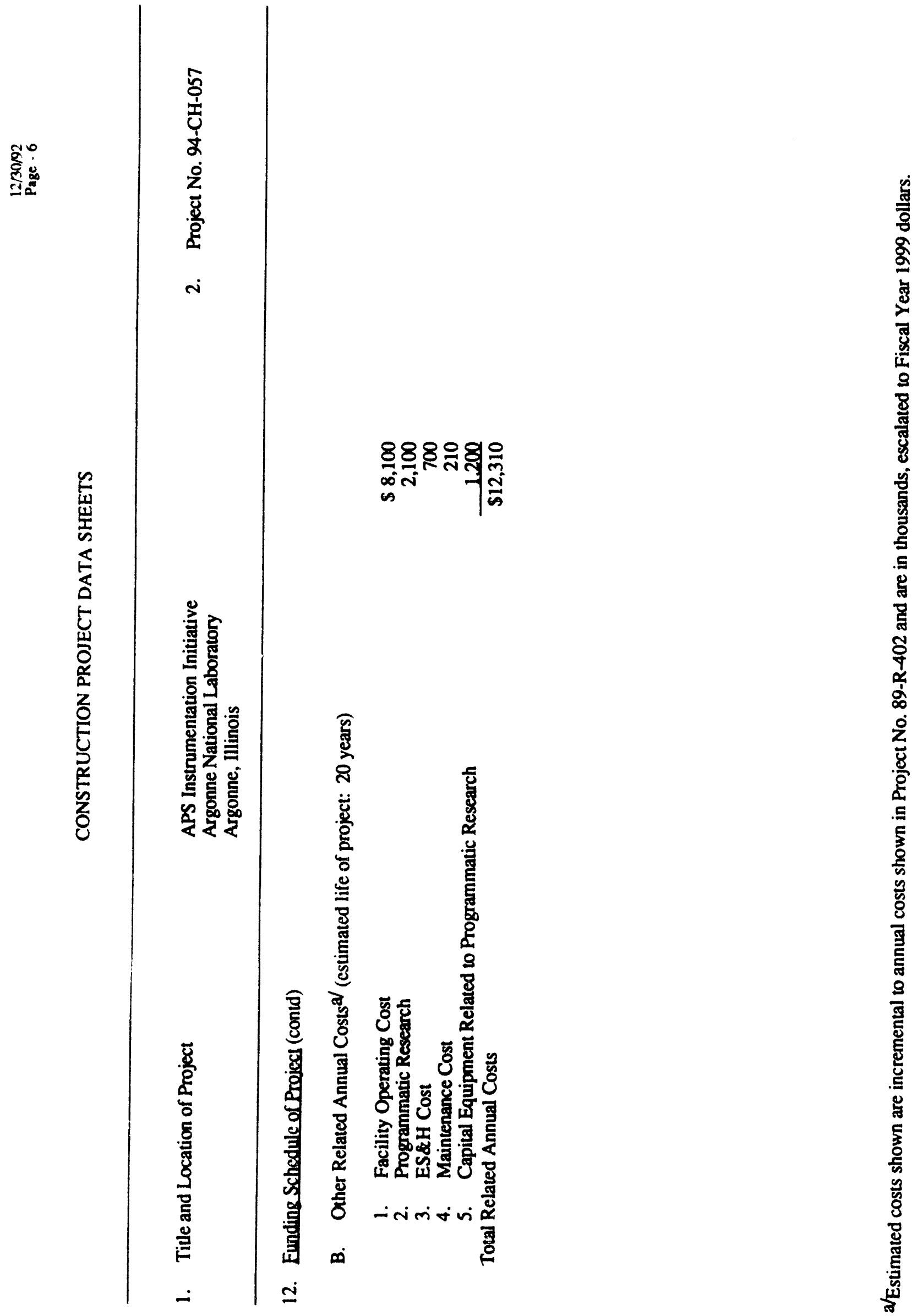


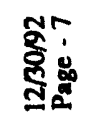

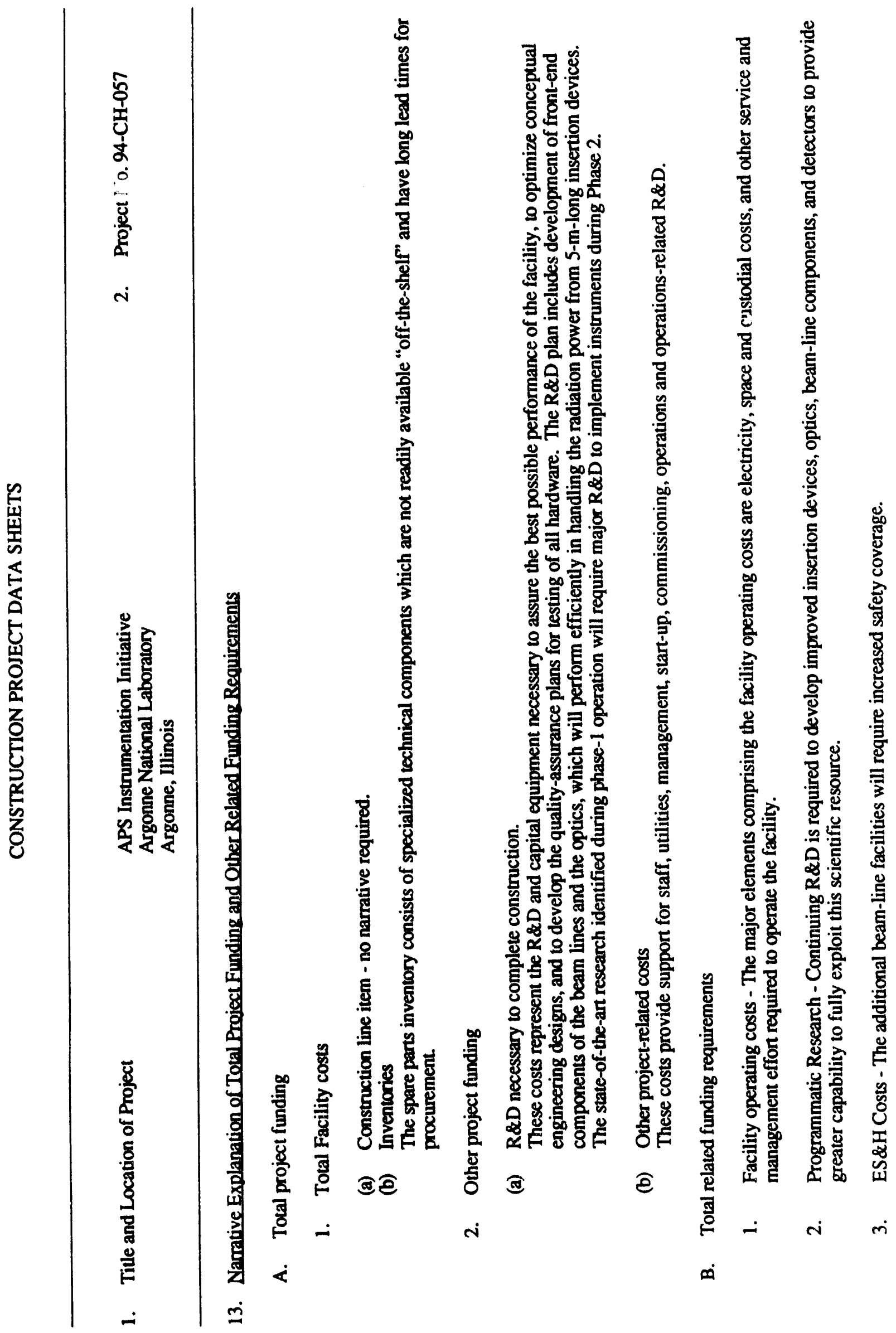




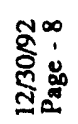

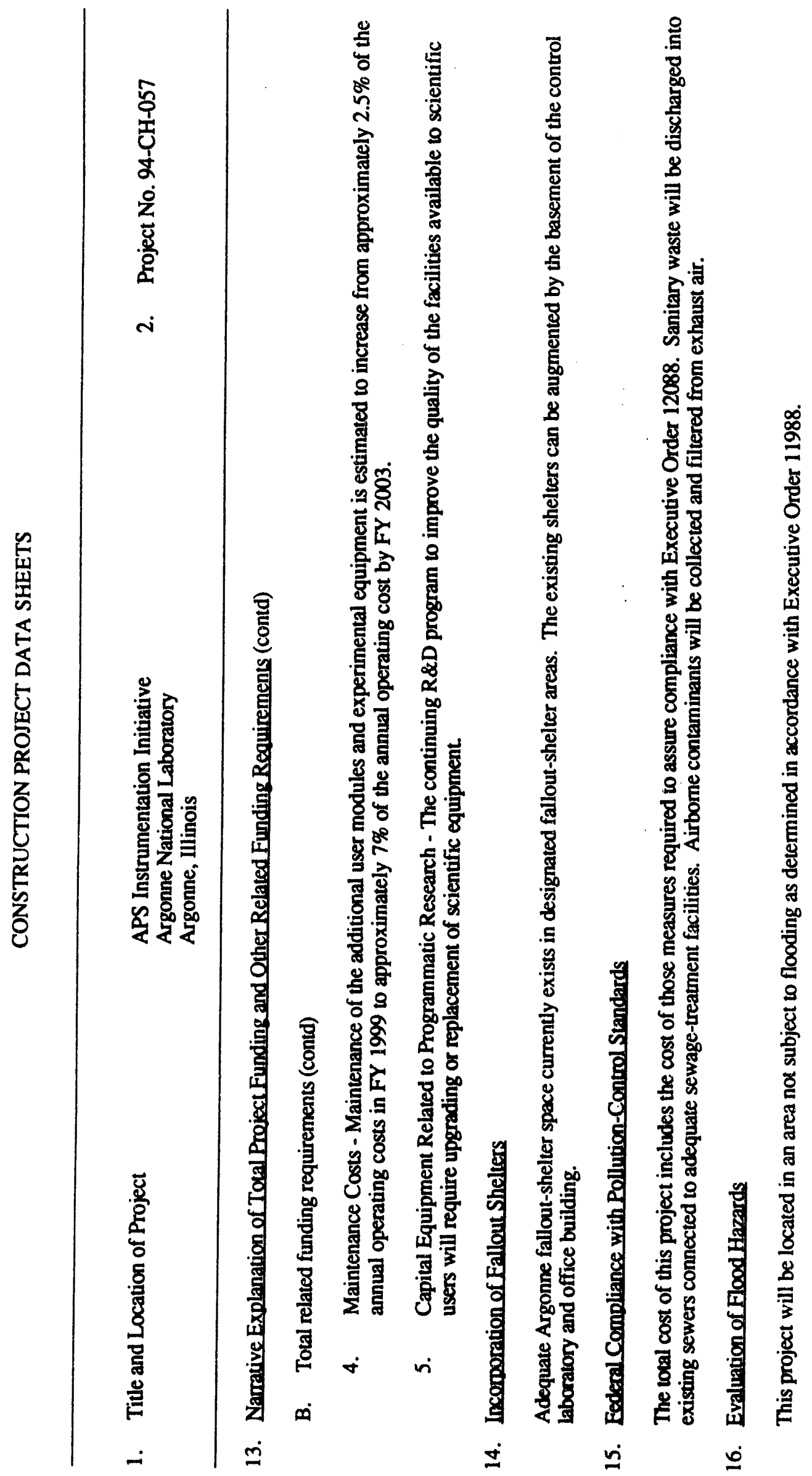




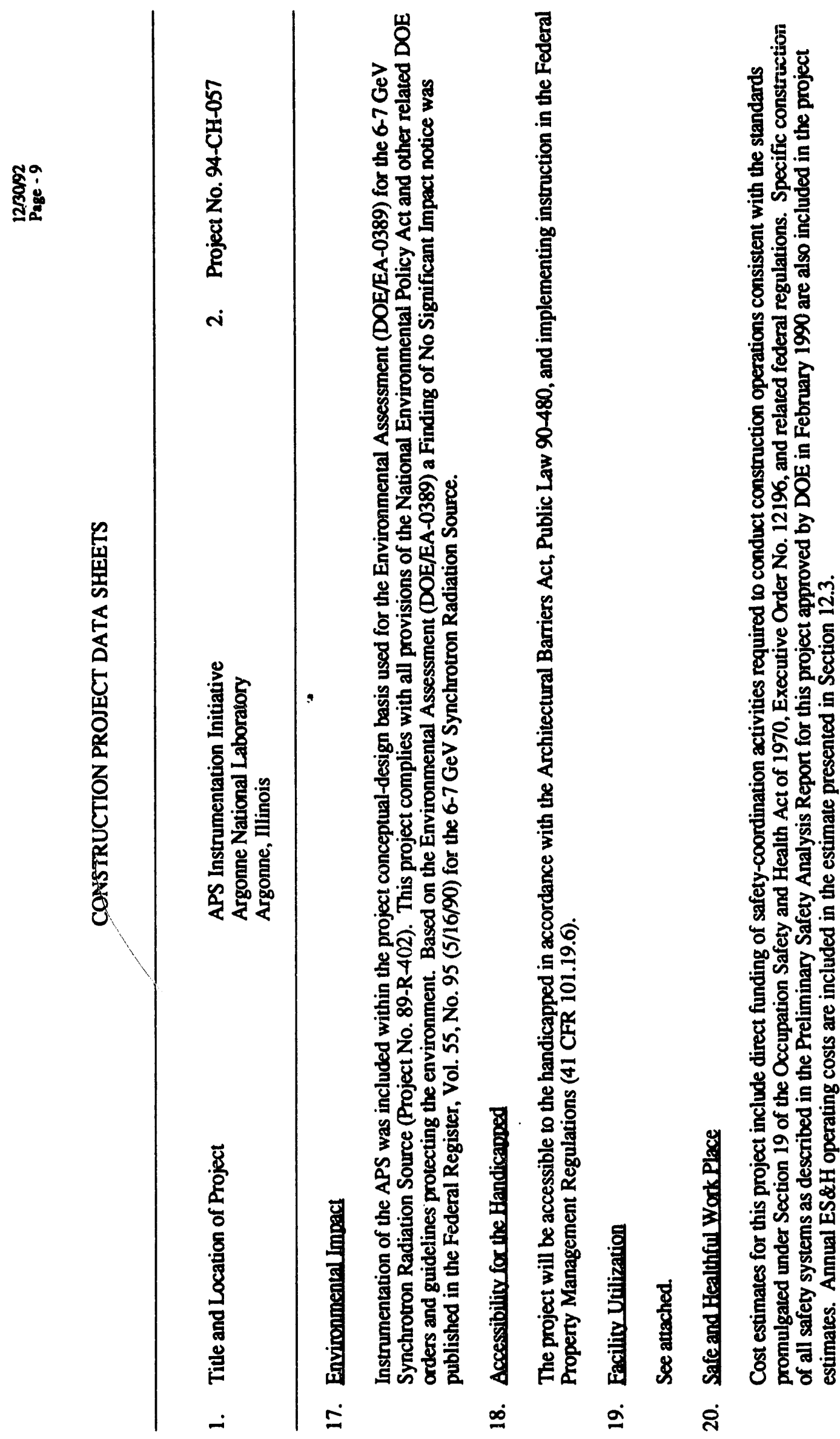




\section{Distribution for ANL-92/20}

Internal:
Y. Cho
J. Galayda
D. Getz
D. Moncton
G. Shenoy (103)

B. Sproule

E. Temple

APS Document Control Center (2) ANL Patent Dept. TIS Files

External:

DOE-OSTI, for distribution per UC-411 (34)

DOE Chicago Field Office:

Manager

ANL-E Libraries (3)

ANL-W Library

University of Chicago Board of Governors for Argonne National Laboratory, Committee to Advise the Laboratory Director on APS Construction (7) 

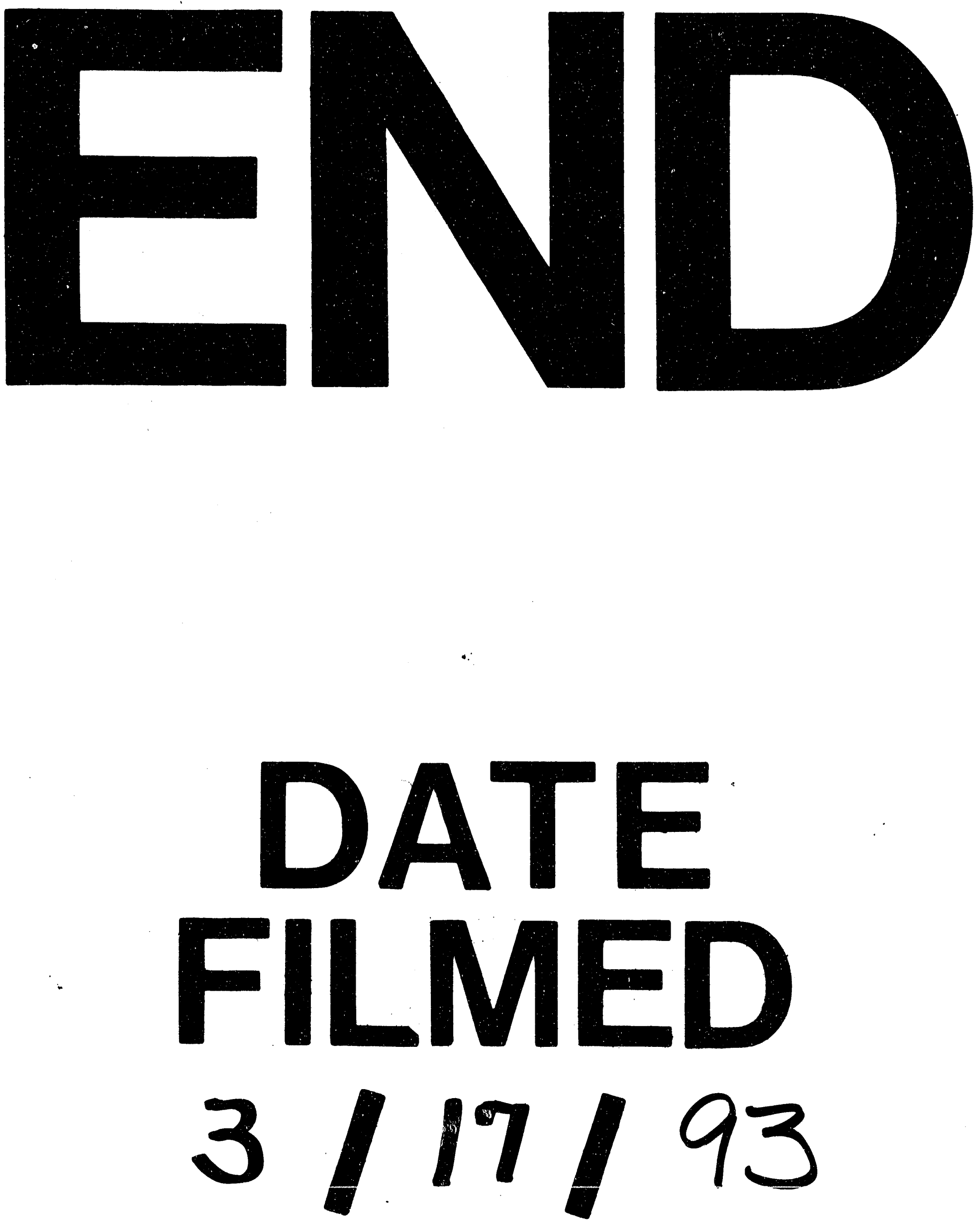
